\title{
Dose-response Modeling for Ordinal Outcome Data
}

\author{
by \\ Katrina Rogers-Stewart \\ A thesis submitted to the Faculty of Graduate and Postdoctoral Affairs \\ in partial fulfillment of the requirements for the degree of
}

Doctor of Philosophy

in

Probability and Statistics

School of Mathematics and Statistics

Ottawa-Carleton Institute for Mathematics and Statistics

Carleton University

Ottawa, Ontario

July 2015

(C) Copyright

Katrina Rogers-Stewart, 2015 


\section{Abstract}

We consider the characterization of a dose-response relationship when the response variable is ordinal in nature. Initially, we review the existing models for describing such a relationship, and propose an extension that allows for the possibility of non-zero probabilities of response for the different categories of the ordinal outcome variable associated with the control group. We illustrate via a simulation study the difficulties that can be encountered in model fitting when significant background responses are not acknowledged. In order to further enlarge the spectrum of dose-response relationships that can be accurately modeled, we introduce splines into the existing models for ordinal outcome data; demonstrating in a simulation that such models can provide a superior fit relative to existing ones. We also propose an alternative reference dose measure for ordinal responses. Specifically, we propose an alternative method for defining the benchmark dose, BMD, for ordinal outcome data. The approach yields an estimator that is robust to the number of ordinal categories into which we divide the response. In addition, the estimator is consistent with currently accepted definitions of the BMD for quantal and continuous data when the number of categories for the ordinal response is two, or become extremely large, respectively. We suggest two methods for determining an interval reflecting the lower confidence limit of the BMD; one based on the delta method, the other on a likelihood ratio approach. We 
show via a simulation study that intervals based on the latter approach are able to achieve the nominal level of coverage. 


\section{Acknowledgements}

I would like to acknowledge a number of individuals who helped make this work possible. Firstly, I would like to express my gratitude and appreciation to my supervisor Dr. Farrell and co-supervisor Dr. Nielsen for their guidance, assistance and support during my research and the completion of this thesis. I am also grateful to my parents and sister for their encouragement throughout my studies. Last but not

least, I would like to thank my husband, Mathieu, for his constant love, patience and understanding. 


\section{Contents}

$\begin{array}{lr}\text { Abstract } & \text { ii } \\ \text { List of Tables } & \text { viii } \\ \text { List of Figures } & \text { xiii }\end{array}$

Introduction $\quad 1$

1 Ordinal Models and a Background Parameter $\quad 4$

1.1 Introduction . . . . . . . . . . . . . . . . . . . 4

1.2 Ordinal Regression Models . . . . . . . . . . . . . . 6

1.2.1 Cumulative (CU) Link Models . . . . . . . . . . . . 8

1.2.2 Continuation-Ratio (CR) Link Models . . . . . . . . . . . . . 10

1.2.3 Adjacent Categories (AC) Link Models . . . . . . . . . . . . . 12

1.3 Maximum Likelihood Estimation . . . . . . . . . . . . 13

1.3.1 Cumulative (CU) Link Models . . . . . . . . . . . . . . 17

1.3.2 Continuation-Ratio (CR) Link Models . . . . . . . . . . . . 18

1.3.3 Adjacent Categories (AC) Link Models . . . . . . . . . . . . . 19

1.4 Background Response . . . . . . . . . . . . . . . . . 22 
1.4.1 Incorporating a Background Response With a Latent Process 22

1.4.2 Estimation of Background Model Parameters . . . . . . . . . 26

1.5 Simulation Study . . . . . . . . . . . . . . . . . . . 28

1.5.1 Glasgow Outcome Scale Data . . . . . . . . . . . . . . 30

1.5.2 Tinaroo Virus Data . . . . . . . . . . . . . . . . 35

1.6 Conclusion and Discussion . . . . . . . . . . . . . . . . . . . 39

2 Modelling Ordinal Data with Splines $\quad 41$

2.1 Introduction . . . . . . . . . . . . . . . . . . 41

2.2 Background . . . . . . . . . . . . . . . . . . . 42

2.2.1 Spline Smoothing . . . . . . . . . . . . . . . . 42

2.2.2 Monotonic Spline Smoothing . . . . . . . . . . . . . . 43

2.2.3 Ordinal Modeling and Estimation . . . . . . . . . . . . 44

2.2.4 Properties of Estimators . . . . . . . . . . . . . . . . . . . 49

2.2.5 Model Selection Criteria . . . . . . . . . . . . . 53

2.3 Monotone Smoothing Models for Ordinal Data . . . . . . . . . . . . 54

2.3.1 Estimation via Adaptive Fixed Knots . . . . . . . . . . . . . . 57

2.3.2 Estimation via Penalized Splines . . . . . . . . . . . 60

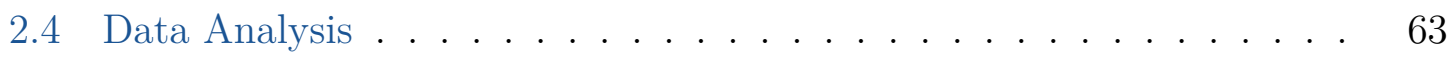

2.5 Simulation . . . . . . . . . . . . . . . . 67

2.5.1 Emulating a Dose-finding Study . . . . . . . . . . . . . . 67

2.5.2 Simulation Investigating Estimator Properties . . . . . . . . . 73

2.6 Conclusion . . . . . . . . . . . . . . . . . . . 85

3 The Benchmark Dose for Ordinal Models 86

3.1 Introduction . . . . . . . . . . . . . . 86 
3.2 Definitions of the Benchmark Dose . . . . . . . . . . 88

3.2 .1 Quantal Response . . . . . . . . . . . . . . . . . . . 89

3.2 .2 Continuous Response . . . . . . . . . . . . . . . . . . 90

3.2.3 Non-Quantal, Non-Continuous Responses . . . . . . . . . . . 93

3.2.4 Ordinal Response as Proposed by Chen and Chen . . . . . . . 95

3.3 Proposed Benchmark Dose for Ordinal Response . . . . . . . . . . . . 99

3.3 .1 Motivation . . . . . . . . . . . . . . . . . . . . 99

3.3 .2 Details . . . . . . . . . . . . . . . . . . . 100

3.4 Calculating the Lower Confidence Limit of the Benchmark Dose (BMDL) 104

3.4.1 Delta Method Using the Wald Statistic . . . . . . . . . . . 104

3.4.2 Likelihood Ratio Based Confidence Interval _ . . . . . . . 105

3.5 Simulation . . . . . . . . . . . . . . . . . . 107

3.5.1 Investigation of the OBMD Estimator . . . . . . . . . 107

3.5.2 Investigation of the Lower Confidence Limit of OBMD $\ldots \ldots 110$

3.6 Conclusion . . . . . . . . . . . . . . . . . . . . . . . . . . 115

$\begin{array}{ll}\text { Bibliography } & 116\end{array}$

$\begin{array}{lll}\text { Appendix A Simulation Results for Spline Models } & 123\end{array}$

Appendix B Benchmark Dose Simulation Results 154 


\section{List of Tables}

1.1 Responses of trauma patients on the Glasgow Outcome Scale. . . . . 28

1.2 Responses of chicken embryos exposed to the Tinaroo virus. . . . . . 29

1.3 AIC of CU, CR and AC fits to the Glasgow Outcome Scale data. . . 30

1.4 Mean AIC of CU, CR and AC fits for the Glasgow Outcome Scale simulation. . . . . . . . . . . . . . . . . . . 32

1.5 Mean residuals for the Glasgow Outcome Scale simulation. . . . . . . 34

1.6 Log-likelihood and AIC of CU fits to the Tinaroo virus data. . . . . . 36

1.7 Estimated response category probabilities from the CURB fit to the Tinaroo virus data. . . . . . . . . . . . . . . . . . . 36

1.8 Mean Log-likelihood and mean AIC for the simulation based on the Tinaroo virus data. . . . . . . . . . . . . . . . . 37

1.9 Mean residuals and standard deviations for the simulation based on the Tinaroo virus data. . . . . . . . . . . . . . . . . . . . 38

1.10 Mean estimates and sample standard deviations of the parameters for the CURB model for the simulation based on the Tinaroo virus data.

2.1 Incidences of selected histopathological lesions in rats exposed to dietary $1,1,2,2$-tetrachlorethane for 14 weeks. . . . . . . . . . . 
2.2 Summary of model properties and selection criteria for fits to the 1,1,2,2-tetrachlorethane data. . . . . . . . . . . . . . 64

2.3 Mean AIC of CUR and PMS fits for the simulations with data generated from the probabilities (2.27) and (2.29) . . . . . . . . . 69

3.1 Simulation results investigating OBMD across different values of $C$. . 108

3.2 Simulation results investigating estimators of the lower confidence limit of OBMD across different values of $C$ for three nested designs. . . . . 113

3.3 Simulation results investigating estimators of the lower confidence limit of OBMD for $C=4$ across various designs. . . . . . . . . . . . . . . 114

A.1 Summaries of $\hat{\boldsymbol{\theta}}$ for the Fixed Knot Model over 1000 Simulations . . . 124

A.2 Summaries of $\hat{\boldsymbol{\theta}}$ for the Fixed Knot Model over 1000 Simulations . . . 125

A.3 Summaries of $\hat{\boldsymbol{\theta}}$ for the Fixed Knot Model over 1000 Simulations . . . 126

A.4 Summaries of Standard Error Estimates of $\boldsymbol{\theta}$ for the Fixed Knot Model over 1000 Simulations . . . . . . . . . . . . . . . . . . . . . 127

A.5 Summaries of Standard Error Estimates of $\boldsymbol{\theta}$ for the Fixed Knot Model over 1000 Simulations . . . . . . . . . . . . . . . . . . 128

A.6 Summaries of Standard Error Estimates of $\boldsymbol{\theta}$ for the Fixed Knot Model over 1000 Simulations . . . . . . . . . . . . . . . . . . . 129

A.7 Summaries of $\Psi(z) \hat{\boldsymbol{\beta}}$ for Selected $x$ for the Fixed Knot Model over 1000 Simulations . . . . . . . . . . . . . . . . 130

A.8 Summaries of $\Psi(z) \hat{\boldsymbol{\beta}}$ for Selected $x$ for the Fixed Knot Model over

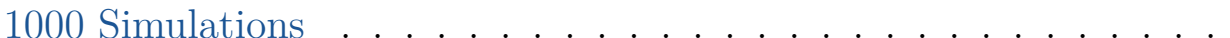

A.9 Summaries of $\Psi(z) \hat{\boldsymbol{\beta}}$ for Selected $x$ for the Fixed Knot Model over 1000 Simulations ... . . . . . . . . . . . . . . 132 
A.10 Summaries of $\hat{\boldsymbol{\eta}}(z)$ for Selected $x$ for the Fixed Knot Model over 1000

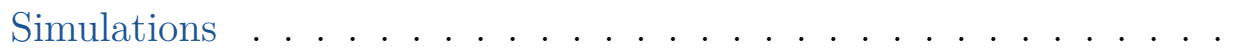

A.11 Summaries of $\hat{\boldsymbol{\eta}}(z)$ for Selected $x$ for the Fixed Knot Model over 1000



A.12 Summaries of $\hat{\boldsymbol{\eta}}(z)$ for Selected $x$ for the Fixed Knot Model over 1000

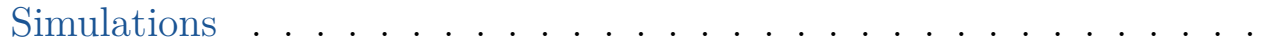

A.13 Summaries of $\sum_{k=1}^{j} \hat{\pi}_{k}(z)$ for Selected $x$ for the Fixed Knot Model over 1000 Simulations . . . . . . . . . . . . . . . .

A.14 Summaries of $\sum_{k=1}^{j} \hat{\pi}_{k}(z)$ for Selected $x$ for the Fixed Knot Model over 1000 Simulations . . . . . . . . . . . . . . . . . . 137

A.15 Summaries of $\sum_{k=1}^{j} \hat{\pi}_{k}(z)$ for Selected $x$ for the Fixed Knot Model over 1000 Simulations . . . . . . . . . . . . . . . .

A.16 Summaries of Selected $\hat{\boldsymbol{\theta}}$ for the Penalized Model Standard Errors Simulation with 1000 Simulations . . . . . . . . . . . .

A.17 Summaries of Selected $\hat{\boldsymbol{\theta}}$ for the Penalized Model Standard Errors Simulation with 1000 Simulations . . . . . . . . . . . . . . 140

A.18 Summaries of Selected $\hat{\boldsymbol{\theta}}$ for the Penalized Model Standard Errors Simulation with 1000 Simulations . . . . . . . . . . . . . . 141

A.19 Summaries of Standard Error Estimates of Selected $\boldsymbol{\theta}$ for the Penalized Model Standard Errors Simulation with 1000 Simulations . . . . . . . 142

A.20 Summaries of Standard Error Estimates of Selected $\boldsymbol{\theta}$ for the Penalized Model Standard Errors Simulation with 1000 Simulations . . . . . . .

A.21 Summaries of Standard Error Estimates of Selected $\boldsymbol{\theta}$ for the Penalized Model Standard Errors Simulation with 1000 Simulations . . . . . . . 144 
A.22 Summaries of $\boldsymbol{\Psi}(z) \hat{\boldsymbol{\beta}}$ for Selected $x$ for the Penalized Model Standard Errors Simulation with 1000 Simulations . . . . . . . . . . . . 145

A.23 Summaries of $\Psi(z) \hat{\boldsymbol{\beta}}$ for Selected $x$ for the Penalized Model Standard Errors Simulation with 1000 Simulations . . . . . . . . . . . . 146

A.24 Summaries of $\Psi(z) \hat{\boldsymbol{\beta}}$ for Selected $x$ for the Penalized Model Standard Errors Simulation with 1000 Simulations . . . . . . . . . . . . 147

A.25 Summaries of $\hat{\boldsymbol{\eta}}(z)$ for Selected $x$ for the Penalized Model Standard Errors Simulation with 1000 Simulations . . . . . . . . . . . . . . 148

A.26 Summaries of $\hat{\boldsymbol{\eta}}(z)$ for Selected $x$ for the Penalized Model Standard Errors Simulation with 1000 Simulations . . . . . . . . . . . . . 149

A.27 Summaries of $\hat{\boldsymbol{\eta}}(z)$ for Selected $x$ for the Penalized Model Standard Errors Simulation with 1000 Simulations . . . . . . . . . . . . 150

A.28 Summaries of $\sum_{k=1}^{j} \hat{\pi}_{k}(z)$ for Selected $x$ for the Penalized Model Standard Errors Simulation with 1000 Simulations . . . . . . . . . . . 151

A.29 Summaries of $\sum_{k=1}^{j} \hat{\pi}_{k}(z)$ for Selected $x$ for the Penalized Model Standard Errors Simulation with 1000 Simulations . . . . . . . . . . . . 152

A.30 Summaries of $\sum_{k=1}^{j} \hat{\pi}_{k}(z)$ for Selected $x$ for the Penalized Model Standard Errors Simulation with 1000 Simulations . . . . . . . . . . . 153

B.1 Simulation results investigating OBMD and estimators of the lower confidence limit across designs with various number of doses and repetitions per dose for $C=3$. The BMDLN and BMDLX estimators have




B.2 Simulation results investigating OBMD and estimators of the lower confidence limit across designs with various number of doses and repetitions per dose for $C=4$. The BMDLN and BMDLX estimators have a nominal confidence level of $95 \%$. . . . . . . . . . . . . . 156

B.3 Simulation results investigating OBMD and estimators of the lower confidence limit across designs with various number of doses and repetitions per dose for $C=5$. The BMDLN and BMDLX estimators have a nominal confidence level of $95 \%$. . . . . . . . . . . . . . . . 157 


\section{List of Figures}

1.1 Probability curves for reduced and full models from each of the cumulative, continuation-ratio and adjacent categories families. . . . . . . 11

1.2 Probability curves for CURB with various $\tau \ldots \ldots \ldots \ldots \ldots$

1.3 Cumulative probability estimates of $\mathrm{CU}, \mathrm{CR}$ and $\mathrm{AC}$ fits to the Glasgow Outcome Scale data. . . . . . . . . . . . . . . . . . 31

1.4 Mean cumulative probability estimates of CU, CR and AC fits for the Glasgow Outcome Scale simulation. . . . . . . . . . . . . . 33

1.5 Cumulative probability estimates of $\mathrm{CU}$ fits to the Tinaroo virus data. 35

1.6 Mean cumulative probability estimates of $\mathrm{CU}$ fits for the simulation based on Tinaroo virus data. . . . . . . . . . . . . . . . . . 37

2.1 Cumulative probability estimates of CUR, AMS and PMS fits to the 1,1,2,2-tetrachlorethane data. . . . . . . . . . . . . .

2.2 Cumulative probability estimates of AS, AMS, PS and PMS fits to the $1,1,2,2$-tetrachlorethane data. . . . . . . . . . . . . 66

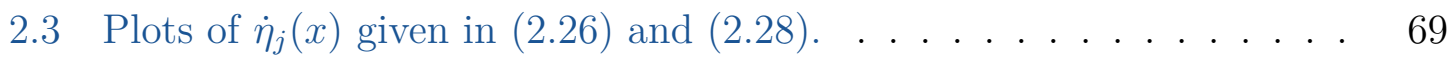

2.4 Estimated bias of $\hat{\eta}_{j}(x)$ of CUR and PMS fits for the simulations with data generated from the probabilities (2.27) and (2.29). . . . 70 
2.5 Estimated coverage of $\hat{\eta}_{j}(x)$ of CUR and PMS fits for the simulations with data generated from the probabilities (2.27) and (2.29). . . . . 71

2.6 Plot of the true $\eta_{j}(x)$ used in the FXMS simulation. . . . . . . . . 76

2.7 Estimated bias of $\hat{\eta}_{j}(x), j=1,2$, over 1,000 simulated replicates for the FXMS model and each of nine designs. . . . . . . . . . . . . . . 77

2.8 Mean model-based and Jackknife standard errors of $\hat{\eta}_{j}(x), j=1,2$, over 1,000 simulated replicates for the FXMS model and each of nine designs. The Monte Carlo standard error estimate is also displayed.

2.9 Model-based and Jackknife coverage rates of $95 \%$ confidence intervals for $\eta_{j}(x), j=1,2$, over 1,000 simulated replicates for the FXMS model and each of nine designs. . . . . . . . . . . . . . . . . . . . . 79

2.10 Plot of the true $\eta_{j}(x)$ used in the PMS simulation. . . . . . . . . . . 81

2.11 Estimated bias of $\hat{\eta}_{j}(x), j=1,2$, over 1,000 simulated replicates for the PMS model and each of nine designs. . . . . . . . . . . . .

2.12 Mean model-based and Jackknife standard errors of $\hat{\eta}_{j}(x), j=1,2$, over 1,000 simulated replicates for the PMS model and each of nine designs. The Monte Carlo standard error estimate is also displayed. .

2.13 Model-based and Jackknife coverage rates of 95\% confidence intervals for $\eta_{j}(x), j=1,2$, over 1,000 simulated replicates for the PMS model and each of nine designs. . . . . . . . . . . . . . . . . 84

3.1 Dichotomizing a continuous variable to a quantal variable. . . . . . . 94

3.2 Categorizing a continuous variable to an ordinal variable. . . . . . . . 96

3.3 OBMD and CCBMD values where the true distribution follows a probit CUR model. . . . . . . . . . . . . . . . . . . 


\section{Introduction}

\section{Dose-response Modeling for Ordinal Outcome Data}

Pre-market testing of new drugs often involves the characterization of a dose-response relationship. To develop such a relationship, subjects are randomly assigned to a control (placebo) and various dose level groups for the drug under consideration. While there is a vast array of research that has relied on the characterization of dose-response relationships, the majority has focused on responses that are binary in nature. In order to allow for the possibility of a non-zero probability of response for the control group, such studies centring on Bernoulli outcomes have relied on the Hill model to describe the dose-response relationship. The Hill model can be viewed as an extension to the standard single-covariate logistic regression model. Specifically, a background parameter is incorporated into the latter to allow for the possibility that the probability of a response for the control group is not zero.

As indicated above, much research into the study of dose-response relationships has been devoted to binary responses. By contrast, relatively little work has considered ordinal outcomes where there are more than two possible responses for each 
subject, and these responses possess a natural ordering. In this thesis, we shall consider dose-response models for ordinal outcome data.

In Chapter 1, we begin by presenting a review of the existing models for such responses. We subsequently present extensions to the standard ordinal models to include a background response vector, which allows for the possibility of non-zero probabilities of response for the different categories of the ordinal outcome variable associated with the control group. We illustrate via a simulation study the difficulties that can be encountered in model fitting when significant background responses are not acknowledged.

We remark at the end of Chapter 1 that, despite the findings observed, there will be dose response curves for ordinal outcome data that, as a result of their shape, simply cannot be described well by the existing models, either with or without an acknowledgement of background response. In Chapter 2, we introduce monotone splines into the cumulative link ordinal model, and demonstrate that in some circumstances, such a model can provide a superior fit relative to the existing ones. We discuss two methods of estimation for the model; one based on fixed knots, the other on penalized splines. We show that the latter provides greater flexibility. We also propose two useful estimates of standard error for the estimators of the model parameters. One is based on the jackknife, the other on a model-based approach.

In Chapter 3, we turn our attention to the development of a reference dose measure for ordinal outcome data. Specifically, we propose an alternative method for defining the benchmark dose, BMD, for ordinal outcome data. The approach yields an estimator that is robust to the number of ordinal categories into which we divide the response. In addition, the estimator is consistent with currently accepted definitions of the BMD for quantal and continuous data when the number of categories for 
the ordinal response is two, or become extremely large, respectively. We also suggest two methods for determining an interval reflecting the lower confidence limit of the BMD; one based on the delta method, the other on a likelihood ratio approach. We show via a simulation study that intervals based on the latter approach are able to achieve the nominal level of coverage. 


\section{Chapter 1}

\section{Ordinal Models and a Background Parameter}

\section{$1.1 \quad$ Introduction}

Pre-market testing of new drugs often involves the characterization of a dose-response relationship. To develop such a relationship, subjects are randomly assigned to a control (placebo) and various dose level groups for the drug under consideration. We consider parallel-group designs here, in which each subject receives only one dose level throughout the study. The response to be characterized can represent either the success of a treatment or a side-effect.

While there is a vast array of research that has relied on the characterization of dose-response relationships, the majority has focused on responses that are quantal or continuous in nature. In order to allow for the possibility of a non-zero probability of response for the control group, such studies centring on Bernoulli outcomes have relied on the Hill model (Hill, 1910) to describe the dose-response relationship. The Hill model can be viewed as an extension to the standard single-covariate logistic 
regression model. Specifically, a background parameter is incorporated into the latter to allow for the possibility that the probability of a response for the control group is not zero.

As indicated above, much research into the study of dose-response relationships has been devoted to quantal responses. By contrast, relatively little work has considered ordinal outcomes where there are more than two possible responses for each subject, and these responses possess a natural ordering. In this chapter, we shall consider dose-response models for ordinal outcome data. Initially, in Section 1.2, we present a review of the existing models for such responses. Each of these models can be assumed to possess separate or shared effects for the different categories of the outcome variable. Estimation of the parameters in these models is discussed in Section 1.3. In Section 1.4, we present a modeling framework derived from Xie and Simpson (1999) that extends any ordinal model to include a background response vector, which allows for the possibility of non-zero probabilities of response for the control dose and the different categories of the ordinal outcome variable. Section 1.4 also describes the procedure for estimation of these models with a background response. In Section 1.5, we initially fit the models discussed in Sections 1.2 and 1.4 to two data examples. The latter example illustrates the benefits that can be gained in model fit when a significantly large background response is acknowledged, rather than ignored. Motivated by these examples, we also present the results of a number of simulation studies aimed at investigating the properties of the estimators of the model parameters under a variety of different hypothesized scenarios. Conclusions and discussion are given in Section 1.6. 


\subsection{Ordinal Regression Models}

A random variable that can fall into $C$ categories is a categorical random variable; furthermore, if those categories are ordered in some manner then we can refer to the variable as ordinal. Suppose we have a $C$-category ordinal random variable, $Y$, and additionally that we have a vector $\mathbf{x}$ of $r$ explanatory variables, then $\pi_{j}(\mathbf{x})=P(Y=j \mid \mathbf{x})$ is the probability that category $j$ is observed and $\boldsymbol{\pi}(\mathbf{x})=\left(\pi_{1}(\mathbf{x}), \ldots, \pi_{C}(\mathbf{x})\right)^{\top}$ is the vector of response probabilities. Since $\sum_{j=1}^{C} \pi_{j}(\mathbf{x})=1$ we have that $Y$ has a multinomial probability distribution with parameter $\boldsymbol{\pi}(\mathbf{x})$.

In this thesis we concern ourselves with ordinal response variables with a focus on dose-response models. In such instances it is often the case that the last category reflects the strongest or most severe effect on a subject and that the first category indicates no (or minimal) effect, or apparent effect, on the subject. Also, in the case of clinical trials, the lowest level of dose is often a placebo, or one where a subject has not been exposed to a harmful substance; this group of individuals is referred to as the control group. In the dose-response context, one of the explanatory variables is a dosage level, or some transformation thereof, such as the log of the dosage.

In this chapter, we investigate the ordinal response models that are commonly referred to as cumulative link, continuation-ratio link and adjacent categories link models. Each of these models can be written in the form

$$
g\left(\gamma_{j}(\mathbf{x})\right)=\eta_{j}(\mathbf{x}), \quad j=1, \ldots, C-1
$$

where $g$ is some monotonic link function, $\gamma_{j}(\mathbf{x})$ a probability function involving $Y$ and $\eta_{j}(\mathbf{x})$ a function of the predictor $\mathbf{x}$. 
The form of the probabilities $\gamma_{j}(\mathbf{x})$ determines the relationship between the response variable $Y$ and $\eta_{j}(\mathbf{x})$; for the cumulative family $\gamma_{j}(\mathbf{x})=P(Y \leq j \mid \mathbf{x})$, the (forward) continuation-ratio family $\gamma_{j}(\mathbf{x})=P(Y=j \mid Y \geq j, \mathbf{x})$ and lastly for the adjacent categories family, $\gamma_{j}(\mathbf{x})=P(Y=j \mid Y=j$ or $Y=j+1, \mathbf{x})$. The backward continuation-ratio family, where $\gamma_{j}(\mathbf{x})=P(Y=j \mid Y \leq j, \mathbf{x})$, is another common family, however we do not consider it further. Note that the $C-1$ values of the vector $\gamma(\mathbf{x})=\left(\gamma_{1}(\mathbf{x}), \ldots, \gamma_{i, C-1}(\mathbf{x})\right)^{\top}$ fully determine the $C$ elements of $\boldsymbol{\pi}(\mathbf{x})$ since the elements of this latter vector are constrained to sum to 1 .

In this chapter we shall focus on the case where $\eta_{j}(\mathbf{x})$ is linear in the parameters. Specifically, we are interested in models of the form

$$
g\left(\gamma_{j}(\mathbf{x})\right)=\alpha_{j}+\boldsymbol{\beta}_{j}^{\top} \mathbf{x}, \quad j=1, \ldots, C-1
$$

with intercepts $\alpha_{j}$ and covariate effects $\boldsymbol{\beta}_{j}=\left(\beta_{j 1}, \ldots, \beta_{j r}\right)^{\top}$.

In some instances we may wish to collapse the $C-1$ covariate effects into a single common effect, $\boldsymbol{\beta}$. In this case the model in (1.2) becomes

$$
g\left(\gamma_{j}(\mathbf{x})\right)=\alpha_{j}+\boldsymbol{\beta}^{\top} \mathbf{x}, \quad j=1, \ldots, C-1
$$

where $\boldsymbol{\beta}=\left(\beta_{1}, \ldots, \beta_{r}\right)^{\top}$ is a vector of length $r$. This setup is more parsimonious requiring only $C-1+r$ parameters compared to the $(C-1)(r+1)$ parameters necessitated by (1.2). In the following sections we shall refer to (1.2) as a full model (F) and (1.3) as a reduced model (R). Also, if we let $\boldsymbol{\alpha}=\left(\alpha_{1}, \ldots, \alpha_{C-1}\right)^{\top}$, then we can write the parameter vector for the full model as $\boldsymbol{\theta}^{[F]}=\left(\boldsymbol{\alpha}^{\top}, \boldsymbol{\beta}_{1}^{\top}, \ldots, \boldsymbol{\beta}_{C-1}^{\top}\right)^{\top}$ and for the reduced model as $\boldsymbol{\theta}^{[R]}=\left(\boldsymbol{\alpha}^{\top}, \boldsymbol{\beta}^{\top}\right)^{\top}$.

In the two-category case, $C=2$, models (1.2) and (1.3) are generalized linear 
models (GLMs) (Nelder and Wedderburn, 1972), whereas if $C>2$ the response is a vector and so (1.2) and (1.3) are multivariate GLM, or in the terminology of Yee and Wild (1996) and Yee (2015), vector generalized linear models (VGLMs). In both GLMs and multivariate GLMs, the link function is a monotone increasing function with domain $[0,1]$. Some link functions commonly used in ordinal models include: the logit link, $g(p)=\log \left(\frac{p}{1-p}\right)$; the probit link, $g(p)=\Phi^{-1}$ where $\Phi^{-1}$ is the standard normal cumulative distribution function; the complementary log-log link, $g(p)=$ $\log \{-\log (1-p)\}$; and the log-log link, $g(p)=\log \{-\log (p)\}$. The canonical link for a multinomial is the logit and consequently this link is often used in practice.

In the remainder of this section we elaborate on using a specific family in the models (1.2) and (1.3). We consider three families, namely the cumulative, continuationratio and adjacent categories families in Sections 1.2.1, 1.2.2 and 1.2.3 respectively. For a general overview of these ordinal models see Chapters 3 and 4 of Agresti (2010) and Chapter 9 of Tutz (2011). Alternatively, Ananth and Kleinbaum (1997) and Regan and Catalano (2002) present a review with an epidemiological focus.

\subsubsection{Cumulative (CU) Link Models}

In this section we detail models (1.2) and (1.3) using the cumulative (CU) family.

Recall that the cumulative family has $\gamma_{j}(\mathbf{x})=P(Y \leq j \mid \mathbf{x})$, so the model (1.2) has the form

$$
g[P(Y \leq j \mid \mathbf{x})]=\alpha_{j}+\boldsymbol{\beta}_{j}^{\top} \mathbf{x}, \quad j=1, \ldots, C-1
$$

for some link function $g$. We refer to this model as the full cumulative link model, or CUF. It is important to note that without some restrictions on the parameter 
values it is possible to obtain invalid probabilities at some values of the parameter vector $\boldsymbol{\theta}$. To guarantee that the cumulative links are ordered, and to ensure that the probabilities are valid, we require the restriction that $\alpha_{j}+\boldsymbol{\beta}_{j}^{\top} \mathbf{x}<\alpha_{j+1}+\boldsymbol{\beta}_{j+1}^{\top} \mathbf{x}$, for $j=1, \ldots, C-1$ and all $\mathbf{x}$ in the domain of interest. The domain of interest of $\mathbf{x}$ must at least encompass any realized values $\mathbf{x}$, but could be larger. Such a restriction limits inference to $\mathbf{x}$ within the specified domain, and parameter estimates may vary depending upon the domain.

Alternatively, we can consider the model (1.3) which has a single shared effect,

$$
g[P(Y \leq j \mid \mathbf{x})]=\alpha_{j}+\boldsymbol{\beta}^{\top} \mathbf{x}, \quad j=1, \ldots, C-1
$$

and we refer to this reduced model as CUR. We again need to impose a restriction on the parameter values of $\boldsymbol{\theta}$, namely that the elements of $\boldsymbol{\alpha}=\left(\alpha_{1}, \ldots, \alpha_{C-1}\right)^{\top}$ be ordered, $\alpha_{1}<\alpha_{2}<\cdots<\alpha_{C-1}$. McCullagh (1980) proposed this model with a logit link and referred to it as the proportional odds model due to the special relationship between the odds of $P(Y \leq j \mid \mathbf{x})$ at $\mathbf{x}=\mathbf{x}^{[1]}$ and $\mathbf{x}=\mathbf{x}^{[2]}$. Specifically,

$$
\begin{aligned}
& \log \left(\frac{P\left(Y \leq j \mid \mathbf{x}^{[1]}\right) / P\left(Y>j \mid \mathbf{x}^{[1]}\right)}{P\left(Y \leq j \mid \mathbf{x}^{[2]}\right) / P\left(Y>j \mid \mathbf{x}^{[2]}\right)}\right) \\
& =\log \left(\frac{P\left(Y \leq j \mid \mathbf{x}^{[1]}\right)}{1-P\left(Y \leq j \mid \mathbf{x}^{[1]}\right)}\right)-\log \left(\frac{P\left(Y \leq j \mid \mathbf{x}^{[2]}\right)}{1-P\left(Y \leq j \mid \mathbf{x}^{[2]}\right)}\right) \\
& =g\left[P\left(Y \leq j \mid \mathbf{x}^{[1]}\right)\right]-g\left[P\left(Y \leq j \mid \mathbf{x}^{[2]}\right)\right] \\
& =\boldsymbol{\beta}^{\top}\left(\mathbf{x}^{[1]}-\mathbf{x}^{[2]}\right)
\end{aligned}
$$

and so the $\log$ of this ratio of odds is proportional to the distance between $\mathbf{x}^{[1]}$ and $\mathbf{x}^{[2]}$. Moreover, all the $C-1$ cumulative links result in the same quantity. This model 
is also known as the ordered link model.

The CUR has $C-1+r$ parameters and is more parsimonious than the CUF, but it only allows for cumulative probabilities curves from the link family which have a common slope. A property of these curves is that they approach either 0 or 1 as the covariates approach positive or negative infinity. On the other hand, the CUF has $(C-1)(r+1)$ parameters, and these extra parameters allow for more flexibility in the fit; specifically each of the cumulative probabilities curves may differ in slope. However, these curves must still approach either 0 or 1 as the covariates approach positive or negative infinity. The top panel of Figure 1.1 gives an example of cumulative probabilities curves that can be obtained with each of these two models.

\subsubsection{Continuation-Ratio (CR) Link Models}

The continuation-ratio (CR) family is defined by $\gamma_{j}(\mathbf{x})=P(Y=j \mid Y \geq j$, $\mathbf{x})$, and is particularly suited to situations where a subject passes through each category in order. An example in epidemiology is the progressive stages of an irreversible disease. Using the continuation-ratio family in model (1.2) gives

$$
g[P(Y=j \mid Y \geq j, \mathbf{x})]=\alpha_{j}+\boldsymbol{\beta}_{j}^{\top} \mathbf{x}, \quad j=1, \ldots, C-1,
$$

and we refer to this model as CRF. Unlike the corresponding model with the cumulative family (CUF in (1.4)), this model yields valid probabilities for all values of $\theta$.

The shared effect model (1.3) with the continuation-ratio family has the form

$$
g[P(Y=j \mid Y \geq j, \mathbf{x})]=\alpha_{j}+\boldsymbol{\beta}^{\boldsymbol{\top}} \mathbf{x}, \quad j=1, \ldots, C-1 .
$$




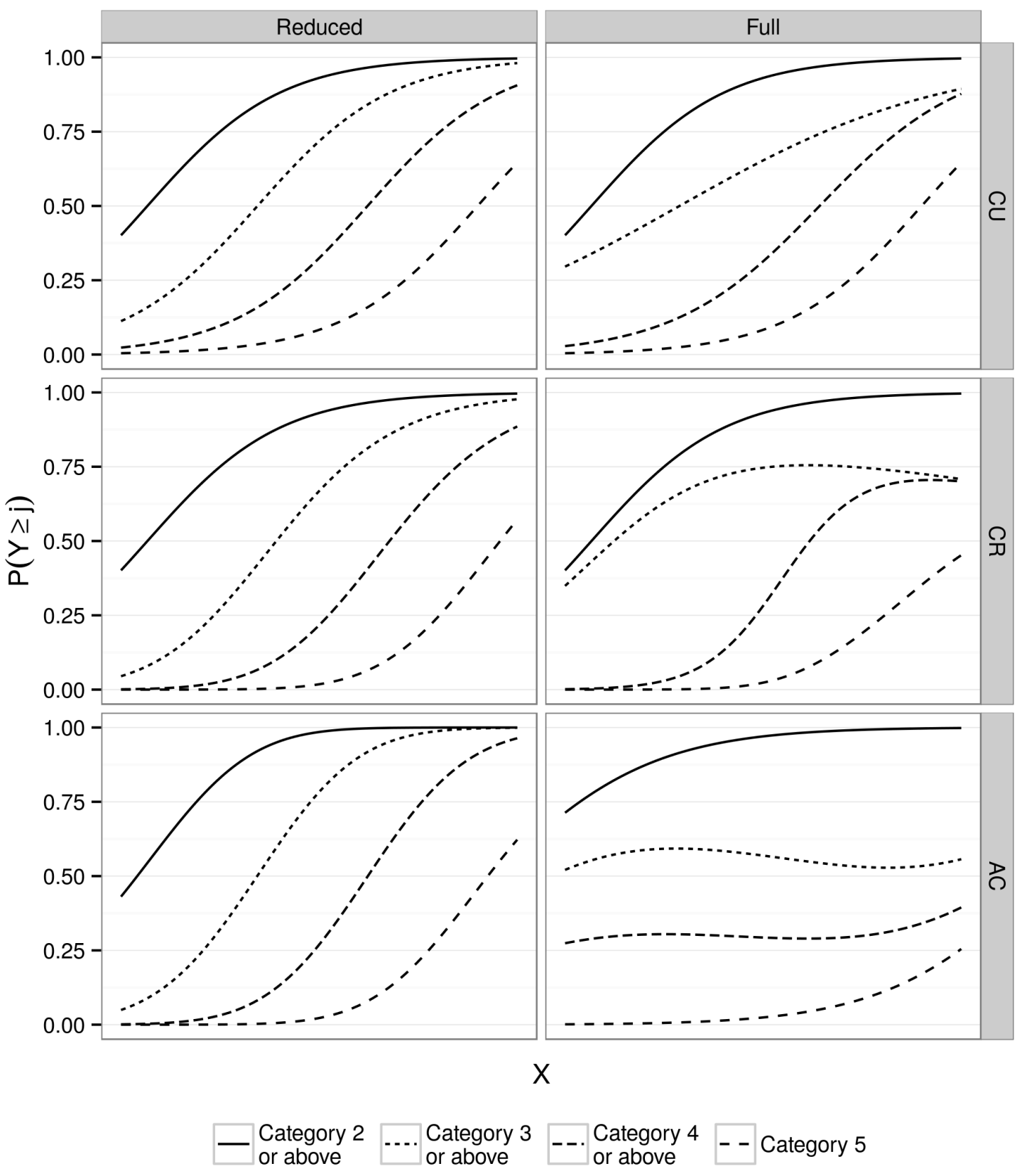

Figure 1.1: Example of probability curves for reduced and full models (in the left and right panels respectively) from each of the cumulative, continuation-ratio and adjacent categories families (in the top, middle, bottom panels respectively). 
Similar to the situation where the CUF model was reduced to CUR, we refer to the continuation-ratio link model with a shared effect as CRR.

The continuation-ratio family utilizes the link function in such a way that not all the cumulative probability curves are from the class of functions determined by the link. In fact, only one of these $C-1$ curves lie within the link's class of functions, while the other $C-2$ have a different form but still result in a similar shape curve. The middle panel of Figure 1.1 illustrates the difference between the CRF and CRR models; note that curves corresponding to individual effects $\boldsymbol{\beta}_{j}$ in the CRF are not required to be monotone. This figure also displays the differences between the CR and other families.

\subsubsection{Adjacent Categories (AC) Link Models}

The final family we consider is the adjacent categories (AC) family, which is defined

by $\gamma_{j}(\mathbf{x})=P(Y=j \mid Y=j$ or $Y=j+1, \mathbf{x})$. With this family, the models (1.2) and (1.3) have the form

$$
g[P(Y=j \mid Y=j \text { or } Y=j+1, \mathbf{x})]=\alpha_{j}+\boldsymbol{\beta}_{j}^{\top} \mathbf{x}, \quad j=1, \ldots, C-1
$$

and

$$
g[P(Y=j \mid Y=j \text { or } Y=j+1, \mathbf{x})]=\alpha_{j}+\boldsymbol{\beta}^{\top} \mathbf{x}, \quad j=1, \ldots, C-1
$$

respectively. We refer to the former expression as the full adjacent categories model $(\mathrm{ACF})$ and the latter, which has a single shared effect, as the reduced adjacent categories link model (ACR). 
Figure 1.1 may again be referred to for example curves, with the AC family appearing in the bottom panel. With this family, the differences between modeling with reduced and full models become more apparent. Each individual curve of ACF corresponds to a separate effect $\boldsymbol{\beta}_{j}$; note that each may be determined from knowledge about only two response categories and so are more flexible in the shapes they can obtain than the other models we have presented.

\subsection{Maximum Likelihood Estimation}

Suppose, for each of $i=1, \ldots, n$ individuals, that we have a $C$-category ordinal

random variable, $Y_{i}$, and a vector of covariates, $\mathbf{x}_{i}$. If $\pi_{i j}=P\left(Y_{i}=j \mid \mathbf{x}_{i}\right)$ and $\boldsymbol{\pi}_{i}=\left(\pi_{i 1}, \ldots, \pi_{i C}\right)^{\top}$ then $Y_{i}$ has a multinomial probability distribution with parameter $\boldsymbol{\pi}_{i}, Y_{i} \sim \operatorname{Multinomial}\left(1, \boldsymbol{\pi}_{i}\right)$. We can express an observation from $Y_{i}$ by a multinomial indicator vector,

$$
\mathbf{y}_{i}=\left(y_{i 1}, \ldots, y_{i C}\right)^{\top}
$$

where each component

$$
y_{i j}= \begin{cases}1, & \text { observation } i \text { is from category } j \\ 0, & \text { otherwise }\end{cases}
$$

indicates whether the $i$-th observation was from category $j$ or not. The probability mass function for $Y_{i}$ is

$$
f\left(\mathbf{y}_{i} ; \boldsymbol{\pi}_{i}\right)=\prod_{j=1}^{C} \pi_{i j}^{y_{i j}}
$$

where $\sum_{j=1}^{C} y_{i j}=1$. 
In all models presented in Section 1.2, we can use the maximum likelihood approach to obtain the maximum likelihood estimate (MLE), $\hat{\boldsymbol{\theta}}$, of the parameter vector $\boldsymbol{\theta}$. Considering $\boldsymbol{\pi}_{i}=\boldsymbol{\pi}_{i}(\boldsymbol{\theta})$ as a function of $\boldsymbol{\theta}$, we have the likelihood,

$$
l(\boldsymbol{\theta})=\prod_{i=1}^{n} f\left(\mathbf{y}_{i} ; \boldsymbol{\pi}_{i}(\boldsymbol{\theta})\right)=\prod_{i=1}^{n} \prod_{j=1}^{C} \pi_{i j}^{y_{i j}}
$$

and the log-likelihood,

$$
l(\boldsymbol{\theta})=\log l(\boldsymbol{\theta})=\sum_{i=1}^{n} \sum_{j=1}^{C} y_{i j} \log \pi_{i j}
$$

where $\boldsymbol{\theta}$ is a parameter vector of length $q$. The gradient of the log-likelihood,

$$
\frac{\partial l(\boldsymbol{\theta})}{\partial \boldsymbol{\theta}}=\sum_{i=1}^{n} \sum_{j=1}^{C} y_{i j} \frac{\partial \log \pi_{i j}}{\partial \boldsymbol{\theta}}
$$

is a row vector of length $q$ with transpose $\left[\frac{\partial}{\partial \boldsymbol{\theta}} l(\boldsymbol{\theta})\right]^{\top}=\frac{\partial}{\partial \boldsymbol{\theta}^{\top}} l(\boldsymbol{\theta})$ and the Hessian,

$$
\frac{\partial^{2} l(\boldsymbol{\theta})}{\partial \boldsymbol{\theta} \partial \boldsymbol{\theta}^{\boldsymbol{\top}}}=\sum_{i=1}^{n} \sum_{j=1}^{C} y_{i j} \frac{\partial^{2} \log \pi_{i j}}{\partial \boldsymbol{\theta} \partial \boldsymbol{\theta}^{\boldsymbol{\top}}}
$$

is a matrix of size $q \times q$.

We use both the gradient (1.12) and the Hessian (1.13) to assist in finding the maximum of the log-likelihood. The standard Newton-Raphson update for maximizing the log-likelihood is

$$
\boldsymbol{\theta}_{[t+1]}=\boldsymbol{\theta}_{[t]}-\left[\frac{\partial^{2} l\left(\boldsymbol{\theta}_{[t]}\right)}{\partial \boldsymbol{\theta} \partial \boldsymbol{\theta}^{\top}}\right]^{-1} \frac{\partial l\left(\boldsymbol{\theta}_{[t]}\right)}{\partial \boldsymbol{\theta}^{\top}}
$$

where $\boldsymbol{\theta}_{[t]}$ is the current estimate of $\boldsymbol{\theta}$, and $\boldsymbol{\theta}_{[0]}$ is an initial estimate. Note that (1.14) 
involves the observed Fisher information matrix, $\mathcal{I}\left(\boldsymbol{\theta}_{[t]}\right)=-\frac{\partial^{2} l\left(\boldsymbol{\theta}_{[t]}\right)}{\partial \boldsymbol{\theta} \partial \boldsymbol{\theta}^{\top}}$, evaluated at $\boldsymbol{\theta}_{[t]}$. Thus we can write the update as

$$
\boldsymbol{\theta}_{[t+1]}=\boldsymbol{\theta}_{[t]}-\mathcal{I}\left(\boldsymbol{\theta}_{[t]}\right)^{-1} \frac{\partial l\left(\boldsymbol{\theta}_{[t]}\right)}{\partial \boldsymbol{\theta}^{\top}}
$$

We stop the iterative procedure when the current estimate is close to the updated estimate, for example when the Euclidean norm of $\frac{\partial}{\partial \boldsymbol{\theta}^{\top}} l\left(\boldsymbol{\theta}_{[t]}\right)$ is less than some tolerance level.

An alternative to the Newton-Raphson method is the Fisher Scoring method. In (1.14) we may replace the observed information at $\boldsymbol{\theta}_{[t]}$ with the expected Fisher information at $\boldsymbol{\theta}_{[t]}$ to get the Fisher scoring update

$$
\boldsymbol{\theta}_{[t+1]}=\boldsymbol{\theta}_{[t]}+\mathcal{J}\left(\boldsymbol{\theta}_{[t]}\right)^{-1} \frac{\partial l\left(\boldsymbol{\theta}_{[t]}\right)}{\partial \boldsymbol{\theta}^{\top}}
$$

This method is asymptotically equivalent to the Newton-Raphson method.

To evaluate either the Newton-Raphson update in (1.14) or the Fisher scoring update in (1.15), it will be useful to use matrix notation for $\boldsymbol{\eta}_{i}=\left(\eta_{i 1}, \ldots, \eta_{i, C-1}\right)^{\top}$ and $\gamma_{i}=\left(\gamma_{i 1}, \ldots, \gamma_{i, C-1}\right)^{\top}$, where $\eta_{i j}=\eta_{j}\left(\mathbf{x}_{i}\right)$ and $\gamma_{i j}=\gamma_{j}\left(\mathbf{x}_{i}\right)$. For the full model (1.2) we define the $C-1 \times(C-1)(r+1)$ matrix $\mathbf{U}^{[F]}(\mathbf{x})=\left[\mathbf{I}_{C-1} \mid \mathbf{I}_{C-1} \otimes \mathbf{x}^{\top}\right]$, where $\mathbf{I}_{C-1}$ represents the identity matrix of size $C-1 \times C-1$ and the operator $\otimes$ is the Kronecker product. For the reduced model (1.3) we define $\mathbf{U}^{[R]}(\mathbf{x})=\left[\mathbf{I}_{C-1} \mid \mathbf{1}_{C-1} \otimes \mathbf{x}^{\top}\right]$, where $\mathbf{1}_{C-1}$ is a vector of ones of length $C-1$. We then have that

$$
\mathbf{U}^{[F]}(\mathbf{x}) \boldsymbol{\theta}^{[F]}=\left[\begin{array}{c}
\alpha_{1}+\boldsymbol{\beta}_{1}^{\top} \mathbf{x} \\
\vdots \\
\alpha_{C-1}+\boldsymbol{\beta}_{C-1}^{\top} \mathbf{x}
\end{array}\right]
$$


and also

$$
\mathbf{U}^{[R]}(\mathbf{x}) \boldsymbol{\theta}^{[R]}=\left[\begin{array}{c}
\alpha_{1}+\boldsymbol{\beta}^{\boldsymbol{\top}} \mathbf{x} \\
\vdots \\
\alpha_{C-1}+\boldsymbol{\beta}^{\boldsymbol{\top}} \mathbf{x}
\end{array}\right] .
$$

We let $\mathbf{U}(\mathbf{x})=\mathbf{U}^{[F]}(\mathbf{x})$ and $\boldsymbol{\theta}=\boldsymbol{\theta}^{[F]}$ for the full model and $\mathbf{U}(\mathbf{x})=\mathbf{U}^{[R]}(\mathbf{x})$ and $\boldsymbol{\theta}=\boldsymbol{\theta}^{[R]}$ for the reduced model. We then define the matrices $\mathbf{U}_{i}=\mathbf{U}\left(\mathbf{x}_{i}\right)$ and also represent the vectors corresponding to the $j$-th column of $\mathbf{U}_{i}^{\top}$ by $\mathbf{u}_{i j}, i=1, \ldots, n$. Thus we have $\boldsymbol{\eta}_{i}=\mathbf{U}_{i} \boldsymbol{\theta}$ and $\frac{\partial}{\partial \boldsymbol{\theta}} \boldsymbol{\eta}_{i}=\mathbf{U}_{i}$

Note that regardless of which family is used, both the full model (1.2) and the reduced model (1.3) relate $\boldsymbol{\gamma}_{i}$ to $\boldsymbol{\eta}_{i}$ through the link $g$. We denote the inverse link function by $g^{-1}$ and its first and second derivatives by $\left[g^{-1}\right]^{\prime}$ and $\left[g^{-1}\right]^{\prime \prime}$ respectively. Rearranging (1.1) gives $\gamma_{i j}=g\left(\eta_{i j}\right)^{-1}$ and we have that

$$
\mathbf{E}_{i}=\frac{\partial \boldsymbol{\gamma}_{i}}{\partial \boldsymbol{\eta}_{i}}=\operatorname{diag}\left\{\left[g\left(\eta_{i 1}\right)^{-1}\right]^{\prime}, \ldots,\left[g\left(\eta_{i, C-1}\right)^{-1}\right]^{\prime}\right\}
$$

is a $C-1 \times C-1$ diagonal matrix. Finally, we have

$$
\frac{\partial \log \pi_{i j}}{\partial \boldsymbol{\theta}}=\frac{\partial \log \pi_{i j}}{\partial \boldsymbol{\gamma}_{i}}\left(\frac{\partial \boldsymbol{\gamma}_{i}}{\partial \boldsymbol{\eta}_{i}}\right)\left(\frac{\partial \boldsymbol{\eta}_{i}}{\partial \boldsymbol{\theta}}\right)=\frac{\partial \log \pi_{i j}}{\partial \boldsymbol{\gamma}_{i}} \mathbf{E}_{i} \mathbf{U}_{i}
$$


and

$$
\begin{aligned}
\frac{\partial^{2} \log \pi_{i j}}{\partial \boldsymbol{\theta} \partial \boldsymbol{\theta}^{\top}} & =\frac{\partial \boldsymbol{\gamma}_{i}}{\partial \boldsymbol{\eta}_{i}^{\top}}\left(\frac{\partial \boldsymbol{\eta}_{i}}{\partial \boldsymbol{\theta}^{\top}}\right)\left(\frac{\partial^{2} \log \pi_{i j}}{\partial \boldsymbol{\gamma} \partial \boldsymbol{\gamma}^{\top}}\right)\left(\frac{\partial \boldsymbol{\gamma}_{i}}{\partial \boldsymbol{\eta}_{i}}\right) \frac{\partial \boldsymbol{\eta}_{i}}{\partial \boldsymbol{\theta}}+\sum_{k=1}^{C-1}\left(\frac{\partial \log \pi_{i j}}{\partial \gamma_{i k}}\right) \frac{\partial^{2} \gamma_{i k}}{\partial \boldsymbol{\theta} \partial \boldsymbol{\theta}^{\top}} \\
& =\mathbf{U}_{i}^{\boldsymbol{\top}} \mathbf{E}_{i}\left(\frac{\partial^{2} \log \pi_{i j}}{\partial \boldsymbol{\gamma} \partial \boldsymbol{\gamma}^{\top}}\right) \mathbf{E}_{i} \mathbf{U}_{i}+\sum_{k=1}^{C-1}\left(\frac{\partial \log \pi_{i j}}{\partial \gamma_{i k}}\right)\left[g\left(\eta_{i k}\right)^{-1}\right]^{\prime \prime} \mathbf{u}_{i k} \mathbf{u}_{i k}^{\top} .
\end{aligned}
$$

We use (1.19) and (1.20) in the optimization procedure, however, to fully evaluate Equations (1.11), (1.12) and (1.13), we still require expressions for $\log \pi_{i j}$ as well

as its first and second derivatives with respect to $\gamma_{i}, \frac{\partial}{\partial \gamma_{i}} \log \pi_{i j}$ and $\frac{\partial^{2}}{\partial \gamma_{i} \partial \gamma_{i}^{\top}} \log \pi_{i j}$, respectively. Since these final expressions are family-specific, we give details for the $\mathrm{CU}, \mathrm{CR}$ and $\mathrm{AC}$ families in the remainder of this section.

\subsubsection{Cumulative (CU) Link Models}

For the CU family, the transformation from the cumulative probabilities $\gamma_{i}$ to the category probabilities $\boldsymbol{\pi}_{i}$ is linear. If we let $\mathbf{M}$ be a lower triangular matrix of size $C-1 \times C-1$ with ones on and below the diagonal and zeros elsewhere, then we can construct a matrix, $\mathbf{T}=[\mathbf{M} \mid \mathbf{0}]$, by augmenting $\mathbf{M}$ by a column of zeros. This matrix $\mathbf{T}$ defines the linear transformation for the $\mathrm{CU}$ family, $\boldsymbol{\gamma}_{i}=\mathbf{T} \boldsymbol{\pi}_{i}$. The inverse transformation is given by $\boldsymbol{\pi}_{i}=\mathbf{s}+\mathbf{T}^{-} \boldsymbol{\gamma}_{i}$ where

$$
\mathbf{s}=\left[\begin{array}{c}
0 \\
0 \\
\vdots \\
0 \\
\overline{1}
\end{array}\right] \quad \text { and } \quad \mathbf{T}^{-}=\left[\begin{array}{rrrr}
1 & & & 0 \\
-1 & 1 & & \\
\ddots & \ddots & \\
0 & -1 & 1 \\
\hline 0 & \cdots & 0 & -1
\end{array}\right]
$$


are of length $C$ and size $C \times C-1$ respectively. Note that $\mathbf{T} \mathbf{T}^{-}=\mathbf{I}_{C-1}$ and so $\mathbf{T}$ is a generalized inverse of $\mathbf{T}^{-}$. We now have

$$
\frac{\partial \log \boldsymbol{\pi}_{i}}{\partial \boldsymbol{\gamma}_{i}}=\frac{\partial \log \boldsymbol{\pi}_{i}}{\partial \boldsymbol{\pi}_{i}}\left(\frac{\partial \boldsymbol{\pi}_{i}}{\partial \boldsymbol{\gamma}_{i}}\right)=\operatorname{diag}\left\{\frac{1}{\pi_{i 1}}, \ldots, \frac{1}{\pi_{i C}}\right\} \mathbf{T}^{-}
$$

and

$$
\frac{\partial^{2} \log \pi_{i j}}{\partial \boldsymbol{\gamma}_{i} \partial \boldsymbol{\gamma}_{i}^{\top}}=\left[\mathbf{T}^{-}\right]^{\top} \operatorname{diag}\left\{-\left(\frac{1}{\pi_{i 1}}\right)^{2}, \ldots,-\left(\frac{1}{\pi_{i C}}\right)^{2}\right\} \mathbf{T}^{-}
$$

which can be used in (1.19) and (1.20).

\subsubsection{Continuation-Ratio (CR) Link Models}

In Section 1.2.2 we defined the CR family in terms of the conditional response prob-

abilities $\gamma_{j}(\mathbf{x})=P(Y=j \mid Y \geq j, \mathbf{x})$. However, to evaluate the likelihood we need the unconditional response probabilities, which we give below.

$$
\begin{aligned}
\pi_{i j}=\left\{\begin{array}{cl}
P\left(Y_{i}=1 \mid Y_{i} \geq 1, \mathbf{x}\right) & , j=1 \\
P\left(Y_{i}=j \mid Y_{i} \geq j, \mathbf{x}\right) \prod_{k=1}^{j-1}\left[1-P\left(Y_{i}=k \mid Y_{i} \geq k, \mathbf{x}\right)\right] & , j=2, \ldots, C
\end{array}\right. \\
=\left\{\begin{array}{cl}
\gamma_{i 1} & , j=1 \\
\gamma_{i j} \prod_{k=1}^{j-1}\left(1-\gamma_{i k}\right) & , j=2, \ldots, C-1 \\
\prod_{k=1}^{C-1}\left(1-\gamma_{i k}\right) & , j=C
\end{array}\right.
\end{aligned}
$$


We let $\mathbf{A}=\left[\frac{\mathbf{I}_{C-1}}{\mathbf{0}_{C-1}^{\top}}\right]$ and $\mathbf{B}=\left[\frac{\mathbf{0}_{C-1}^{\top}}{\mathbf{M}}\right]$, and $\mathbf{a}_{j}$ and $\mathbf{b}_{j}$ denote the vectors corresponding to the $j$-th columns of $\mathbf{A}^{\top}$ and $\mathbf{B}^{\top}$ respectively. Thus, for CR models, we have the log-response probabilities

$$
\log \pi_{i j}=\mathbf{a}_{j}^{\top} \log \gamma_{i}-\mathbf{b}_{j}^{\top} \log \left(1-\gamma_{i}\right),
$$

along with their gradient

$$
\frac{\partial \log \pi_{i j}}{\partial \boldsymbol{\gamma}_{i}}=\mathbf{a}_{j}^{\top} \operatorname{diag}\left\{\frac{1}{\gamma_{i 1}}, \ldots, \frac{1}{\gamma_{i, C-1}}\right\}-\mathbf{b}_{j}^{\top} \operatorname{diag}\left\{\frac{1}{1-\gamma_{i 1}}, \ldots, \frac{1}{1-\gamma_{i, C-1}}\right\}
$$

and Hessian

$$
\begin{aligned}
\frac{\partial^{2} \log \pi_{i j}}{\partial \boldsymbol{\gamma}_{i} \partial \boldsymbol{\gamma}_{i}^{\top}}=-\operatorname{diag}\left\{\mathbf{a}_{j}^{\top}\right\} & \operatorname{diag}\left\{\left(\frac{1}{\gamma_{i 1}}\right)^{2}, \ldots,\left(\frac{1}{\gamma_{i, C-1}}\right)^{2}\right\} \\
& -\operatorname{diag}\left\{\mathbf{b}_{j}^{\top}\right\} \operatorname{diag}\left\{\left(\frac{1}{1-\gamma_{i 1}}\right)^{2}, \ldots,\left(\frac{1}{1-\gamma_{i, C-1}}\right)^{2}\right\} .
\end{aligned}
$$

These latter two equations can be used in (1.19) and (1.20) respectively.

\subsubsection{Adjacent Categories (AC) Link Models}

In Section 1.2.3 we defined the AC family in terms of the conditional response probabilities $\gamma_{j}(\mathbf{x})=P\left(Y_{i}=j \mid Y_{i}=j\right.$ or $\left.Y_{i}=j+1, \mathbf{x}\right)$. In this section we find the unconditional response probabilities and their first and second derivatives with respect 
to $\gamma_{i}$. First, if we let $h_{i j}=\sum_{k=j}^{C-1} \operatorname{logit}\left(\gamma_{i k}\right)$ then we have

$$
\frac{\partial h_{i j}}{\partial \gamma_{i s}}=\left\{\begin{array}{cl}
\operatorname{logit}\left(\gamma_{i j}\right)\left[1-\operatorname{logit}\left(\gamma_{i j}\right)\right] & , j=s \\
0 & , \text { otherwise }
\end{array}\right.
$$

and

$$
\frac{\partial^{2} h_{i j}}{\partial \gamma_{i s} \partial \gamma_{i t}}=\left\{\begin{array}{cc}
\operatorname{logit}\left(\gamma_{i j}\right)\left(1-2 \operatorname{logit}\left(\gamma_{i j}\right)\left[1-2 \operatorname{logit}\left(\gamma_{i j}\right)\right]\right) & , j=s, j=t \\
0 & , \text { otherwise }
\end{array}\right.
$$

We now note that

$$
\log \left(\frac{\pi_{i j}}{\pi_{i C}}\right)=\log \left(\prod_{k=j}^{C-1} \frac{\pi_{i k}}{\pi_{i, k+1}}\right)=\sum_{k=j}^{C-1} \log \left(\frac{\pi_{i k}}{\pi_{i, k+1}}\right)=\sum_{k=j}^{C-1} \operatorname{logit}\left(\gamma_{i k}\right)=h_{i j}
$$

and so

$$
\log \pi_{i j}=\log \pi_{i C}+h_{i j}
$$

for all $j=1, \ldots, C-1$. Hence

$$
\pi_{i j}=\exp \left(\log \pi_{i C}+h_{i j}\right)=\pi_{i C} \exp \left(h_{i j}\right), j=1, \ldots, C-1
$$

and since

$$
1=\sum_{j=1}^{C} \pi_{i j}=\pi_{i C}\left\{1+\sum_{j=1}^{C-1} \exp \left(h_{i j}\right)\right\}
$$


we have

$$
\log \pi_{i C}=-\log \left[1+\sum_{j=1}^{C-1} \exp \left(h_{i j}\right)\right] .
$$

Finally, we have

$$
\frac{\partial \log \pi_{i C}}{\partial \gamma_{i s}}=-\frac{1}{1+\sum_{j=1}^{C-1} \exp \left(h_{i j}\right)} \sum_{j=1}^{C-1} \exp \left(h_{i j}\right) \frac{\partial h_{i j}}{\partial \gamma_{i s}}
$$

and

$$
\begin{aligned}
\frac{\partial^{2} \log \pi_{i C}}{\partial \gamma_{i s} \partial \gamma_{i t}}=-\frac{1}{1+\sum_{j=1}^{C-1} \exp \left(h_{i j}\right)} \sum_{j=1}^{C-1}\left\{\exp \left(h_{i j}\right) \frac{\partial^{2} h_{i j}\left(\gamma_{i}\right)}{\partial \gamma_{i s} \partial \gamma_{i t}}+\frac{\partial h_{i j}}{\partial \gamma_{i s}} \exp \left(h_{i j}\right) \frac{\partial h_{i j}}{\partial \gamma_{i t}}\right\} \\
+\left(\frac{1}{1+\sum_{j=1}^{C-1} \exp \left(h_{i j}\right)}\right)^{2} \sum_{j=1}^{C-1} \exp \left(h_{i j}\right) \frac{\partial h_{i j}}{\partial \gamma_{i t}}
\end{aligned}
$$

which are the gradient and Hessian of (1.22) respectively, and similarly the gradient

$$
\frac{\partial \log \pi_{i j}}{\partial \gamma_{i s}}=\frac{\partial \log \pi_{i C}}{\partial \gamma_{i s}}+\frac{\partial h_{i j}}{\partial \gamma_{i s}}
$$

and Hessian

$$
\frac{\partial^{2} \log \pi_{i j}}{\partial \gamma_{i s} \partial \gamma_{i t}}=\frac{\partial^{2} \log \pi_{i C}}{\partial \gamma_{i s} \partial \gamma_{i t}}+\frac{\partial^{2} h_{i j}}{\partial \gamma_{i s} \partial \gamma_{i t}}
$$

of (1.21). We use these expressions to evaluate (1.19) and (1.20) for AC models. 


\subsection{Background Response}

A common model for quantal dose-response is one that utilizes the Hill equation introduced by Hill (1910). We write the model as

$$
P_{\mathrm{HILL}}(\text { response } \mid d)=\zeta+(1-\zeta)\left(\frac{d^{\delta}}{\kappa^{\delta}+d^{\delta}}\right),
$$

where $d$ is the dose, $\zeta$ is a parameter describing the background response, $\kappa$ and $\delta$ are the location and shape parameters respectively for the model. By examining the model it is apparent that the fitted probabilities will be at least $\zeta$; thus $\zeta$ imposes a lower bound on the response (specifically when $d=0$ ). Unlike the models in the previous section, this lower bound can be non-zero. We can consider this as a background level of response. This provides useful flexibility to the model, since a member of the control (placebo) group can be observed to respond with non-zero probability. Keeping this general notion of added model flexibility in mind, in this section we present a general extension of ordinal models to incorporate a background response.

\subsubsection{Incorporating a Background Response With a Latent Process}

Following the approach in Xie and Simpson (1999), we describe a latent process to incorporate a background or spontaneous response in ordinal models. We suppose that a response in any category can either occur spontaneously, or due to exposure to some explanatory variables and refer to these as spontaneous and exposure responses, respectively. We let

$$
W \sim \text { Multinomial }(1, \boldsymbol{\tau})
$$


be a random variable corresponding to spontaneous response where the vector $\boldsymbol{\tau}=$ $\left(\tau_{1}, \ldots, \tau_{C}\right)^{\top}$ contains the probabilities of the spontaneous response in each category. In addition, we assume that $\boldsymbol{\tau}=\boldsymbol{\tau}(\boldsymbol{\zeta})$ is a vector-valued function of some parameter vector $\boldsymbol{\zeta}$ (of length up to $C-1$ ), so that $\tau_{j}=\tau_{j}(\boldsymbol{\zeta})=P(W=j$ ). Note that for the probabilities to be valid we must have $\sum_{j=1}^{C} \tau_{j}(\boldsymbol{\zeta})=1$.

We also let

$$
\tilde{Y} \mid \mathbf{x} \sim \operatorname{Multinomial}(1, \tilde{\boldsymbol{\pi}}(\mathbf{x}))
$$

where $\tilde{\boldsymbol{\pi}}(\mathbf{x})=\left(\tilde{\pi}_{1}(\mathbf{x}), \ldots, \tilde{\pi}_{C}(\mathbf{x})\right)^{\top}$ and $\tilde{\pi}_{j}(\mathbf{x})=P(\tilde{Y}=j \mid \mathbf{x})$ be a random variable corresponding to exposure response. Here $\tilde{\boldsymbol{\pi}}(\mathbf{x})$ is a vector of category probabilities for the exposure response at predictor $\mathbf{x}$ given by some ordinal model parameterized by $\tilde{\boldsymbol{\theta}}$. This exposure model includes those models presented in Section 1.2, but we are not limited to only those models here.

We will assume that these two random variables, $W$ and $\tilde{Y} \mid \mathbf{x}$, corresponding to the spontaneous and exposure responses respectively, are independent. We let the observed response be the maximum of the spontaneous and exposure responses, $Y=\max \{W, \tilde{Y}\}$. We then have the following model for the response variable $Y$,

$$
\begin{aligned}
P(Y \leq j \mid \mathbf{x}) & =P(\max \{W, \tilde{Y}\} \leq j \mid \mathbf{x}) \\
& =P(W \leq j, \tilde{Y} \leq j \mid \mathbf{x}) \\
& =P(W \leq j \mid \mathbf{x}) P(\tilde{Y} \leq j \mid \mathbf{x}) \\
& =\left(\sum_{k=1}^{j} \tau_{k}\right)\left(\sum_{k=1}^{j} \tilde{\pi}_{k}(\mathbf{x})\right), j=1, \ldots, C-1 .
\end{aligned}
$$

Since $\boldsymbol{\tau}$ is a function of $\boldsymbol{\zeta}$ and $\tilde{\boldsymbol{\pi}}$ a function of $\tilde{\boldsymbol{\theta}}$, the full parameter vector for the background model is $\boldsymbol{\theta}=\left(\boldsymbol{\zeta}^{\top}, \tilde{\boldsymbol{\theta}}^{\top}\right)^{\top}$. 
When the length of $\boldsymbol{\zeta}$ is $C-1$, this model allows the spontaneous response rate for all categories to be fully specified. However, we can make the model more parsimonious by employing a simplifying assumption. For example, if we assume that the first category is associated with the lowest severity level, then $\boldsymbol{\tau}(\zeta)=\left(1-\zeta, \frac{\zeta}{C-1}, \ldots, \frac{\zeta}{C-1}\right)^{\top}$ is one reasonable simplifying assumption. This function implies that when a spontaneous response occurs, it will occur in the first category with probability $1-\zeta$, and in any of the other $C-1$ response categories with equal probability. Under this assumption, our model becomes

$$
P(Y \leq j \mid \mathbf{x})=\left(1-\zeta \frac{C-j}{C-1}\right) \sum_{k=1}^{j} \tilde{\pi}_{k}(\mathbf{x}), \quad j=1, \ldots, C-1
$$

When using CUR, CRR and ACR as models for the exposure response probabilities $\tilde{\boldsymbol{\pi}}(\mathbf{x})$ in the latent process, we refer to these as CURB, CRRB and ACRB respectively; we add a "B" to the acronym to indicate that a background parameter vector is included. It is also possible to use the models with separate effects CUF, $\mathrm{CRF}$ and $\mathrm{ACF}$, which we refer to as $\mathrm{CUFB}, \mathrm{CRFB}$ and $\mathrm{ACFB}$ respectively. However, using models with separate effects in combination with a background parameter often results in a model with too much flexibility.

The plots in Figure 1.2 illustrate some examples of the models with background response for the $\mathrm{CU}, \mathrm{CR}$ and $\mathrm{AC}$ families. The effect of the additional parameter in the background models is that the cumulative probabilities must approach either zero or one as the covariates approach either positive or negative infinity, but not both. In practical terms this allows for a more flexible fit than the models in Section 1.2.

Note that for quantal responses, the CURB model of the log dose with a logit link simply reduces to the Hill model given in (1.23). Specifically, if we let $x=\log d$, 


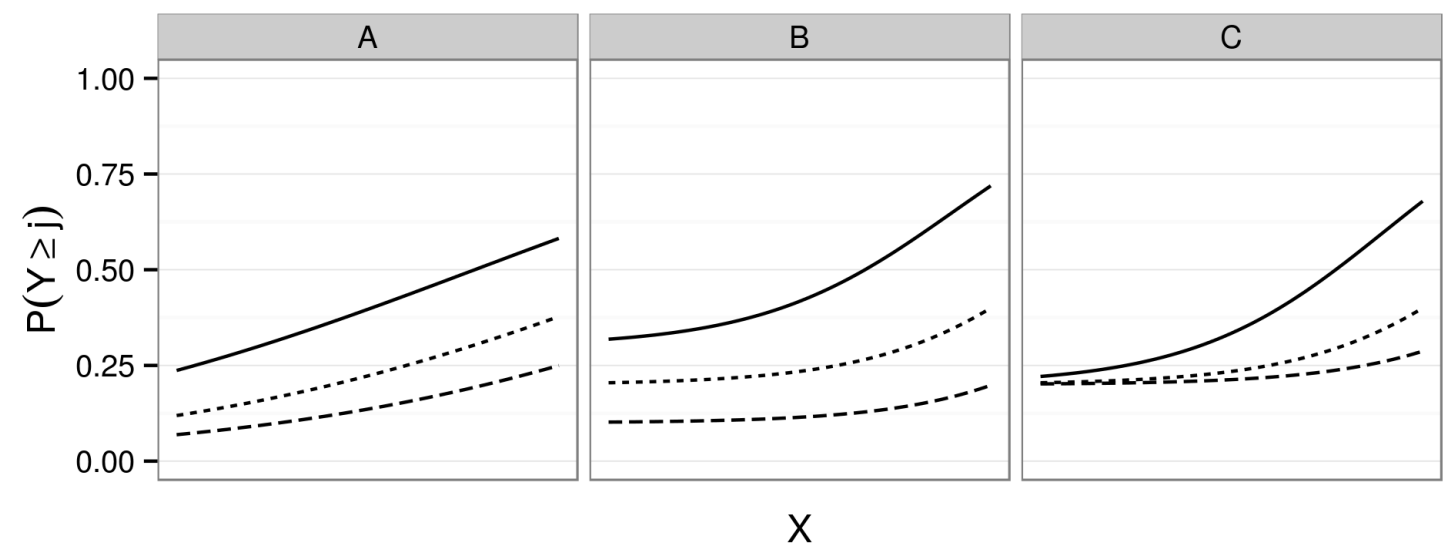

— $\begin{array}{lll}\text { Category } 2 & \ldots & \text { Category } 3 \\ \text { or above } & \ldots-. & \text { Category } 4 \\ \text { or above }\end{array}$

Figure 1.2: Example of probability curves for CURB where: $\boldsymbol{\tau}=(1,0,0,0)^{\top}$ in panel A (which is equivalent to CUR); $\boldsymbol{\tau}=(0.7,0.1,0.1,0.1)^{\top}$ in panel B; and $\boldsymbol{\tau}=(0.8,0,0,0.2)^{\mathrm{\top}}$ in panel C.

$\beta=-\delta$ and $\alpha=\delta \log \kappa$ then for CUR in (1.2.1) we have that

$$
\begin{aligned}
P_{\mathrm{CUR}}(Y=2 \mid x) & =1-\operatorname{logit}^{-1}\left(\alpha_{1}+\beta x\right) \\
& =\operatorname{logit}^{-1}\left[-\left(\alpha_{1}+\beta x\right)\right] \\
& =\operatorname{logit}{ }^{-1}[-(\delta \log \kappa-\delta \log d)] \\
& =\frac{\kappa^{-\delta} d^{\delta}}{1+\kappa^{-\delta} d^{\delta}} \\
& =\frac{d^{\delta}}{\kappa^{\delta}+d^{\delta}},
\end{aligned}
$$


and additionally, if $\tau(\zeta)=\zeta$ is the identity function, then

$$
\begin{aligned}
P_{\mathrm{CURB}}(\text { response } \mid x) & =\zeta+(1-\zeta) P_{\mathrm{CUR}}(\text { response } \mid x) \\
& =\zeta+(1-\zeta)\left(\frac{d^{\delta}}{\kappa^{\delta}+d^{\delta}}\right) \\
& =P_{\mathrm{HILL}}(\text { response } \mid d) .
\end{aligned}
$$

\subsubsection{Estimation of Background Model Parameters}

Suppose that for each individual $i=1, \ldots, n$, in addition to $Y_{i}$, there exists a pair of latent variables $\left\{W_{i}, \tilde{Y}_{i}\right\}$, which correspond to the spontaneous response and exposure responses respectively for that individual. We assume that $W_{i}$ and $\tilde{Y}_{i}$ are independent and $W_{i} \sim \operatorname{Multinomial}(1, \boldsymbol{\tau})$ and $\tilde{Y}_{i} \mid \mathbf{x}_{i} \sim \operatorname{Multinomial}\left(1, \tilde{\boldsymbol{\pi}}\left(\mathbf{x}_{i}\right)\right)$.

The exposure response probabilities for the $i$-th individual and $j$-th category is $\tilde{\pi}_{i j}=P(\tilde{Y}=j \mid \mathbf{x})$ and $\tilde{\pi}_{i}=\left(\tilde{\pi}_{i 1}, \ldots, \tilde{\pi}_{i C}\right)^{\top}$ is the vector of exposure response probabilities for that individual. Using these probabilities, along with the spontaneous response probabilities, $\boldsymbol{\tau}$, we can calculate the response probabilities, $\boldsymbol{\pi}_{i}=\boldsymbol{\pi}\left(\mathbf{x}_{i}\right)$, for the $i$-th individual given by the background model (1.24). Specifically, we have that

$$
\begin{aligned}
\pi_{i j} & =P\left(Y_{i} \leq j \mid \mathbf{x}_{i}\right)-P\left(Y_{i} \leq j-1 \mid \mathbf{x}_{i}\right) \\
& =\left(\sum_{k=1}^{j} \tau_{k}\right)\left(\sum_{k=1}^{j} \tilde{\pi}_{i k}\right)-\left(\sum_{k=1}^{j-1} \tau_{k}\right)\left(\sum_{k=1}^{j-1} \tilde{\pi}_{i k}\right)
\end{aligned}
$$

for $j=2, \ldots, C$ and also that $\pi_{i 1}=P\left(Y_{i} \leq 1 \mid \mathbf{x}_{i}\right)=\tau_{1} \tilde{\pi}_{i 1}$. Thus

$$
\pi_{i j}=\left(\sum_{k=1}^{j} \tau_{k}\right) \tilde{\pi}_{i j}+\tau_{j}\left(\sum_{k=1}^{j} \tilde{\pi}_{i k}\right)-\tau_{j} \tilde{\pi}_{i j}
$$


for $j=1, \ldots, C$ and $i=1, \ldots, n$. We also have the gradient

$$
\frac{\partial \pi_{i j}}{\partial \boldsymbol{\theta}}=\sum_{k=1}^{j} \frac{\partial}{\partial \boldsymbol{\theta}}\left(\tau_{k} \tilde{\pi}_{i j}\right)+\sum_{k=1}^{j} \frac{\partial}{\partial \boldsymbol{\theta}}\left(\tau_{j} \tilde{\pi}_{i k}\right)-\frac{\partial}{\partial \boldsymbol{\theta}}\left(\tau_{j} \tilde{\pi}_{i j}\right),
$$

where the individual components,

$$
\frac{\partial}{\partial \boldsymbol{\theta}}\left(\tau_{k} \tilde{\pi}_{i l}\right)=\left[\frac{\partial \tau_{k}}{\partial \boldsymbol{\zeta}} \tilde{\pi}_{i l} \mid \tau_{k} \frac{\partial \tilde{\pi}_{i l}}{\partial \tilde{\boldsymbol{\theta}}}\right], k, l=1, \ldots, C
$$

are functions of the exposure model, along with the background response and their derivatives. Similarly, we can write the Hessian as

$$
\frac{\partial^{2} \pi_{i j}}{\partial \boldsymbol{\theta} \partial \boldsymbol{\theta}^{\boldsymbol{\top}}}=\left[\sum_{k=1}^{j} \frac{\partial^{2}}{\partial \boldsymbol{\theta} \partial \boldsymbol{\theta}^{\boldsymbol{\top}}}\left(\tau_{k} \tilde{\pi}_{i j}\right)\right]+\left[\sum_{k=1}^{j} \frac{\partial^{2}}{\partial \boldsymbol{\theta} \partial \boldsymbol{\theta}^{\boldsymbol{\top}}}\left(\tau_{j} \tilde{\pi}_{i k}\right)\right]-\frac{\partial^{2}}{\partial \boldsymbol{\theta} \partial \boldsymbol{\theta}^{\boldsymbol{\top}}}\left(\tau_{j} \tilde{\pi}_{i j}\right)
$$

where

$$
\frac{\partial^{2}}{\partial \boldsymbol{\theta} \partial \boldsymbol{\theta}^{\boldsymbol{\top}}}\left(\tau_{k} \tilde{\pi}_{i l}\right)=\left[\begin{array}{c|c}
\frac{\partial^{2} \tau_{k}}{\partial \boldsymbol{\zeta} \partial \boldsymbol{\zeta}^{\boldsymbol{T}}} \tilde{\pi}_{i l} & \frac{\partial \tau_{k}}{\partial \boldsymbol{\zeta}^{\boldsymbol{T}}} \frac{\partial \tilde{\pi}_{i l}}{\partial \tilde{\boldsymbol{\theta}}} \\
\hline \frac{\partial \tilde{\pi}_{i l}}{\partial \tilde{\boldsymbol{\theta}}^{\boldsymbol{T}}} \frac{\partial \tau_{k}}{\partial \boldsymbol{\zeta}} & \tau_{k} \frac{\partial^{2} \tilde{\pi}_{i l}}{\partial \tilde{\boldsymbol{\theta}} \partial \tilde{\boldsymbol{\theta}}^{\boldsymbol{T}}}
\end{array}\right], k, l=1, \ldots, C .
$$

Note that for the exposure model, we require $\tilde{\pi}_{i j}$ and its derivatives; however, for some models it may be more convenient and natural to evaluate $\log \tilde{\pi}_{i j}$ and its derivatives (such as was the case in Section 1.3). In these instances we can use the following relations

$$
\frac{\partial \tilde{\pi}_{i j}}{\partial \boldsymbol{\theta}}=\tilde{\pi}_{i j} \frac{\partial \log \tilde{\pi}_{i j}}{\partial \boldsymbol{\theta}}
$$


and

$$
\frac{\partial^{2} \tilde{\pi}_{i j}}{\partial \boldsymbol{\theta} \partial \boldsymbol{\theta}^{\top}}=\tilde{\pi}_{i j}\left[\frac{\partial^{2} \log \tilde{\pi}_{i j}}{\partial \boldsymbol{\theta} \partial \boldsymbol{\theta}^{\top}}+\frac{\partial \log \tilde{\pi}_{i j}}{\partial \boldsymbol{\theta}^{\top}} \frac{\partial \log \tilde{\pi}_{i j}}{\partial \boldsymbol{\theta}}\right]
$$

to obtain the derivatives of $\tilde{\pi}_{i j}$.

\subsection{Simulation Study}

In this section, we investigate the properties of the estimators of the parameters of the models presented in Sections 1.2 and 1.4. We do so via a simulation study that is based on the following data examples.

\begin{tabular}{lcccccc}
\hline & \multicolumn{5}{c}{ Category } \\
\cline { 2 - 5 } Dose Type & Death & $\begin{array}{c}\text { Vegetative } \\
\text { State }\end{array}$ & $\begin{array}{c}\text { Major } \\
\text { Disability }\end{array}$ & $\begin{array}{c}\text { Minor } \\
\text { Disability }\end{array}$ & $\begin{array}{c}\text { Good } \\
\text { Recovery }\end{array}$ & \\
\hline Placebo & 59 & 25 & 46 & 48 & 32 & 210 \\
Low Dose & 48 & 21 & 44 & 47 & 30 & 190 \\
Medium Dose & 44 & 14 & 54 & 64 & 31 & 207 \\
High Dose & 43 & 4 & 49 & 58 & 41 & 195 \\
\hline
\end{tabular}

Table 1.1: Responses of trauma patients on the Glasgow Outcome Scale for four dose levels (Chuang-Stein and Agresti, 1997).

In Table 1.1 we reproduce a data example that originally appeared in ChuangStein and Agresti (1997). These data originate from a clinical trial where patients who experienced trauma due to subarachnoid hemorrhage were administered one of four levels of a drug (placebo, low dose, medium dose, high dose), and their outcome (death, vegetative state, major disability, minor disability and good recovery) on the 
Glasgow Outcome Scale (GOS) was recorded. One of the objectives of this trial was to investigate if more positive GOS outcomes were associated with a higher dose of the drug.

We consider as a second example the results of a study where chicken embryos were exposed to the Tinaroo virus. These data were originally reported by Jarrett et al. (1981). A subset that were republished in Morgan (1992) and Xie and Simpson (1999) are given in Table 1.2. Specifically at each of four inoculum titre levels $(3,20,2400$, 88000) of plaque-forming units/egg (PFU/egg) along with a control group, responses of counts are presented for three ordinal outcomes of "not deformed", "deformed", and "death".

\begin{tabular}{lcccc}
\hline \multirow{2}{*}{$\begin{array}{c}\text { Inoculum titre } \\
\text { (PFU/egg) }\end{array}$} & $\begin{array}{c}\text { Not } \\
\text { deformed }\end{array}$ & Deformed & Death & \\
\cline { 2 - 4 } & 17 & 0 & 1 & 18 \\
3 & 18 & 0 & 1 & 19 \\
20 & 17 & 0 & 2 & 19 \\
2400 & 2 & 9 & 4 & 15 \\
80000 & 0 & 10 & 9 & 19 \\
\hline
\end{tabular}

Table 1.2: Responses of chicken embryos exposed to the Tinaroo virus (Jarrett et al., 1981). 


\subsubsection{Glasgow Outcome Scale Data}

For each of the cumulative link, continuation-ratio, and adjacent categories families, four models were fit to the data provided in Table 1.1. These were distinguished by whether or not the effects were shared or separate, and the presence or absence of a background parameter vector. These twelve resulting fits are displayed in Figure 1.3 and the associated AICs are shown in Table 1.3.

\begin{tabular}{|c|c|c|c|c|c|c|}
\hline \multirow{2}{*}{ Model } & \multicolumn{2}{|c|}{$\mathrm{CU}$} & \multicolumn{2}{|c|}{$\mathrm{CR}$} & \multicolumn{2}{|c|}{$\mathrm{AC}$} \\
\hline & Reduced & Full & Reduced & Full & Reduced & Full \\
\hline Exposure & 2471.3 & 2463.0 & 2472.6 & 2465.0 & 2471.3 & 2465.1 \\
\hline Background & 2473.7 & 2468.1 & 2473.3 & 2468.0 & 2473.8 & 2471.5 \\
\hline
\end{tabular}

Table 1.3: AIC of the model fits to the Glasgow Outcome Scale data. Results for each of the six models in Section 1.2 are shown in the top row; the results for the corresponding background models are given in the bottom row.

Figure 1.3 shows that all these models result in comparable fits. From the AIC values in Table 1.3, we can see that the model with separate effects and no background parameter is the best in each family of models. Also, the best model overall for this data according to the AIC is CUF, however CRF and ACF provide similar fits.

To investigate the performance of these models over replications of the study design, we now consider a simulation motivated by this data example. Specifically, we generate 1,000 data sets from the data given in Table 1.1. Each of these data sets mirrored the original data setup; they consisted of the same total number of observations as in the original data set broken down by dose type. Specifically, 210 placebo observations were simulated from the multinomial probability vector 


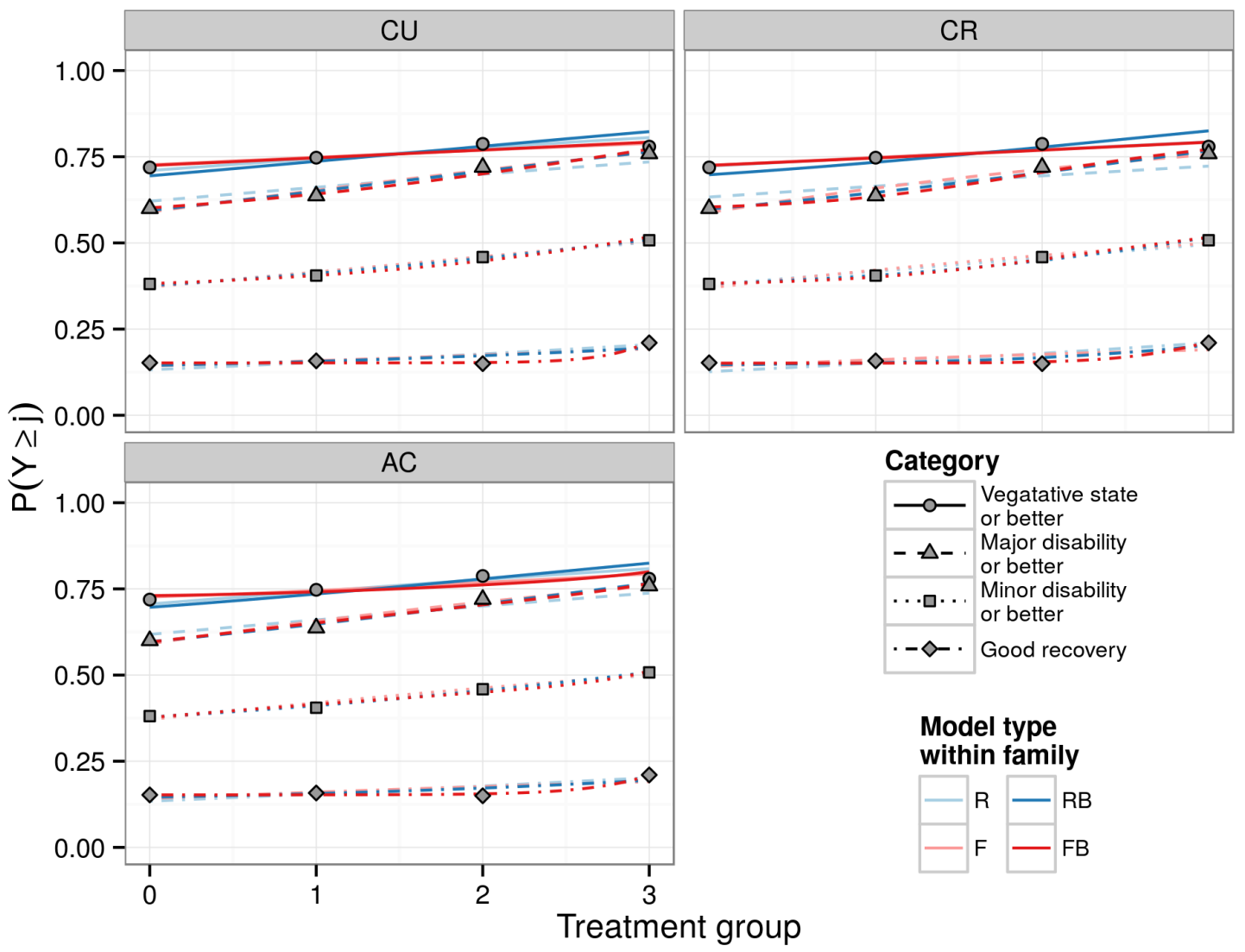

Figure 1.3: Cumulative probability estimates of $\mathrm{CU}, \mathrm{CR}$ and $\mathrm{AC}$ fits to the Glasgow Outcome Scale data. The model fits are represented by lines while the data themselves are displayed as points.

$(0.28,0.12,0.22,0.23,0.15)^{\top}$ obtained from Table 1.1 , and similarly for the other dose types.

To generate a particular simulated data set we used the sample proportions in Table 1.1 as the true $P\left(Y_{i j}=1\right)$ for all $j=1, \ldots, C$ and $i=1, \ldots, n$. These probabilities were used to generate a C-dimensional multinomial indicator vector $\mathbf{y}_{i}$ for each $i=1, \ldots, n$ in which there are $C-1$ components with value zero and a single component with value one. The twelve models presented in Sections 1.2 and 1.4 were 


\begin{tabular}{|c|c|c|c|c|c|c|}
\hline \multirow{2}{*}{ Model } & \multicolumn{2}{|c|}{$\mathrm{CU}$} & \multicolumn{2}{|c|}{$\mathrm{CR}$} & \multicolumn{2}{|c|}{$\mathrm{AC}$} \\
\hline & Reduced & Full & Reduced & Full & Reduced & Full \\
\hline Exposure & 2465.8 & 2454.4 & 2467.0 & 2456.6 & 2465.7 & 2456.7 \\
\hline Background & 2465.5 & 2458.4 & 2465.2 & 2456.9 & 2465.4 & 2461.9 \\
\hline
\end{tabular}

Table 1.4: Mean AIC of the model fits over 1,000 simulated replicates from the Glasgow Outcome Scale data. Results for each of the six models in Section 1.2 are shown in the top row; the results for the corresponding background models are given in the bottom row.

then fit to the data set resulting in an estimate of $\pi_{i j}=P\left(Y_{i j}=1\right)$ for all $j=1, \ldots, C$ for each model. This procedure was repeated for each of 1,000 data sets. Finally, for each model, we compute the log-likelihood and AIC.

We summarize the simulation by plotting the mean fits in Figure 1.4 and give the means of the AIC values in Table 1.4. We also provide the mean residuals in Table 1.5, and note that the standard error for all values in this table is at most 0.003 .

As with the model fits to the original data, Figure 1.4 indicates that there is little to distinguish the various models in the simulation. The mean AIC values for the simulation in Table 1.4 reveal the same pattern as in Table 1.3; within each family the model with separate effects and no background parameter is the best on average, while CUF provides the best fit on average overall. 


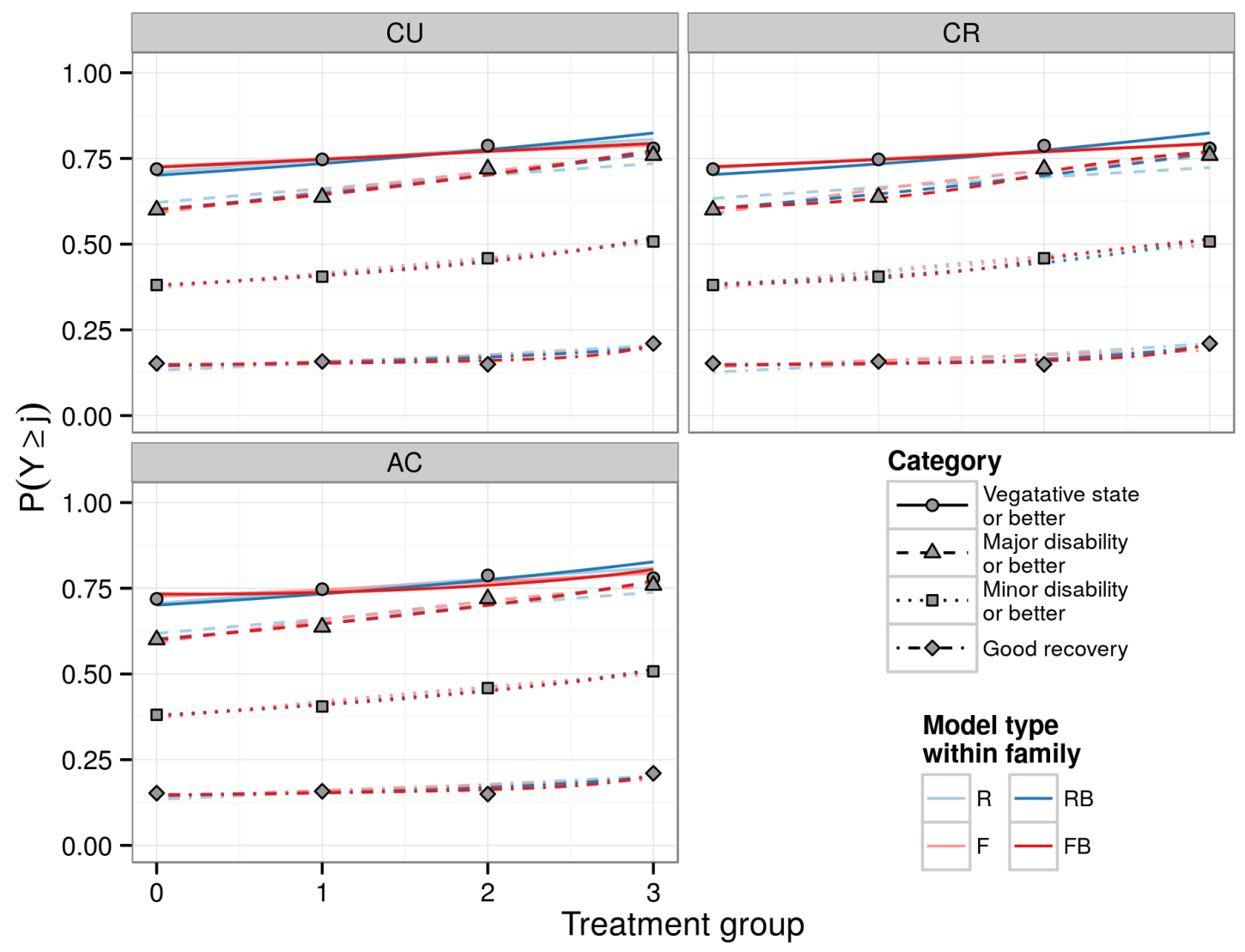

Figure 1.4: Mean cumulative probability estimates of CU, CR and AC fits over 1,000 simulated replicates from the Glasgow Outcome Scale data. The mean model fits are represented by lines while the true cumulative probability of each of the dose levels in the design are displayed as points. 


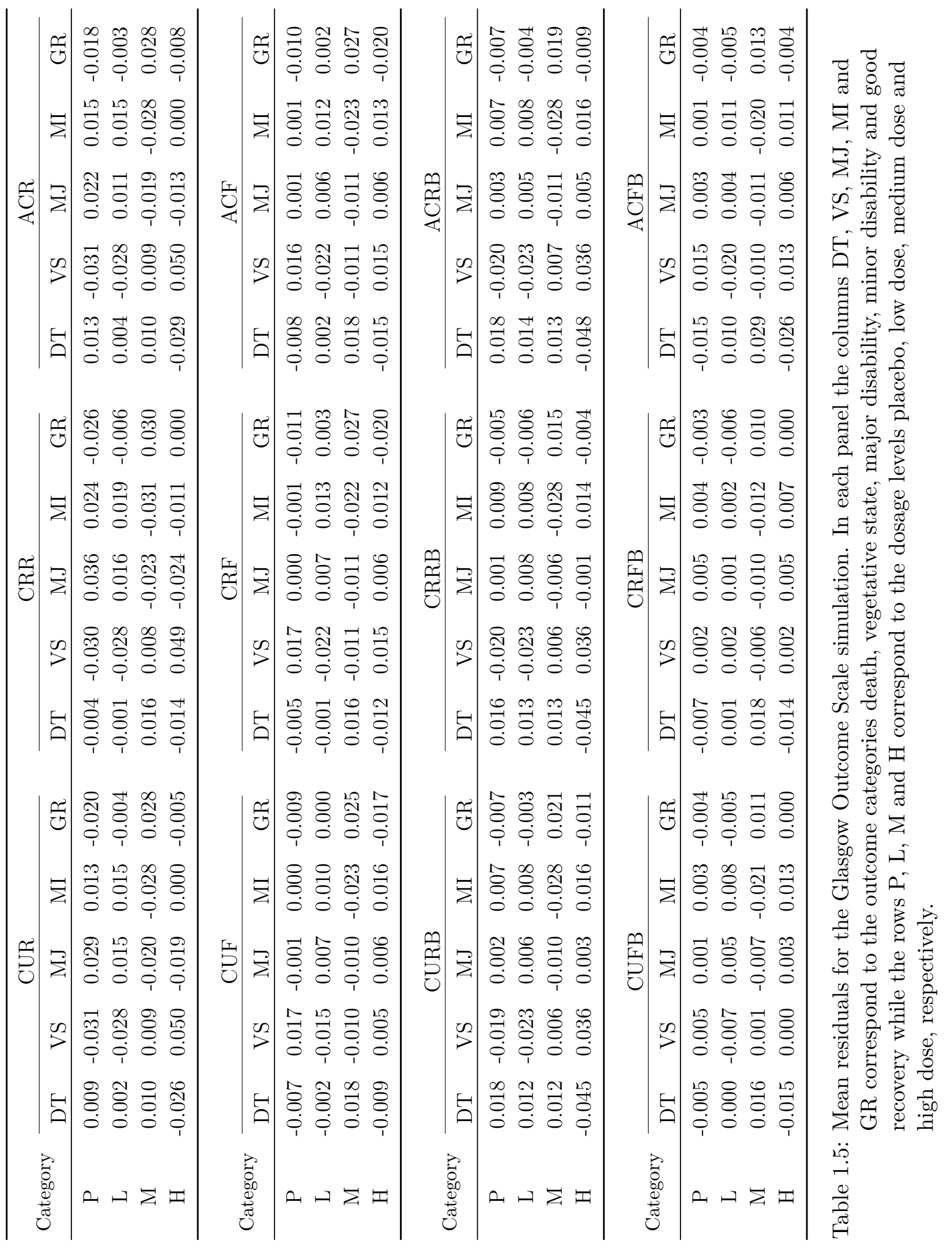




\subsubsection{Tinaroo Virus Data}

We now fit the four cumulative link models to the Tinaroo virus data provided in Table 1.2 using a $\log _{10}$ transformation of the dose. These fits are displayed in Figure 1.5 while the log-likelihood and AIC are shown in Table 1.6.

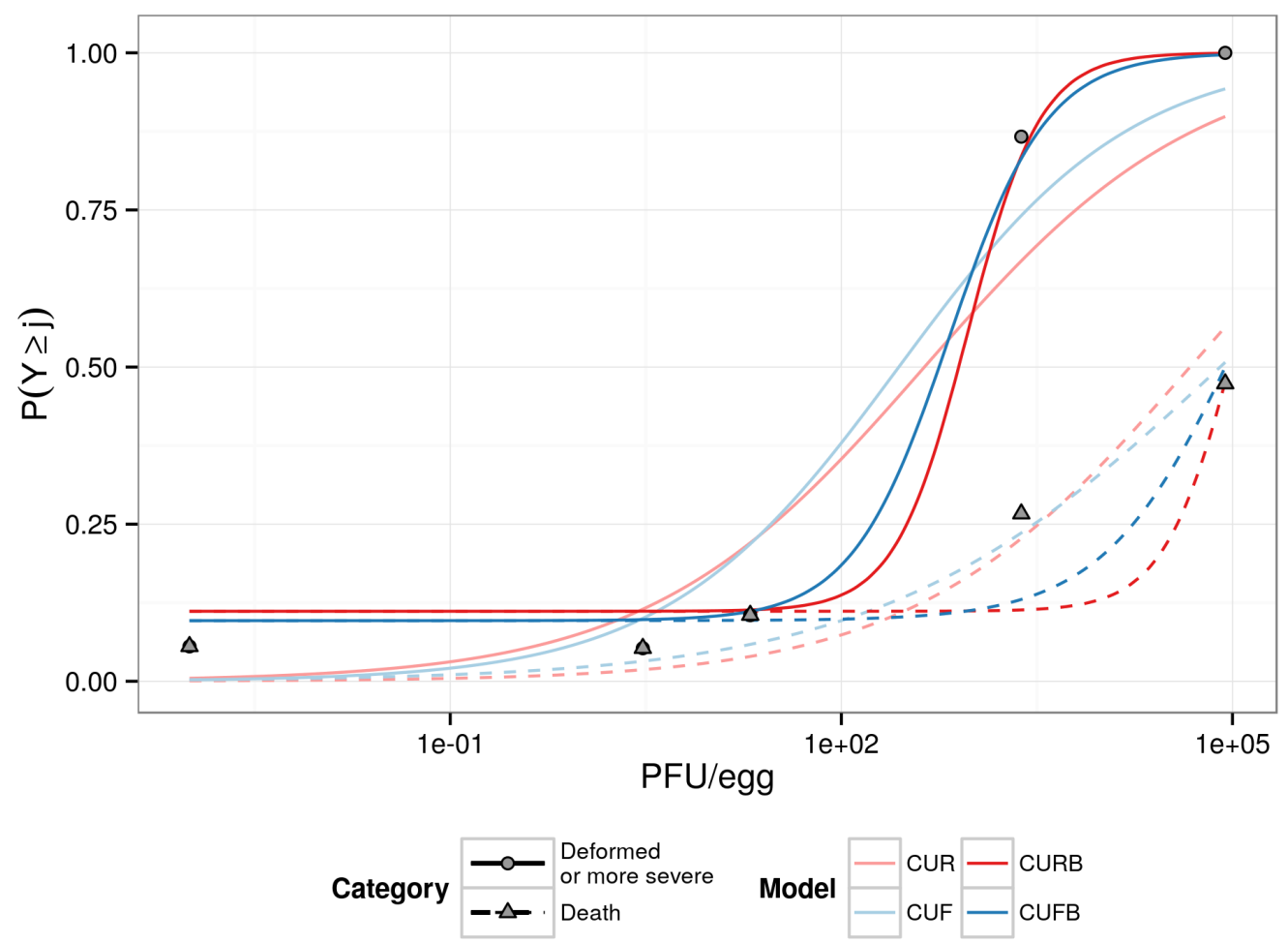

Figure 1.5: Cumulative probability estimates of $\mathrm{CU}$ fits to the Tinaroo virus data. The model fits are represented by lines while the data themselves are displayed as points.

We can see in Table 1.6 that the log-likelihood of CUFB is slightly larger than that of CURB, however the CURB model provides the best fit according to the AIC. Since CURB and CUFB models result in similar fits, but CURB uses less parameters, we will not investigate CUFB further. 


\begin{tabular}{lcccccc}
\hline & \multicolumn{2}{c}{ Exposure } & & \multicolumn{2}{c}{ Background } \\
\cline { 2 - 3 } \cline { 5 - 6 } & CUR & CUF & & CURB & CURF \\
\hline Log-likelihood & -55.8 & -50.4 & & -43.4 & -43.1 \\
AIC & 117.6 & 108.8 & & 96.7 & 98.1 \\
\hline
\end{tabular}

Table 1.6: Log-likelihood and AIC of fits for each of the four CU family models to the Tinaroo virus data.

\begin{tabular}{lccc}
\hline \multirow{2}{*}{$\mathrm{X}$} & \multicolumn{3}{c}{ Category } \\
\cline { 2 - 4 } & 1 & 2 & 3 \\
\hline $\log 0.001$ & 0.889 & 0.000 & 0.111 \\
$\log 3$ & 0.888 & 0.000 & 0.111 \\
$\log 20$ & 0.887 & 0.002 & 0.111 \\
$\log 2,400$ & 0.164 & 0.722 & 0.113 \\
$\log 88,000$ & 0.001 & 0.523 & 0.477 \\
\hline
\end{tabular}

Table 1.7: Estimated response category probabilities from the CURB fit to the Tinaroo virus data.

We now consider a scenario in which the underlying generating mechanism for the data can be modeled by a CURB model. Table 1.7 provides the estimated response category probabilities from the CURB fit to the Tinaroo virus data.

We use these probabilities to generate 1,000 data sets in a similar fashion as the previous simulation. Then, for each data set, we fit the CUR, CUF and CURB models and also compute the log-likelihood and AIC. Figure 1.6 plots the mean fits for the simulation, Table 1.8 gives the mean log-likelihood and AIC, and Table 1.9 contains the mean residuals and their standard deviations. 


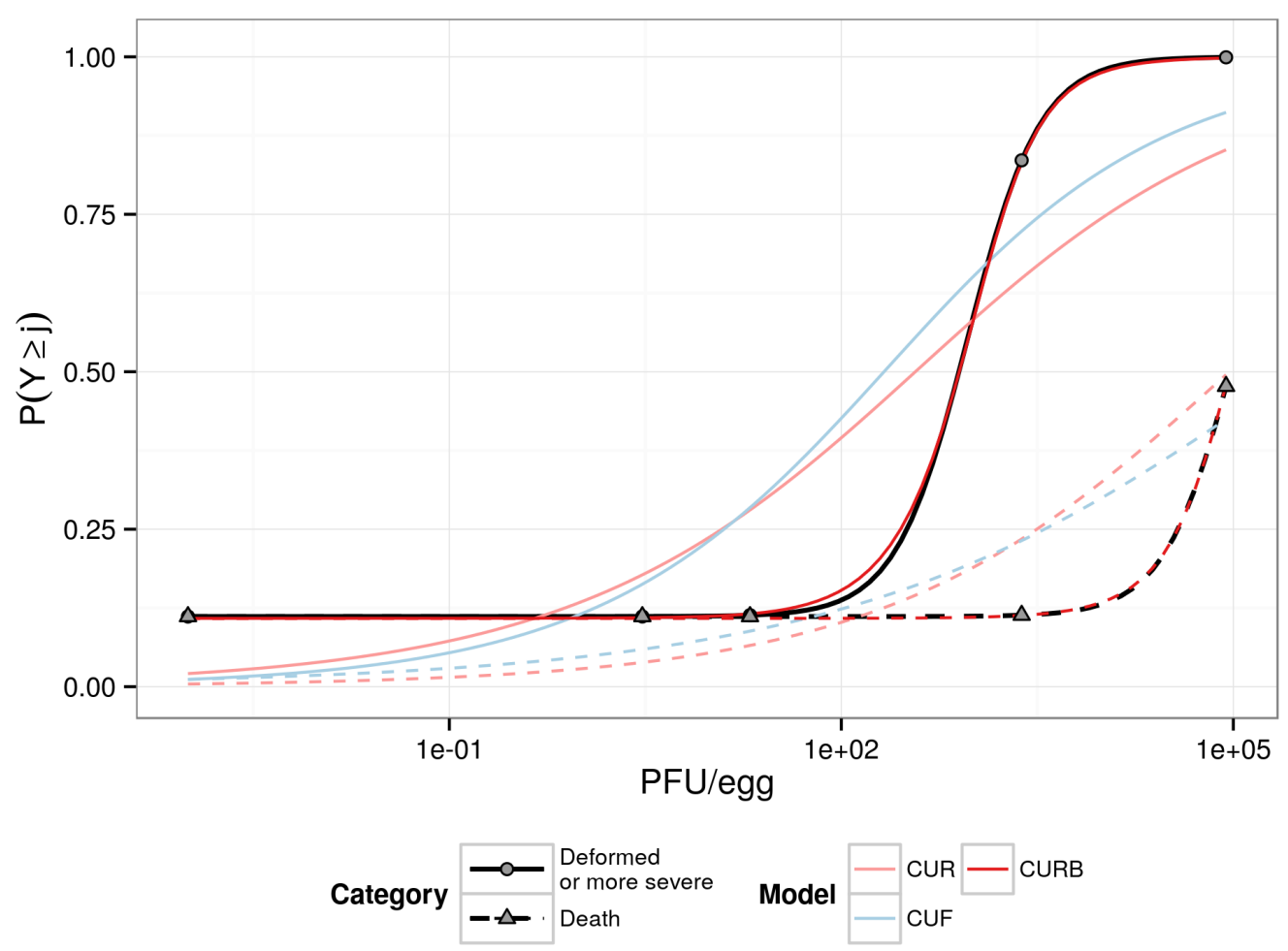

Figure 1.6: Mean cumulative probability estimates of CU fits over 1,000 simulated replicates from the CURB fit to the Tinaroo virus data. The true underlying probabilities of the generated data are given in black and the dosage levels used in the simulation are indicated by points.

\begin{tabular}{lcccc}
\hline & \multicolumn{2}{c}{ Exposure } & & Background \\
\cline { 2 - 3 } \cline { 5 - 5 } \cline { 5 - 5 } Mean Loglikelihood & -127.5 & -113.4 & & -88.2 \\
Mean AIC & 261.0 & 234.9 & & 186.5 \\
\hline
\end{tabular}

Table 1.8: Mean log-likelihood and mean AIC for the simulation based on the Tinaroo virus data. 
Both the mean fits in Figure 1.6 and the mean AICs in Table 1.8 show that the CURB model outperforms CUR and CUF in the simulation. Figure 1.6 also shows the inability of CUR and CUF to adequately track the data when there is a non-zero background response at low dose levels. Finally, we can see that the residuals of CURB in Table 1.9 have little bias, whereas CUR and CUF are strongly biased.

\begin{tabular}{|c|c|c|c|c|c|c|c|c|c|}
\hline \multirow{3}{*}{ X } & \multicolumn{6}{|c|}{ Exposure } & \multirow{2}{*}{\multicolumn{3}{|c|}{$\begin{array}{c}\text { Background } \\
\text { CURB }\end{array}$}} \\
\hline & \multicolumn{3}{|c|}{ CUR } & \multicolumn{3}{|c|}{ CUF } & & & \\
\hline & $j=1$ & $j=2$ & $j=3$ & $j=1$ & $j=2$ & $j=3$ & $j=1$ & $j=2$ & $j=3$ \\
\hline \multirow[t]{2}{*}{$\log 0.001$} & 0.091 & 0.017 & -0.107 & 0.100 & 0.000 & -0.100 & 0.003 & 0.000 & -0.003 \\
\hline & $(0.02)$ & $(0.01)$ & $(0.00)$ & $(0.01)$ & $(0.00)$ & $(0.01)$ & $(0.03)$ & $(0.00)$ & $(0.03)$ \\
\hline \multirow[t]{2}{*}{$\log 3$} & -0.066 & 0.139 & -0.073 & -0.051 & 0.103 & -0.052 & 0.002 & 0.001 & -0.003 \\
\hline & $(0.05)$ & $(0.03)$ & $(0.02)$ & $(0.05)$ & $(0.03)$ & $(0.03)$ & $(0.03)$ & $(0.01)$ & $(0.03)$ \\
\hline \multirow[t]{2}{*}{$\log 20$} & -0.167 & 0.213 & -0.046 & -0.171 & 0.194 & -0.023 & -0.002 & 0.005 & -0.003 \\
\hline & $(0.05)$ & $(0.03)$ & $(0.03)$ & $(0.06)$ & $(0.03)$ & $(0.03)$ & $(0.03)$ & $(0.01)$ & $(0.03)$ \\
\hline \multirow[t]{2}{*}{$\log 2,400$} & 0.187 & -0.308 & 0.121 & 0.113 & -0.231 & 0.118 & 0.003 & -0.003 & 0.000 \\
\hline & $(0.03)$ & $(0.06)$ & $(0.05)$ & $(0.04)$ & $(0.06)$ & $(0.04)$ & $(0.07)$ & $(0.07)$ & $(0.03)$ \\
\hline \multirow[t]{2}{*}{$\log 88,000$} & 0.147 & -0.165 & 0.018 & 0.088 & -0.036 & -0.052 & 0.001 & -0.004 & 0.003 \\
\hline & $(0.04)$ & $(0.05)$ & $(0.06)$ & $(0.03)$ & $(0.06)$ & $(0.07)$ & $(0.01)$ & $(0.08)$ & $(0.08)$ \\
\hline
\end{tabular}

Table 1.9: Mean residuals and standard deviations (below in brackets) for each category $(j=1,2,3)$ for the simulation based on the Tinaroo virus data. 


\begin{tabular}{lccccc}
\hline & \multicolumn{5}{c}{ Parameter } \\
\cline { 2 - 6 } & $\zeta_{1}$ & $\zeta_{2}$ & $\alpha_{1}$ & $\alpha_{2}$ & $\beta_{1}$ \\
\hline Mean & 0.000 & 0.108 & 11.457 & 19.427 & -1.675 \\
Sample Standard Deviation & $(0.00)$ & $(0.03)$ & $(3.42)$ & $(5.17)$ & $(0.45)$ \\
\hline Truth & 0.000 & 0.111 & 10.908 & 18.487 & -1.592 \\
\hline
\end{tabular}

Table 1.10: Mean estimates and sample standard deviations of the parameters for the CURB model for the simulation based on the Tinaroo virus data. The final row gives the parameter values of the CURB model fit to the Tinaroo virus data.

As a final illustration of the performance of the CURB model for describing the Tinaroo virus data, in Table 1.10 we provide the average parameter estimates of the CURB model and the parameter values used to generate the simulated data sets. The average parameter estimates appear to have small bias.

\subsection{Conclusion and Discussion}

We have presented a summary of the existing dose-response models for ordinal outcome data. Generally speaking, these models can be distinguished by the form of $\gamma_{j}(\mathbf{x})$; these include cumulative, continuation-ratio, or adjacent category families. A general extension to ordinal models has been provided here which accommodates non-zero probabilities of response for the control group by incorporating a background response parameter vector. We illustrate via a simulation study the difficulties that can be encountered in model fitting when significant background responses are not acknowledged. Nevertheless, there will be dose response curves for ordinal outcome data that, as a result of their shape, simply cannot be described well by the existing 
CHAPTER 1. ORDINAL MODELS AND A BACKGROUND PARAMETER 40

models, either with or without an acknowledgement of background response. One possibility for dealing with such situations might be to use a model based on splines that can better adjust to the shape of the dose response curve. We explore this in the next chapter. 


\section{Chapter 2}

\section{Modelling Ordinal Data with Splines}

\section{$2.1 \quad$ Introduction}

For ordinal data, the cumulative logit model $(\mathrm{CU})$, is used most often in the literature, both in general and Epidemiology specifically. There are a number of other possibilities, two of which are the continuous ratio model (CR) and adjacent cate-

gories model $(\mathrm{AC})$ which were introduced in Section 1.2. In many situations these parametric models are not able to track the underlying true response curve of interest. In this chapter we propose a flexible model for fitting smooth monotone functions to ordinal responses, and discuss two methods of estimating the model.

In Section 2.2 we present some required statistical background material for the model we propose in Section 2.3. Also in Section 2.3, we discuss two methods of estimating this model; the first is an adaptive fixed knot approach and the second is penalty-based. We fit the model via both approaches to a data set in Section 2.4 and compare the behaviour of the resulting fits to those obtained from a CUR model. In Section 2.5 we conduct a number of simulations to investigate the properties of the model. Finally, we give a conclusion in Section 2.6. 


\section{$2.2 \quad$ Background}

In this section we provide some background material prior to the introduction of the monotone smoothing models in Section 2.3.

\subsubsection{Spline Smoothing}

Assume that a function $f$ is an unknown smooth function. We can estimate $f$ using splines as follows

$$
f(u)=\boldsymbol{\Psi}(u) \boldsymbol{\beta}
$$

where $\boldsymbol{\Psi}(u)=\left(\Psi_{1}(u), \ldots, \Psi_{K}(u)\right)$ is a row vector of known basis functions evaluated at $u$ which span the vector space of interest.

We let the ordered set $\left\{t_{1}, \ldots, t_{K}\right\}$ where $t_{1}<\cdots<t_{K}$ and $t_{k} \in \mathcal{D}$, represent the knot locations. We refer to the first and last knots, $t_{1}$ and $t_{K}$ respectively, as the boundary knots and the others as the interior knots. Several basis functions are in common use; we present the recursive definition of the B-spline basis over a domain $\mathcal{D}$ below (see de Boor, 1978). An attractive property of the B-spline basis is its numerical stability and computational efficiency.

The $k$-th B-spline basis function of degree $l$ is defined as $\Psi_{k}^{l}(u)$, where

$$
\Psi_{k}^{0}(u)=\left\{\begin{array}{cc}
1, & t_{k} \leq u<t_{k+1} \\
0, & \text { otherwise }
\end{array}\right.
$$


and

$$
\Psi_{k}^{l}(u)=\frac{u-t_{k}}{t_{k+l}-t_{k}} \Psi_{k}^{l-1}(u)+\frac{t_{k+l+1}-u}{t_{k+l+1}-t_{k+1}} \Psi_{k+1}^{l-1}(u)
$$

recursively for $l=1, \ldots$.

\subsubsection{Monotonic Spline Smoothing}

There are certain circumstances, such as the dose-response context, where it may be desirable and warranted to restrict a function to be monotone. One method of enforcing such a condition is with a spline constructed with a B-spline basis; constraining the spline coefficient to be ordered is sufficient to impose monotonicity. However, for splines of order 4 or higher, ordered coefficients is not a necessary condition for monotonicity. Nonetheless, this is a popular approach to the problem (see, among others, Kelly and Rice, 1990; Kong and Eubank, 2006; Lu et al., 2009; Lu and Chiang, 2011). An alternative formulation is offered by way of integrated splines (I-splines) of Ramsay (1988). The I-spline approach is equivalent to ordered coefficients, however monotonicity is enforced with a positivity constraint. These methods are prone to accruing bias as they impose strong monotonicity which implies that $f(x)<f(x+\epsilon)$ for any $\epsilon>0$.

As an alternative to ordered coefficients and I-splines for imposing monotonicity, we can consider constructing a monotone smooth function by integrating a function which is positive, see Ramsay and Silverman (2005). For example, the function

$$
\int_{t_{1}}^{x} \exp (\boldsymbol{\Psi}(u) \boldsymbol{\beta}) \mathrm{du}
$$


which is weakly monotonically increasing in $x$. This approach allows us to fit weakly monotone functions instead of the stong conditions of the previous approach (i.e. $f(x) \leq f(x+\epsilon))$. As such it mitigates the upward bias problems that occur in the previously discussed approaches baring imposing non-linear constraints when maximizing the likelihood function. However, in general this occurs at the expense of requiring numerical integration also referred to as quadrature.

\subsubsection{Ordinal Modeling and Estimation}

Recall from Section 1.3 that we expressed an observation from a $C$-category ordinal random variable by a multinomial indicator vector of length $C$. Note however that one element of such a vector is redundant and a vector indicating the outcomes of the first $C-1$ categories is sufficient.

Here, we represent a $C$-category ordinal random variable by $\mathbf{Y}^{\star}=\left(Y_{1}, \ldots, Y_{C-1}\right)^{\top}$, where the random variables $Y_{j}$ can take on values 0 or 1 and $\sum_{j=1}^{C-1} Y_{j} \leq 1$. In an analogous manner, we represent an observation from $\mathbf{Y}^{\star}$ by $\mathbf{y}^{\star}=\left(y_{1}, \ldots, y_{C-1}\right)^{\top}$. If $\pi_{j}=P\left(Y_{j}=1\right)$ and $\pi_{C}=1-\sum_{j=1}^{C-1} \pi_{j}$ then $\mathbf{Y}^{\star} \sim \operatorname{Multinomial}(1, \boldsymbol{\pi})$, with parameter $\boldsymbol{\pi}=\left(\pi_{1}, \ldots, \pi_{C}\right)^{\top}$. For convenience we also define $\boldsymbol{\pi}^{\star}=\left(\pi_{1}, \ldots, \pi_{C-1}\right)^{\boldsymbol{\top}}$ and 
$y_{C}=1-\sum_{j=1}^{C-1} y_{j}$. The probability mass function of $\mathbf{Y}^{\star}$ is then

$$
\begin{aligned}
f\left(\mathbf{y}^{\star} ; \boldsymbol{\theta}\right)=\prod_{j=1}^{C} \pi_{j}^{y_{j}} & =\exp \left\{\sum_{j=1}^{C} y_{j} \log \pi_{j}\right\} \\
& =\exp \left\{\sum_{j=1}^{C-1} y_{j} \log \pi_{j}+\left(1-\sum_{j=1}^{C-1} y_{j}\right) \log \pi_{C}\right\} \\
& =\exp \left\{\sum_{j=1}^{C-1} y_{j} \log \left(\frac{\pi_{j}}{\pi_{C}}\right)+\log \pi_{C}\right\} \\
& =\exp \left\{\sum_{j=1}^{C-1} y_{j} \phi_{j}-b(\boldsymbol{\phi})\right\} \\
& =\exp \left\{\mathbf{y}^{\star \top} \boldsymbol{\phi}-b(\boldsymbol{\phi})\right\}
\end{aligned}
$$

where $\phi_{j}=\log \left(\frac{\pi_{j}}{\pi_{C}}\right)$ are the elements of $\phi=\left(\phi_{1}, \ldots, \phi_{C-1}\right)^{\top}$ and the function $b(\phi)=\log \left\{1+\sum_{j=1}^{C-1} \exp \left(\phi_{j}\right)\right\}$ depends only on $\phi$. Observe that the last expression in Equation (2.3) is written as a (C-1)-parameter exponential family, thus we have $\mathrm{E}\left(\mathbf{Y}^{\star}\right)=\boldsymbol{\pi}^{\star}=\frac{\partial}{\partial \phi^{\top}} b(\boldsymbol{\phi})$ and $\operatorname{Cov}\left(\mathbf{Y}^{\star}\right)=\frac{\partial^{2}}{\partial \phi \partial \phi^{\top}} b(\boldsymbol{\phi})=\operatorname{diag}\left\{\boldsymbol{\pi}^{\star}\right\}-\boldsymbol{\pi}^{\star} \boldsymbol{\pi}^{\star \top}$.

\section{Maximum Likelihood Estimation}

Now, suppose that $\mathbf{Y}_{i}^{\star}=\left(Y_{i 1}, \ldots, Y_{i, C-1}\right)^{\top}$ are independent $C$-category ordinal random variables, $i=1, \ldots, n$. In addition, suppose that the response probabilities for category $j, \pi_{i j}(\boldsymbol{\theta})=P\left(Y_{i j}=1 \mid \mathbf{x}_{i}\right)$, are a function of some vector $\mathbf{x}_{i}$ which is specific to individual $i$, and also of a vector $\boldsymbol{\theta}$ which is common to all individuals.

Similarly to above, we define the vectors $\boldsymbol{\pi}_{i}^{\star}(\boldsymbol{\theta})=\left(\pi_{i 1}(\boldsymbol{\theta}), \ldots, \pi_{i, C-1}(\boldsymbol{\theta})\right)^{\top}$ and $\phi_{i}(\boldsymbol{\theta})=\left(\phi_{i 1}(\boldsymbol{\theta}), \ldots, \phi_{i, C-1}(\boldsymbol{\theta})\right)^{\top}$, where $\phi_{i j}(\boldsymbol{\theta})=\log \left(\frac{\pi_{i j}(\boldsymbol{\theta})}{\pi_{i C}(\boldsymbol{\theta})}\right), j=1, \ldots, C-1$. We also have that $\mathrm{E}\left(\mathbf{Y}_{i}^{\star}\right)=\boldsymbol{\pi}_{i}^{\star}(\boldsymbol{\theta})$ and $\operatorname{Cov}\left(\mathbf{Y}_{i}^{\star}\right)=\operatorname{diag}\left\{\boldsymbol{\pi}_{i}^{\star}(\boldsymbol{\theta})\right\}-\boldsymbol{\pi}_{i}^{\star}(\boldsymbol{\theta}) \boldsymbol{\pi}_{i}^{\star}(\boldsymbol{\theta})^{\top}$ are the 
expected value and the covariance matrix of $\mathbf{Y}_{i}^{\star}$ respectively. We can write this expectation as $\mathrm{E}\left(\mathbf{Y}_{i}^{\star}\right)=\frac{\partial}{\partial \phi^{\top}{ }_{i}} b\left(\boldsymbol{\phi}_{i}(\boldsymbol{\theta})\right)$ and the covariance as $\operatorname{Cov}\left(\mathbf{Y}_{i}^{\star}\right)=\frac{\partial^{2}}{\partial \phi_{i} \partial \phi_{i}^{\top}} b\left(\boldsymbol{\phi}_{i}(\boldsymbol{\theta})\right)$, hence

$$
\frac{\partial \boldsymbol{\pi}_{i}^{\star}(\boldsymbol{\theta})}{\partial \boldsymbol{\theta}}=\frac{\partial}{\partial \boldsymbol{\theta}}\left(\frac{\partial b\left(\boldsymbol{\phi}_{i}(\boldsymbol{\theta})\right)}{\partial \boldsymbol{\phi}_{i}^{\top}{ }_{i}}\right)=\frac{\partial^{2} b\left(\boldsymbol{\phi}_{i}\right)}{\partial \boldsymbol{\phi}_{i} \partial \boldsymbol{\phi}_{i}^{\top}} \frac{\partial \boldsymbol{\phi}_{i}(\boldsymbol{\theta})}{\partial \boldsymbol{\theta}}=\operatorname{Cov}\left(\mathbf{Y}_{i}^{\star}\right) \frac{\partial \boldsymbol{\phi}_{i}(\boldsymbol{\theta})}{\partial \boldsymbol{\theta}}
$$

Thus, if we let $\mathbf{Z}_{i}(\boldsymbol{\theta})=\frac{\partial \boldsymbol{\pi}_{i}^{\star}(\boldsymbol{\theta})}{\partial \boldsymbol{\theta}}$ and denote the covariance matrix by $\mathbf{V}_{i}^{\star}(\boldsymbol{\theta})$ then $\frac{\partial \phi_{i}(\boldsymbol{\theta})}{\partial \boldsymbol{\theta}}=\mathbf{V}_{i}^{\star}(\boldsymbol{\theta})^{-1} \mathbf{Z}_{i}(\boldsymbol{\theta})$.

We now consider the log-likelihood of the data $\left\{\mathbf{y}_{1}^{\star}, \ldots, \mathbf{y}_{n}^{\star}\right\}$,

$$
l(\boldsymbol{\theta})=\sum_{i=1}^{n}\left\{\mathbf{y}_{i}^{\star \top} \boldsymbol{\phi}_{i}(\boldsymbol{\theta})-b\left(\boldsymbol{\phi}_{i}(\boldsymbol{\theta})\right)\right\}
$$

and have that

$$
\frac{\partial l(\boldsymbol{\theta})}{\partial \boldsymbol{\theta}}=\sum_{i=1}^{n}\left(\mathbf{y}_{i}^{\star}-\boldsymbol{\pi}_{i}^{\star}(\boldsymbol{\theta})\right)^{\boldsymbol{\top}} \frac{\partial \boldsymbol{\phi}_{i}(\boldsymbol{\theta})}{\partial \boldsymbol{\theta}}=\sum_{i=1}^{n}\left(\mathbf{y}_{i}^{\star}-\boldsymbol{\pi}_{i}^{\star}(\boldsymbol{\theta})\right)^{\top} \mathbf{V}_{i}^{\star}(\boldsymbol{\theta})^{-1} \mathbf{Z}_{i}(\boldsymbol{\theta})
$$

and

$$
\frac{\partial^{2} l(\boldsymbol{\theta})}{\partial \boldsymbol{\theta} \partial \boldsymbol{\theta}^{\boldsymbol{\top}}}=\sum_{i=1}^{n}\left\{-\mathbf{Z}_{i}(\boldsymbol{\theta})^{\top} \mathbf{V}_{i}^{\star}(\boldsymbol{\theta})^{-1} \mathbf{Z}_{i}(\boldsymbol{\theta})+\sum_{j=1}^{C-1}\left(y_{i j}-\pi_{i j}(\boldsymbol{\theta})\right) \frac{\partial^{2} \phi_{i j}(\boldsymbol{\theta})}{\partial \boldsymbol{\theta} \partial \boldsymbol{\theta}^{\boldsymbol{\top}}}\right\}
$$

are the gradient and Hessian of $l(\boldsymbol{\theta})$. Since $\mathrm{E}\left(y_{i j}\right)=\pi_{i j}(\boldsymbol{\theta})$, the terms in the inner summation in (2.6) have an expected value of zero; thus we have the expected Fisher Information matrix $\mathcal{J}(\boldsymbol{\theta})=\mathrm{E}\left(-\frac{\partial^{2} l(\boldsymbol{\theta})}{\partial \boldsymbol{\theta} \partial \boldsymbol{\theta}^{\top}}\right)=\sum_{i=1}^{n} \mathbf{Z}_{i}(\boldsymbol{\theta})^{\top} \mathbf{V}_{i}^{\star}(\boldsymbol{\theta})^{-1} \mathbf{Z}_{i}(\boldsymbol{\theta})$.

Finally, we represent the full data by the vector $\mathbf{y}^{\star \star}=\left(\mathbf{y}_{1}^{\star \top}, \ldots, \mathbf{y}_{n}^{\star \top}\right)^{\top}$, the corresponding vector of random variables by $\mathbf{Y}^{\star \star}=\left(\mathbf{Y}_{1}^{\star \top}, \ldots, \mathbf{Y}_{n}^{\star \top}\right)^{\top}$, the vector of probabilities by $\boldsymbol{\pi}^{\star \star}(\boldsymbol{\theta})=\left(\boldsymbol{\pi}_{1}^{\star}(\boldsymbol{\theta})^{\top}, \ldots, \boldsymbol{\pi}_{n}^{\star}(\boldsymbol{\theta})^{\top}\right)^{\top}$ and define the block diagonal matrices 
$\mathbf{Z}(\boldsymbol{\theta})=\operatorname{blkdiag}\left\{\mathbf{Z}_{1}(\boldsymbol{\theta}), \ldots, \mathbf{Z}_{n}(\boldsymbol{\theta})\right\}$ and $\mathbf{V}^{\star \star}(\boldsymbol{\theta})=\operatorname{blkdiag}\left\{\mathbf{V}_{1}^{\star}(\boldsymbol{\theta}), \ldots, \mathbf{V}_{n}^{\star}(\boldsymbol{\theta})\right\}$. The block diagonal structure of $\mathbf{V}^{\star \star}(\boldsymbol{\theta})$ implies that its inverse is also a block diagonal matrix: $\mathbf{V}^{\star \star}(\boldsymbol{\theta})^{-1}=\operatorname{blkdiag}\left\{\mathbf{V}_{1}^{\star}(\boldsymbol{\theta})^{-1}, \ldots, \mathbf{V}_{n}^{\star}(\boldsymbol{\theta})^{-1}\right\}$. Also, note that since $\mathbf{Y}_{i}^{\star}$, $i=1, \ldots, n$, are independent, we have $\operatorname{Cov}\left(\mathbf{Y}^{\star \star}\right)=\mathbf{V}^{\star \star}(\boldsymbol{\theta})$.

We now have the transpose of the score equation (2.5) as

$$
\frac{\partial l(\boldsymbol{\theta})}{\partial \boldsymbol{\theta}^{\top}}=\mathbf{Z}(\boldsymbol{\theta})^{\top} \mathbf{V}^{\star \star}(\boldsymbol{\theta})^{-1}\left(\mathbf{y}^{\star \star}-\boldsymbol{\pi}^{\star \star}(\boldsymbol{\theta})\right)
$$

and the expected Fisher Information matrix as

$$
\mathcal{J}(\boldsymbol{\theta})=\mathbf{Z}(\boldsymbol{\theta})^{\top} \mathbf{V}^{\star \star}(\boldsymbol{\theta})^{-1} \mathbf{Z}(\boldsymbol{\theta}) .
$$

\section{Fisher Scoring}

One iterative approach to computing the maximum likelihood estimator is the Fisher Scoring method. If $\boldsymbol{\theta}_{[t]}$ is the $t$-th update of $\boldsymbol{\theta}$ then the Fisher scoring update is

$$
\begin{aligned}
\boldsymbol{\theta}_{[t+1]} & =\boldsymbol{\theta}_{[t]}+\mathcal{J}\left(\boldsymbol{\theta}_{[t]}\right)^{-1} \frac{\partial l\left(\boldsymbol{\theta}_{[t]}\right)}{\partial \boldsymbol{\theta}^{\top}} \\
& =\boldsymbol{\theta}_{[t]}+\left[\mathbf{Z}\left(\boldsymbol{\theta}_{[t]}\right)^{\top} \mathbf{V}^{\star \star}\left(\boldsymbol{\theta}_{[t]}\right)^{-1} \mathbf{Z}\left(\boldsymbol{\theta}_{[t]}\right)\right]^{-1} \mathbf{Z}\left(\boldsymbol{\theta}_{[t]}\right)^{\top} \mathbf{V}^{\star \star}\left(\boldsymbol{\theta}_{[t]}\right)^{-1}\left[\mathbf{y}^{\star \star}-\boldsymbol{\pi}^{\star \star}\left(\boldsymbol{\theta}_{[t]}\right)\right] .
\end{aligned}
$$

If we let $\widetilde{\mathbf{y}}^{\star \star}\left(\boldsymbol{\theta}_{[t]}\right)=\mathbf{Z}\left(\boldsymbol{\theta}_{[t]}\right) \boldsymbol{\theta}_{[t]}+\mathbf{y}^{\star \star}-\boldsymbol{\pi}^{\star \star}\left(\boldsymbol{\theta}_{[t]}\right)$ be the quasi-data we can also write the update as

$$
\boldsymbol{\theta}_{[t+1]}=\left[\mathbf{Z}\left(\boldsymbol{\theta}_{[t]}\right)^{\top} \mathbf{V}^{\star \star}\left(\boldsymbol{\theta}_{[t]}\right)^{-1} \mathbf{Z}\left(\boldsymbol{\theta}_{[t]}\right)\right]^{-1} \mathbf{Z}\left(\boldsymbol{\theta}_{[t]}\right)^{\top} \mathbf{V}^{\star \star}\left(\boldsymbol{\theta}_{[t]}\right)^{-1} \widetilde{\mathbf{y}}^{\star \star}\left(\boldsymbol{\theta}_{[t]}\right)
$$

Thus the Fisher scoring update is the same as an iteratively reweighted least squares problem with weights given by $\mathbf{V}^{\star \star}\left(\boldsymbol{\theta}_{[t]}\right)^{-1}$. 


\section{Multivariate Generalized Additive Model}

Recall from the previous chapter that the CUR model (given in Equation (1.3)) is a multivariate GLM and that it is also a subclass of the model in (1.1). The CUR model with a logit link is commonly referred to as the cumulative logit link model and can be written as

$$
\operatorname{logit}[P(Y \leq j \mid \mathbf{x})]=\alpha_{j}+\boldsymbol{\beta}^{\top} \mathbf{x}, \quad j=1, \ldots, C-1
$$

where $\mathbf{x}$ is a vector of covariates associated with $Y$.

Hastie and Tibshirani (1987) discuss the more general model

$$
\operatorname{logit}[P(Y \leq j \mid \mathbf{x})]=\alpha_{j}+\sum_{s=1}^{r} f_{s}\left(x_{s}\right), \quad j=1, \ldots, C-1
$$

where the $f_{s}\left(x_{s}\right)$ are arbitrary functions and $x_{s}$ is the $s$-th component of $\mathbf{x}$. This model is an instance of a multivariate GAM, which are examined in-depth in the book Hastie and Tibshirani (1990). The model allows for each covariate $x_{s}$ to have a non-linear relationship to the log odds of the cumulative probabilities. Using splines as in (2.1) for the smooth functions in (2.11), gives the model

$$
\operatorname{logit}[P(Y \leq j \mid \mathbf{x})]=\alpha_{j}+\sum_{s=1}^{r} \boldsymbol{\Psi}_{s}\left(x_{s}\right) \boldsymbol{\beta}_{s}, \quad j=1, \ldots, C-1
$$

A semi-parametric model is obtained when some of the $f_{s}\left(x_{s}\right)=x_{s} \beta_{s}$ are linear; if all $f_{s}\left(x_{s}\right)$ are linear then (2.11) reduces to the fully-parametric CUR model in (2.10).

In Section 1.3 we present a derivation of the loglikelihood which allows one to estimate the model (1.1) via the MLE method; in this section we write the likelihood so that we can exploit some of the characteristics which result from using the canonical 
logit link and the CU family.

Recall from Section 1.3.1 that the CU family defines a linear transformation between $\boldsymbol{\pi}_{i}$ and $\boldsymbol{\gamma}_{i}$ and that $\mathbf{M}$ is a $C-1 \times C-1$ lower triangular matrix. We have that $\boldsymbol{\gamma}_{i}=\mathbf{M} \boldsymbol{\pi}_{i}^{\star}$ and $\boldsymbol{\pi}_{i}^{\star}=\mathrm{M}^{-1} \boldsymbol{\gamma}_{i}$ where

$$
\mathbf{M}^{-1}=\left[\begin{array}{cccc}
1 & & & 0 \\
-1 & 1 & & \\
& \ddots & \ddots & \\
0 & & -1 & 1
\end{array}\right]
$$

is the inverse of $\mathbf{M}$. Now, noting that the first derivative of the inverse logit link function, $g^{-1}=\operatorname{logit}^{-1}$, is $\left[g^{-1}\right]^{(1)}=g^{-1}\left(1-g^{-1}\right)$, we have

$$
\mathbf{E}_{i}=\frac{\partial \boldsymbol{\gamma}_{i}}{\partial \boldsymbol{\eta}_{i}}=\frac{\partial \mathbf{g}\left(\boldsymbol{\eta}_{i}\right)^{-1}}{\partial \boldsymbol{\eta}_{i}}=\operatorname{diag}\left\{\boldsymbol{\gamma}_{i}\left(1-\boldsymbol{\gamma}_{i}\right)\right\}
$$

as defined in (1.18). Combined, we have

$$
\mathbf{Z}_{i}(\boldsymbol{\theta})=\frac{\partial \boldsymbol{\pi}_{i}^{\star}}{\partial \boldsymbol{\theta}}=\mathbf{M}^{-1} \frac{\partial \boldsymbol{\gamma}_{i}}{\partial \boldsymbol{\eta}_{i}} \frac{\partial \boldsymbol{\eta}_{i}}{\partial \boldsymbol{\theta}}=\mathbf{M}^{-1} \operatorname{diag}\left\{\boldsymbol{\gamma}_{i}\left(1-\boldsymbol{\gamma}_{i}\right)\right\} \frac{\partial \boldsymbol{\eta}_{i}}{\partial \boldsymbol{\theta}}
$$

The logistic CUR model in (2.10) and the GAM in (2.12) both have a linear form

for $\boldsymbol{\eta}_{i}$. Thus, $\frac{\partial \boldsymbol{\eta}_{i}}{\partial \boldsymbol{\theta}}$ is not a function of $\boldsymbol{\theta}$, and so if these models are estimated via the Fisher Scoring method described above, this matrix does not need to be recalculated at each iteration.

\subsubsection{Properties of Estimators}

In this section we give some statistical properties for estimators in general. 


\section{Consistency and Bias of Estimators}

The bias of an estimator can be expressed as

$$
\text { Bias in } \hat{\theta}=\mathrm{E}(\hat{\theta})-\theta
$$

and an estimator is said to be unbiased if $E(\hat{\theta})=\theta$. An estimator is said to be consistent if $\hat{\theta} \stackrel{p}{\rightarrow} \theta$, that is if $\hat{\theta}$ converges in probability to $\theta$.

\section{Asymptotic Distribution of Maximum Likelihood Estimators}

Under mild regularity conditions, the maximum likelihood estimator $\hat{\boldsymbol{\theta}}$ is consistent and has the asymptotic normal distribution $N\left(\boldsymbol{\theta}, \mathcal{J}(\boldsymbol{\theta})^{-1}\right)$.

\section{Variance Estimators}

We consider two estimators for the variance of the MLE $\hat{\boldsymbol{\theta}}$. The first is the modelbased estimator and is the asymptotic variance of $\hat{\boldsymbol{\theta}}$, or equivalently, the inverse of the expected Fisher information matrix. Note that if we take the variance of the Fisher scoring update (2.9), then upon convergence we have

$$
\begin{aligned}
\mathbf{V}_{\mathrm{MB}}(\hat{\boldsymbol{\theta}}) & =\operatorname{Cov}\left(\left[\mathbf{Z}^{\top} \mathbf{V}^{-1} \mathbf{Z}\right]^{-1} \mathbf{Z}^{\top} \mathbf{V}^{-1} \mathbf{Y}^{\star \star}\right) \\
& =\left[\mathbf{Z}^{\top} \mathbf{V}^{-1} \mathbf{Z}\right]^{-1} \mathbf{Z}^{\top} \mathbf{V}^{-1} \operatorname{Cov}\left(\mathbf{Y}^{\star \star}\right) \mathbf{V}^{-1} \mathbf{Z}\left[\mathbf{Z}^{\top} \mathbf{V}^{-1} \mathbf{Z}\right]^{-1} \\
& =\left[\mathbf{Z}^{\top} \mathbf{V}^{-1} \mathbf{Z}\right]^{-1} \\
& =\mathcal{J}(\boldsymbol{\theta})^{-1}
\end{aligned}
$$

where $\mathbf{V}^{-1}=\mathbf{V}^{\star \star}(\boldsymbol{\theta})^{-1}$ and $\mathbf{Z}=\mathbf{Z}(\boldsymbol{\theta})$ are evaluated at the true value $\boldsymbol{\theta}$. However, since the value of $\boldsymbol{\theta}$ is unknown, we can approximate (2.14) by replacing $\boldsymbol{\theta}$ with its 
MLE $\hat{\boldsymbol{\theta}}$ to get

$$
\hat{\mathbf{V}}_{\mathrm{MB}}(\hat{\boldsymbol{\theta}})=\mathcal{J}(\hat{\boldsymbol{\theta}})^{-1}
$$

We also consider the standard leave-one-out jackknife estimator. Let $\hat{\boldsymbol{\theta}}^{[-i]}$ be the estimate with the $i$-th data point removed and $\hat{\boldsymbol{\theta}}=\frac{1}{n} \sum_{i=1}^{n} \hat{\boldsymbol{\theta}}^{[-i]}$ their average, then the jackknife variance estimator is

$$
\hat{\mathbf{V}}_{\mathrm{JK}}(\hat{\boldsymbol{\theta}})=\frac{n-1}{n} \sum_{i=1}^{n}\left(\hat{\boldsymbol{\theta}}^{[-i]}-\hat{\boldsymbol{\theta}}\right)\left(\hat{\boldsymbol{\theta}}^{[-i]}-\hat{\boldsymbol{\theta}}\right)^{\top}
$$

Note that for ordinal data if the total number of observations is much greater than the number of unique covariates then the computational cost is reduced because there are many replicated covariates.

If $\hat{\mathbf{V}}(\hat{\boldsymbol{\theta}})$ is a variance estimator of $\hat{\boldsymbol{\theta}}$ (such as (2.15) or (2.16)), then the corresponding standard error estimate of the $k$-th component of $\hat{\boldsymbol{\theta}}$ is the square root of the $k$-th element of the diagonal of $\hat{\mathbf{V}}(\hat{\boldsymbol{\theta}}), \operatorname{se}\left(\hat{\theta}_{k}\right)=\sqrt{[\hat{\mathbf{V}}(\hat{\boldsymbol{\theta}})]_{k k}}$.

\section{Delta Method}

If $\hat{\mathbf{V}}(\hat{\boldsymbol{\theta}})$ is a consistent estimator of the covariance matrix $\operatorname{Cov}(\hat{\boldsymbol{\theta}})$ then $\hat{\boldsymbol{\theta}}$ has a $N(\boldsymbol{\theta}, \hat{\mathbf{V}}(\hat{\boldsymbol{\theta}}))$ asymptotic distribution. We use this property in conjunction with a Taylor-series expansion of a function of the estimator of $\boldsymbol{\theta}, h(\hat{\boldsymbol{\theta}})$, to approximate the distribution of $h(\hat{\boldsymbol{\theta}})$. This is commonly referred to as the delta method, see Bishop et al. (1975). 
First, we take a first-order Taylor-series expansion of $h(\hat{\boldsymbol{\theta}})$ about $\boldsymbol{\theta}$ :

$$
h(\hat{\boldsymbol{\theta}}) \approx h(\boldsymbol{\theta})+\frac{\partial h(\boldsymbol{\theta})}{\partial \boldsymbol{\theta}}(\hat{\boldsymbol{\theta}}-\boldsymbol{\theta}) .
$$

The right-hand side of (2.17) has an asymptotic normal distribution because the only

random component in the expression, $\hat{\boldsymbol{\theta}}$, is normally distributed. Taking the expectation and variance of $(2.17)$ gives $\mathrm{E}(h(\hat{\boldsymbol{\theta}})) \approx h(\boldsymbol{\theta})$ and $\operatorname{Cov}(h(\hat{\boldsymbol{\theta}})) \approx \frac{\partial h(\boldsymbol{\theta})}{\partial \boldsymbol{\theta}} \hat{\mathbf{V}}(\hat{\boldsymbol{\theta}}) \frac{\partial h(\boldsymbol{\theta})}{\partial \boldsymbol{\theta}^{\top}}$ for large samples. Again, since the true value $\boldsymbol{\theta}$ is unknown, we can replace $\boldsymbol{\theta}$ with its MLE, $\hat{\boldsymbol{\theta}}$; hence we approximate the variance by

$$
\hat{V}_{h}(\hat{\boldsymbol{\theta}})=\frac{\partial h(\hat{\boldsymbol{\theta}})}{\partial \boldsymbol{\theta}} \hat{\mathbf{V}}(\hat{\boldsymbol{\theta}}) \frac{\partial h(\hat{\boldsymbol{\theta}})}{\partial \boldsymbol{\theta}^{\top}}
$$

Thus the large-sample distribution of $h(\hat{\boldsymbol{\theta}})$ is approximately the normal distribution $h(\hat{\boldsymbol{\theta}}) \sim N\left(h(\boldsymbol{\theta}), \hat{V}_{h}(\hat{\boldsymbol{\theta}})\right)$.

\section{Confidence Intervals}

This asymptotic distribution is utilized in the Wald test statistic:

$$
\frac{h(\hat{\boldsymbol{\theta}})-h(\boldsymbol{\theta})}{\sqrt{\hat{V}_{h}(\hat{\boldsymbol{\theta}})}} \sim N(0,1)
$$

where $N(0,1)$ is a standard normal distribution. We can use (2.19) to construct a twosided confidence interval for $h(\boldsymbol{\theta})$ by considering the null hypothesis $H_{0}: h(\boldsymbol{\theta})=h\left(\boldsymbol{\theta}_{0}\right)$ versus the alternative hypothesis $H_{a}: h(\boldsymbol{\theta}) \neq h\left(\boldsymbol{\theta}_{0}\right)$. Then, a 100(1- $\left.\alpha\right) \%$ confidence interval is given by

$$
b_{d}=h(\hat{\boldsymbol{\theta}}) \pm z_{\alpha / 2} \sqrt{\hat{V}_{h}(\hat{\boldsymbol{\theta}})}
$$


where $z_{\alpha}$ is the $100(1-\alpha)$ percentile of a standard normal distribution.

\subsubsection{Model Selection Criteria}

Often statistical models require some form of selection criteria to compare one model to another and determine the optimal fit. A good selection criterion will balance the goodness-of-fit against the number of parameters required for the fit. There are several standard criteria for comparing models including Generalized cross-validataion (GCV)

$$
G C V(\boldsymbol{\theta})=\frac{\frac{1}{n(C-1)}(\mathbf{y}-\hat{\boldsymbol{\pi}})^{\boldsymbol{\top}}(\mathbf{y}-\hat{\boldsymbol{\pi}})}{\left(1-\frac{q}{n(C-1)}\right)^{2}},
$$

Akaike's information criteria (AIC)

$$
A I C(\boldsymbol{\theta})=2 q-2 l
$$

and Bayesian information criterion (BIC)

$$
B I C(\boldsymbol{\theta})=2 q \log n(C-1)-2 l
$$

where $l$ is the $\log$-likelihood and $q$ is the degrees of freedom or effective degrees of freedom for the model. With all these criteria a model with the lowest value is preferred. All of these approaches attempt to estimate the mean square error giving rise to the so-called bias-variance trade-off problem. The main difference between the three criteria above is how strongly they penalize the number of parameters. BIC is the most severe and so tends to pick simpler models than either the AIC or the GCV; GCV on average tends to fall in between the other two. 


\subsection{Monotone Smoothing Models for Ordinal Data}

We now consider modeling an ordinal response with a single covariate $x(r=1)$ by way of a monotone decreasing relationship. We use a model of the form (2.11),

$$
\operatorname{logit}[P(Y \leq j \mid x)]=\alpha_{j}+f(x), \quad j=1, \ldots, C-1,
$$

with the additional restriction that $f(x)$ is monotone decreasing. We will use the negation of (2.2) for the smooth function, $f(x)=-\int_{t_{1}}^{x} \exp (\boldsymbol{\Psi}(u) \boldsymbol{\beta}) \mathrm{du}$. Thus the model becomes

$$
\begin{aligned}
& \operatorname{logit}[P(Y \leq j)]=\eta_{j}(x) \\
& \eta_{j}(x)=\alpha_{j}-\int_{t_{1}}^{x} \exp (\Psi(u) \boldsymbol{\beta}) \mathrm{du}, \quad j=1, \ldots, C-1 .
\end{aligned}
$$

Note that subtracting the integral ensures that the probability of the first response category is monotone decreasing as a function of $x$. We denote the parameter vector for the model by $\boldsymbol{\theta}=\left(\alpha_{1}, \ldots, \alpha_{C-1}, \boldsymbol{\beta}^{\top}\right)^{\top}$.

In general, evaluating (2.21) will require quadrature to approximate the integral. Here however, we choose to use a piece-wise linear B-spline basis which eliminates the need for numerical integration and instead allows the integrals to be computed exactly. We have found that, within the dose-response context, such a basis appears to provide sufficient smoothness and flexibility to fit the model well. The definition for the B-spline basis of any degree was given in Section 2.2.1. Below, we present the 
linear B-spline basis used where

$$
\begin{gathered}
\Psi_{1}(u)=\left\{\begin{array}{l}
1-\frac{u-t_{1}}{t_{2}-t_{1}} \quad, \quad t_{1} \leq u<t_{2} \\
0 \quad, \text { otherwise }
\end{array}\right. \\
\Psi_{k}(u)= \begin{cases}\frac{u-t_{k-1}}{t_{k}-t_{k-1}}, & t_{k-1} \leq u<t_{k} \\
1-\frac{u-t_{k}}{t_{k+1}-t_{k}}, & t_{k} \leq u<t_{k+1}, \quad k=2, \ldots, K-1 \\
0 \quad, \quad \text { otherwise }\end{cases}
\end{gathered}
$$

and

$$
\Psi_{K}(u)=\left\{\begin{array}{ll}
\frac{u-t_{K-1}}{t_{K}-t_{K-1}} & , \quad t_{K-1} \leq u \leq t_{K} \\
0 & , \text { otherwise }
\end{array} .\right.
$$

Note that using a linear basis implies that for any $\boldsymbol{\beta}$ and $x \in \mathcal{D}$ the integral

$$
\int_{t_{1}}^{x} \exp (\boldsymbol{\Psi}(u) \boldsymbol{\beta}) \mathrm{du}=\sum_{\substack{k=1, \ldots, K-1 \\ t_{k} \leq x}} \int_{t_{k}}^{\min \left(t_{k+1}, x\right)} \exp (\boldsymbol{\Psi}(u) \boldsymbol{\beta}) \mathrm{du}
$$

can be divided into a number of subintegrals, split at the knots $t_{k}$. These integrals are either degenerate or the spline is linear over the relevant domain, and thus the 
components can be evaluated exactly since

$$
\int_{t_{k}}^{x} \exp (\boldsymbol{\Psi}(u) \boldsymbol{\beta}) \mathrm{du}= \begin{cases}\frac{t_{k+1}-t_{k}}{\beta_{k+1}-\beta_{k}} \exp \left(\beta_{k}\right)\left\{\exp \left[\frac{\left(x-t_{k}\right)\left(\beta_{k+1}-\beta_{k}\right)}{t_{k+1}-t_{k}}\right]-1\right\}, & \beta_{k} \neq \beta_{k+1} \\ \frac{1}{2}\left(x-t_{k}\right)\left[\exp \left(\beta_{k}\right)+\exp \left(\beta_{k+1}\right)\right], & \beta_{k}=\beta_{k+1}\end{cases}
$$

for $x \in\left[t_{k}, t_{k+1}\right), k=1, \ldots, K$. Thus we have a closed-form expression for the integral and consequently for the model (2.21).

Using piece-wise linear basis functions $\boldsymbol{\Psi}(x)$ implies that their first derivatives are discontinuous at the knot locations; thus the first derivatives of $\exp (\Psi(x) \boldsymbol{\beta})$ with respect to $x$ are also discontinuous at the same points. However, integrating $\exp (\boldsymbol{\Psi}(x) \boldsymbol{\beta})$ over $x$ smooths these discontinuities. This effectively adds an additional degree to the smoothness, thus (2.21) has one continuous derivative, namely $\exp (\boldsymbol{\Psi}(x) \boldsymbol{\beta})$. Of course, if we do require a smoother fit we could use B-splines of a higher degree but the approach would then require using a numerical quadrature.

In the remainder of this section we discuss estimation of (2.21) using an adaptive fixed knot approach. It can readily be generalized to (2.11) using various combinations of linear, smooth or monotone smooth functions for the additive predictors. In Section 2.3.2 we discuss a penalty-based approach.

The number and position of knots used in the basis must be given careful consideration. We investigate two different approaches to this matter, an adaptive fixed knot spline and a penalized spline, which we examine in Sections 2.3.1 and 2.3.2 respectively. Say we do not have a preconceived notion of the domain of variable, but do have a set of values $\mathcal{X}\left\{x_{1 s}, \ldots, x_{n s}\right\}$ from the variable. We can use the interval $\mathcal{D}=[\min \mathcal{X}, \max \mathcal{X}]$, which is the minimal connected set which span the range of the 
data, as the domain.

\subsubsection{Estimation via Adaptive Fixed Knots}

\section{Maximum Likelihood Estimation with Fixed Knots}

For a given set of knots, we can estimate $\boldsymbol{\theta}$ via the maximum likelihood procedure described in Section 2.2.3. To do so we only require an initial estimate $\boldsymbol{\theta}_{[0]}$, and an expression for $\frac{\partial \boldsymbol{\eta}_{i}}{\partial \boldsymbol{\theta}}$.

In (2.13) we use the gradient

$$
\frac{\partial \boldsymbol{\eta}_{i}}{\partial \boldsymbol{\theta}}=\left[\begin{array}{l|c} 
& -\boldsymbol{\Omega}\left(x_{i}, \boldsymbol{\beta}\right) \\
\mathbf{I}_{C-1} & \vdots \\
& \boldsymbol{\Omega}\left(x_{i}, \boldsymbol{\beta}\right)
\end{array}\right]
$$

where the row vector $\boldsymbol{\Omega}(x, \boldsymbol{\beta})=\left(\Omega_{1}(x, \boldsymbol{\beta}), \ldots, \Omega_{K}(x, \boldsymbol{\beta})\right)$ is the gradient of $f$ with respect to $\boldsymbol{\beta}$ with components $\Omega_{k}(x, \boldsymbol{\beta})=\int_{t_{1}}^{x} \exp (\boldsymbol{\Psi}(u) \boldsymbol{\beta}) \Psi_{k}(u)$ du. Similar to the integral in (2.22), as well as the $\boldsymbol{\eta}_{i}$ themselves, the $\boldsymbol{\Omega}\left(x_{i}, \boldsymbol{\beta}\right)$ can also be expressed in closed form, differentiating directly from (2.22).

To use the Fisher scoring method described in Section 2.2.3 (or any iterative optimization procedure) we require an initial estimate $\boldsymbol{\theta}_{[0]}$. We obtain this initial estimate by fitting a univariate GLM with a logit link,

$$
\operatorname{logit}[P(Y \leq j)]=\alpha_{j}+w \nu
$$

where $w=t_{1}-x$ to each of the $j=1, \ldots, C-1$ cumulative probabilities arising from the data. This differs from CUR in that the covariance structure within each $\mathbf{Y}_{i}^{\star}$ is ignored. That is, for this initialization procedure, $\operatorname{Cov}\left(\mathbf{Y}^{\star \star}\right)$ is assumed to be 
$\operatorname{diag}\left\{\boldsymbol{\pi}^{\star}(1-\boldsymbol{\pi})^{\star}\right\}$ instead of having the block diagonal of $\mathbf{V}^{\star \star}(\boldsymbol{\theta})$. Alternatively, we could first fit a CUR model and obtain our initial estimates in the same manner. We use the estimates obtained for the intercepts from the fit directly, $\boldsymbol{\alpha}_{[0]}=\hat{\boldsymbol{\alpha}}$, and then estimate the spline term as a constant function by setting each $\beta_{[0] k}=\log (\hat{\nu})$. Combined these give our initial estimate $\boldsymbol{\theta}_{[0]}=\left(\boldsymbol{\alpha}_{[0]}^{\top}, \boldsymbol{\beta}_{[0]}^{\top}\right)^{\top}$. This method will not result in an initial estimate if $\hat{\nu}<0$, however in such cases the data is likely not monotone decreasing with the $x_{i}$ 's. Also, the resulting starting values are rough estimates, however the multinomial likelihood is convex (see Pratt, 1981) and so convergence is guaranteed with gradient ascent algorithms. We refer to this method, where we estimate the model with a predetermined set of fixed knots, as FXMS.

\section{Adaptive Procedure}

So far we have assumed that the knot sequence $\mathbf{t}$ is known, however selecting the number and location of these knots is a non-trivial problem.

Intuitively, a natural approach to the problem would be to optimize the knots concurrently with $\boldsymbol{\theta}$, and such an approach is referred to as a free knot spline. However, finding the optimal knot locations via direct optimization is not a simple task, presenting a host of problems. One of those problems is knots collasing: if $t_{k}=t_{k+1}$ for some $k$ then the spline has one less continuous derivative at $t_{k}$. Unless specifically desired, this is a behaviour we would like to avoid. Optimization of free knot splines also has a tendency to have many local optima, making convergence to a global maximum challenging.

Another approach is discretization of the domain of the spline into a collection of points, $\mathcal{T}$, then fit the model using a subset of size $k-2$ from $\mathcal{T}$ as the interior knots. Once all the models have been fit, one can choose the model and corresponding knots 
which resulted in the highest likelihood. However, if the size of $\mathcal{T}$ or $k$ is moderately large then the number of permutations necessary can quickly become untenable. As and alternative, one can consider instead of all subsets, to take a random subset selection of knots from $\mathcal{T}$, see Kooperberg et al. (1997) for details. However, even this approach suffers from combinatorial explosion for large $\mathcal{T}$ and $k$.

To choose the number of knots as well as their position one can consider knot insertion/deletion routines similar to forward and backward parameter selection in regression modeling. The fits can them be compared via one of the selection criteria presented in Section 2.2.5

Here, with a monotonicity constraint, the complexity of the number and position of the knots is reduced. If relatively few knots are needed then a discretization approach is feasible and is the approach we recommend here. We first focus on choosing near optimal knots when we have a fixed number of interior knots. We select a potential set of knots, possibly evenly spaced over the range of $\mathbf{x}$. We then select each possible subset of the specified number of interior knots, combine it with the boundary knots, fit the model and record the values of the selection criteria. Next we compare the criteria and pick the set of knots which resulted in the lowest value; these knots are the near optimal knot locations. It is possible to repeat the process refining the potential knot set if desired. We can also follow the same process for different numbers of interior knots. Generally we would like to keep the number of interior knots small. We can again compare the near optimal selection for each number of interior knots via a selection criteria. Some examples of such criteria are given in Section 2.2.5. We refer to models estimated via the adaptive fixed knot approach as AMS.

We also need to consider the maximum number of knots given the degrees of freedom available for the data. The structure of the integral in (2.21) ensures that 
$f\left(t_{1}\right)=0$, and if the boundary knots are not selected apriori but rather determined by the data, then this induces a constraint on the parameter estimates and thus a degree of freedom. If we let $n_{x}$ be the number of distinct values in $\mathbf{x}$ then the number of columns in the basis, $m+K-2$, must be no more than $n_{x}-1$.

\subsubsection{Estimation via Penalized Splines}

Since finding optimal knots is a hard problem we can instead consider penalized smoothing, where we estimate the model (2.21) via the penalized likelihood method Eilers and Marx (1996). The number and location of knots when using a penalty is far less crucial than in the fixed knot case as a large number of knots are used and the degrees of freedom regularized via the penalty. In the penalized approach one uses a large number of knots, typically either evenly spaced over the range of $\mathbf{x}$ or at the quantiles. Following Ruppert (2002), we use $\min \left(40, \frac{n_{x}}{4}\right)$ knots located at the quantiles of $\mathbf{x}$.

\section{Penalized Maximum Likelihood}

Estimation is similar to the approach presented in 2.2.3, but instead of maximizing the log-likelihood, we maximize the penalized log-likelihood,

$$
\begin{aligned}
l_{\mathrm{PEN}}(\boldsymbol{\theta}) & =l(\boldsymbol{\theta})-\frac{1}{2} \lambda \boldsymbol{\theta}^{\boldsymbol{\top}} \mathbf{P} \boldsymbol{\theta} \\
& =\left[\sum_{i=1}^{n} \mathbf{y}_{i}^{\star \top} \boldsymbol{\phi}_{i}(\boldsymbol{\theta})-b\left(\boldsymbol{\phi}_{i}(\boldsymbol{\theta})\right)\right]-\frac{1}{2} \lambda \boldsymbol{\theta}^{\boldsymbol{\top}} \mathbf{P} \boldsymbol{\theta},
\end{aligned}
$$


where $l(\boldsymbol{\theta})$ is the unpenalized log-likelihood in (2.4),

$$
\mathbf{D}_{\beta}=\left[\begin{array}{rrrrr}
-1 & 1 & & & 0 \\
& -1 & 1 & & \\
& & \ddots & \ddots & \\
0 & & & -1 & 1
\end{array}\right]
$$

is a $K-1 \times K$ matrix of first order differences, $\mathbf{P}=\operatorname{blkdiag}\left\{\mathbf{0}_{C-1}, \mathbf{D}_{\beta}^{\top} \mathbf{D}_{\beta}\right\}$ is the penalty matrix and $\lambda$ is the smoothing parameter. This penalty is a finite difference approximation and we penalize the first order differences because (2.21) with a linear basis has a single continuous derivative. With a different basis one may use a different penalty were appropriate, such as the second order differences which approximates a penalty on the second derivative of the function.

The gradient and Hessian are given by

$$
\frac{\partial l_{\mathrm{PEN}}(\boldsymbol{\theta})}{\partial \boldsymbol{\theta}^{\top}}=\frac{\partial l(\boldsymbol{\theta})}{\partial \boldsymbol{\theta}^{\top}}-\lambda \mathbf{P} \boldsymbol{\theta}
$$

and

$$
\frac{\partial^{2}}{\partial \boldsymbol{\theta} \partial \boldsymbol{\theta}^{\top}} l_{\mathrm{PEN}}(\boldsymbol{\theta})=\frac{\partial^{2}}{\partial \boldsymbol{\theta} \partial \boldsymbol{\theta}^{\top}} l(\boldsymbol{\theta})-\lambda \mathbf{P}
$$

respectively.

Recalling that $\frac{\partial l(\boldsymbol{\theta})}{\partial \boldsymbol{\theta}^{\top}}=\mathbf{Z}(\boldsymbol{\theta})^{\top} \mathbf{V}^{\star \star}(\boldsymbol{\theta})^{-1}\left(\mathbf{y}^{\star \star}-\boldsymbol{\pi}^{\star \star}(\boldsymbol{\theta})\right)$ is the gradient of the nonpenalized $\log$-likelihood and that $\mathcal{J}(\boldsymbol{\theta})=\mathrm{E}\left(-\frac{\partial^{2} l(\boldsymbol{\theta})}{\partial \boldsymbol{\theta} \partial \boldsymbol{\theta}^{\top}}\right)=\mathbf{Z}(\boldsymbol{\theta})^{\top} \mathbf{V}^{\star \star}(\boldsymbol{\theta})^{-1} \mathbf{Z}(\boldsymbol{\theta})$ is the expected Fisher information matrix, we also have

$$
\mathcal{J}_{\mathrm{PEN}}(\boldsymbol{\theta})=\mathrm{E}\left(-\frac{\partial^{2} l_{\mathrm{PEN}}(\boldsymbol{\theta})}{\partial \boldsymbol{\theta} \partial \boldsymbol{\theta}^{\top}}\right)=\mathbf{Z}(\boldsymbol{\theta})^{\top} \mathbf{V}^{\star \star}(\boldsymbol{\theta})^{-1} \mathbf{Z}(\boldsymbol{\theta})+\lambda \mathbf{P} .
$$


Consequently, the Fisher scoring update for the penalized log-likelihood is

$$
\begin{aligned}
\boldsymbol{\theta}_{[t+1]} & =\boldsymbol{\theta}_{[t]}+\mathcal{J}_{\mathrm{PEN}}\left(\boldsymbol{\theta}_{[t]}\right)^{-1} \frac{\partial l_{\mathrm{PEN}}\left(\boldsymbol{\theta}_{[t]}\right)}{\partial \boldsymbol{\theta}^{\top}} \\
& =\boldsymbol{\theta}_{[t]}+\left[\mathbf{Z}\left(\boldsymbol{\theta}_{[t]}\right)^{\top} \mathbf{V}^{\star \star}\left(\boldsymbol{\theta}_{[t]}\right)^{-1} \mathbf{Z}\left(\boldsymbol{\theta}_{[t]}\right)+\lambda \mathbf{P}\right]^{-1} \mathbf{Z}\left(\boldsymbol{\theta}_{[t]}\right)^{\top} \mathbf{V}^{\star \star}\left(\boldsymbol{\theta}_{[t]}\right)^{-1}\left[\mathbf{y}^{\star \star}-\boldsymbol{\pi}^{\star \star}\left(\boldsymbol{\theta}_{[t]}\right)\right] \\
& =\left[\mathbf{Z}\left(\boldsymbol{\theta}_{[t]}\right)^{\top} \mathbf{V}^{\star \star}\left(\boldsymbol{\theta}_{[t]}\right)^{-1} \mathbf{Z}\left(\boldsymbol{\theta}_{[t]}\right)+\lambda \mathbf{P}\right]^{-1} \mathbf{Z}\left(\boldsymbol{\theta}_{[t]}\right)^{\top} \mathbf{V}^{\star \star}\left(\boldsymbol{\theta}_{[t]}\right)^{-1} \widetilde{\mathbf{y}}^{\star \star}\left(\boldsymbol{\theta}_{[t]}\right) .
\end{aligned}
$$

We will refer to models estimated in this manner as a penalized monotone spline (PMS).

\section{Controlling the Amount of Smoothing}

The smoothing parameter $\lambda$ must be estimated. A large value for $\lambda$ will reduce the model to the cumulative logit model, whereas a small value for $\lambda$ will approach an interpolant of the data. The minimum effective degrees of freedom for the model is $C$ while the maximum is $n_{x}+C-1$. We choose $\lambda$ based on some criterion such as those discussed in Section 2.2.5. First we fit the model using several values of $\lambda$ ranging from very small to very large and then refine the search around the lowest value obtained.

Unlike all the previous models discussed, the PMS does not have a fixed degrees of freedom. We can approximate the degrees of freedom by the trace of the influence matrix. We can obtain the influence matrix for the linear approximation to our model, namely $\mathbf{H}=\hat{\mathbf{Z}}\left[\hat{\mathbf{Z}}^{\top} \hat{\mathbf{V}}^{-1} \hat{\mathbf{Z}}+\hat{\lambda} \mathbf{P}\right]^{-1} \hat{\mathbf{Z}}^{\top} \hat{\mathbf{V}}^{-1}$ where $\hat{\mathbf{Z}}$ and $\hat{\mathbf{V}}^{-1}$ are evaluated at the converged estimate $\hat{\boldsymbol{\theta}}$. Then we can approximate the effective degrees of freedom by $\operatorname{tr}\{\mathbf{H}\}=\operatorname{tr}\left\{\hat{\mathbf{Z}}\left[\hat{\mathbf{Z}}^{\top} \hat{\mathbf{V}}^{-1} \hat{\mathbf{Z}}+\lambda \mathbf{P}\right]^{-1} \hat{\mathbf{Z}}^{\top} \hat{\mathbf{V}}^{-1}\right\}=\operatorname{tr}\left\{\left[\hat{\mathbf{Z}}^{\top} \hat{\mathbf{V}}^{-1} \hat{\mathbf{Z}}+\lambda \mathbf{P}\right]^{-1} \hat{\mathbf{Z}}^{\top} \hat{\mathbf{V}}^{-1} \hat{\mathbf{Z}}\right\}$. 


\section{Variance Estimator}

The model-based variance of $\hat{\boldsymbol{\theta}}$ for the penalized model is based on $\mathcal{J}_{\mathrm{PEN}}(\boldsymbol{\theta})$. The estimator is

$$
\begin{aligned}
\mathbf{V}_{\mathrm{PEN}}(\hat{\boldsymbol{\theta}}) & =\operatorname{Cov}\left(\left[\mathbf{Z}^{\top} \mathbf{V}^{-1} \mathbf{Z}+\lambda \mathbf{P}\right]^{-1} \mathbf{Z}^{\top} \mathbf{V}^{-1} \mathbf{Y}^{\star \star}\right) \\
& =\left[\mathbf{Z}^{\top} \mathbf{V}^{-1} \mathbf{Z}+\lambda \mathbf{P}\right]^{-1} \mathbf{Z}^{\top} \mathbf{V}^{-1} \operatorname{Cov}\left(\mathbf{Y}^{\star \star}\right) \mathbf{V}^{-1} \mathbf{Z}\left[\mathbf{Z}^{\top} \mathbf{V}^{-1} \mathbf{Z}+\lambda \mathbf{P}\right]^{-1} \\
& =\left[\mathbf{Z}^{\top} \mathbf{V}^{-1} \mathbf{Z}+\lambda \mathbf{P}\right]^{-1} \mathcal{J}(\boldsymbol{\theta})\left[\mathbf{Z}^{\top} \mathbf{V}^{-1} \mathbf{Z}+\lambda \mathbf{P}\right]^{-1} .
\end{aligned}
$$

and is approximated by

$$
\hat{\mathbf{V}}_{\mathrm{PEN}}(\hat{\boldsymbol{\theta}})=\left[\hat{\mathbf{Z}}^{\top} \hat{\mathbf{V}}^{-1} \hat{\mathbf{Z}}+\lambda \mathbf{P}\right]^{-1} \mathcal{J}(\hat{\boldsymbol{\theta}})\left[\hat{\mathbf{Z}}^{\top} \hat{\mathbf{V}}^{-1} \hat{\mathbf{Z}}+\lambda \mathbf{P}\right]^{-1}
$$

\subsection{Data Analysis}

We fit a number of models to data which appear in USEPA (2010) and concern the incidences of selected histopathological lesions in rats exposed to dietary 1,1,2,2tetrachlorethane at 14 weeks. Lesions that were observed were categorized as one of Cytoplasmic vacuolization, Hypertrophy, Necrosis, Pigmentation or Mitotic alteration. These data are reproduced in Table 2.1.

Summaries of these fits are given in Table 2.2 and in Figure 2.1. From both the table and figures it is clear that the CUR model performs poorly while the AMS and PMS models, both of which employ monotone splines, fit well. In figure Figure 2.2 we also fit the data using non-monotone splines denoted by FXS and PS for the fixed knot and penalized cases respectively. It is clear that imposing monotoniciy is necessary. If 


\begin{tabular}{lcccccc}
\hline \multirow{2}{*}{$\begin{array}{c}\text { Dose } \\
(\mathrm{mg} / \mathrm{kg}-\mathrm{d})\end{array}$} & $\begin{array}{c}\text { Cytoplasmic } \\
\text { vacuolization }\end{array}$ & $\begin{array}{c}\text { Hyper- } \\
\text { trophy }\end{array}$ & Necrosis & $\begin{array}{c}\text { Pigment- } \\
\text { ation }\end{array}$ & $\begin{array}{c}\text { Mitotic } \\
\text { alteration }\end{array}$ & Total \\
\hline 20 & 7 & 0 & 0 & 0 & 0 & 7 \\
40 & 19 & 0 & 0 & 0 & 0 & 19 \\
80 & 20 & 5 & 1 & 0 & 0 & 26 \\
170 & 12 & 19 & 15 & 17 & 11 & 74 \\
320 & 0 & 20 & 20 & 20 & 16 & 76 \\
\hline
\end{tabular}

Table 2.1: Incidences of selected histopathological lesions in rats exposed to dietary 1,1,2,2-tetrachlorethane for 14 weeks (USEPA, 2010).

not then undesirable non-monotonicity will almost always occur as this is the natural tendency of splines as they are smoothly connected piecewise polynomials.

\begin{tabular}{|c|c|c|c|c|c|c|c|c|}
\hline \multirow{2}{*}{ Model } & \multicolumn{2}{|c|}{ Basis } & \multicolumn{3}{|c|}{ df } & \multicolumn{3}{|c|}{ Criteria } \\
\hline & Degree & Intercept & $\alpha$ & $f$ & Total & GCV & AIC & BIC \\
\hline CUR & - & - & 4 & - & 5 & 0.704 & 552.149 & 577.879 \\
\hline AS & 2 & No & 4 & 3 & 7 & 0.671 & 507.263 & 543.286 \\
\hline AMS & 1 & Yes & 4 & 3 & 7 & 0.671 & 507.278 & 543.301 \\
\hline PS & 3 & No & 4 & 2.612 & 6.612 & 0.668 & 506.859 & 540.885 \\
\hline PMS & 1 & Yes & 4 & 2.521 & 6.521 & 0.668 & 506.692 & 540.252 \\
\hline
\end{tabular}

Table 2.2: Summary of model properties and selection criteria for fits to the 1,1,2,2-tetrachlorethane data. 


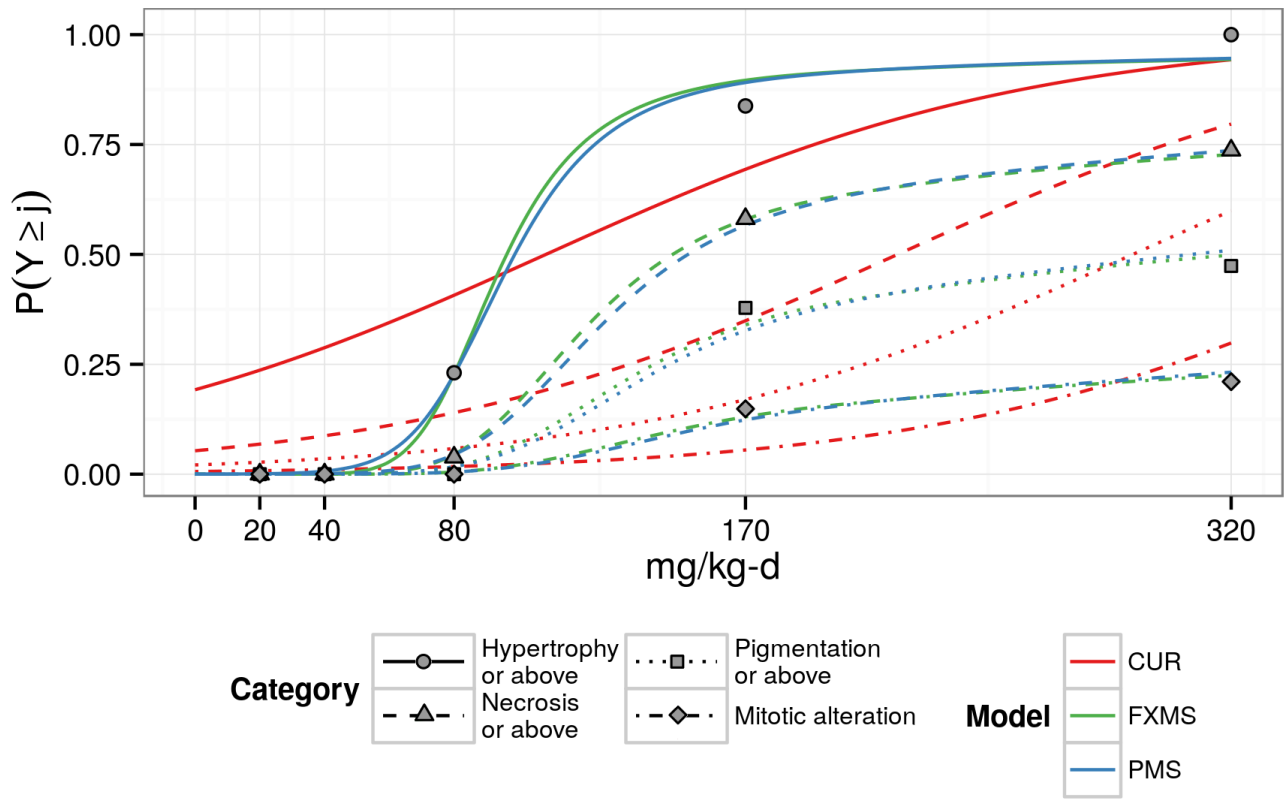

Figure 2.1: Cumulative probability estimates of CUR, AMS and PMS fits to the 1,1,2,2-tetrachlorethane data. The model fits are represented by lines while the data themselves are displayed as points. 


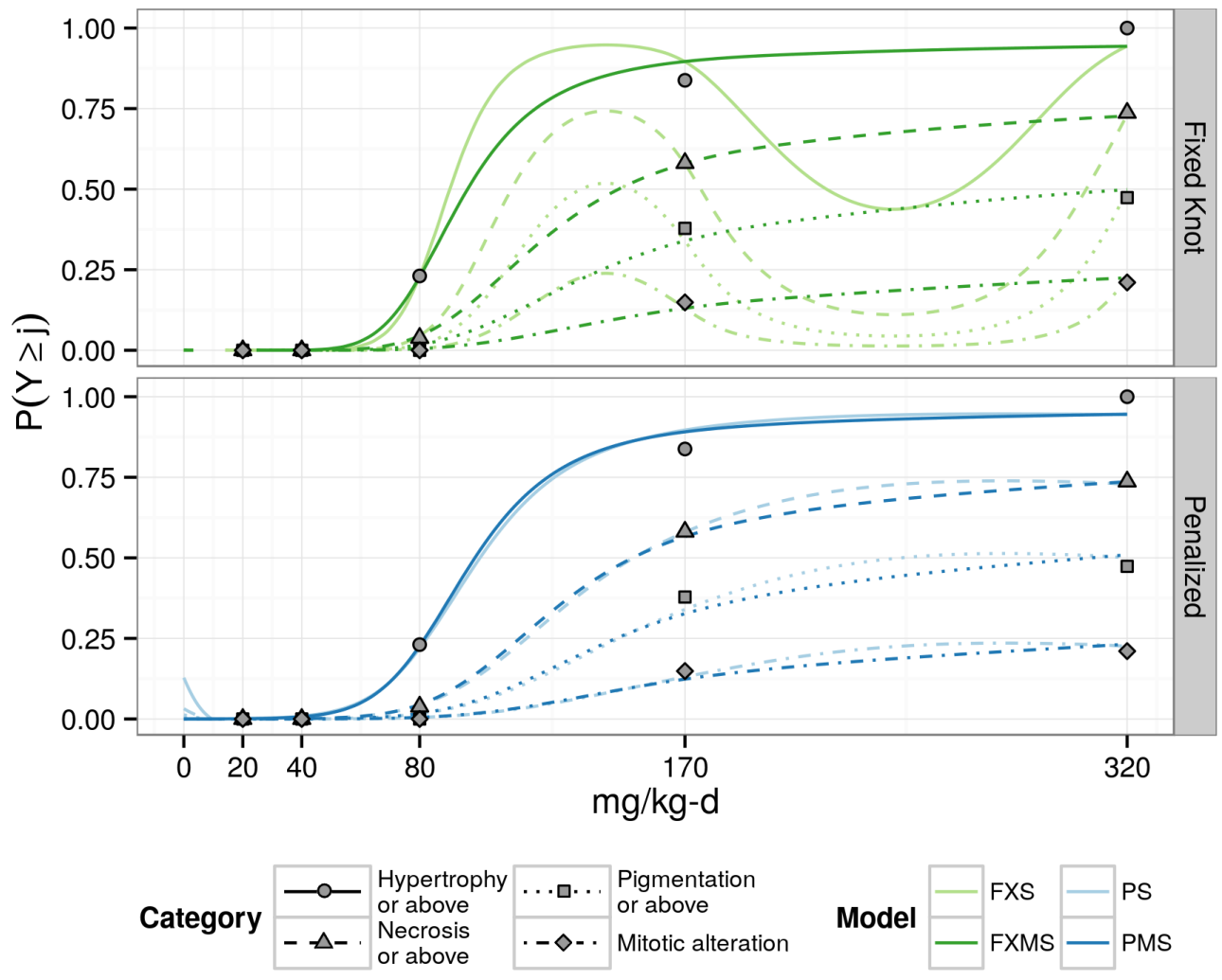

Figure 2.2: Cumulative probability estimates for AS, AMS, PS and PMS fits to the 1,1,2,2-tetrachlorethane data. The model fits are represented by lines while the data themselves are displayed as points. 


\subsection{Simulation}

In this section, we conduct a number of simulation studies that aim to assess the performance of CUR, FXMS, AMS and PMS models in terms of a number of different attributes. Initially, in Section 2.5.1, we investigate the model performance of the CUR and PMS models in two contrasting scenarios; in the first the data arises from a CUR model while in the second the CUR model is misspecified and emulates a dosefinding study. Secondly, in Section 2.5.2, two separate simulations are performed to assess the properties of the estimators of the log odds of the cumulative probability,

$\eta_{i j}$, for the adaptive fixed knot and penalized approaches of estimating (2.21). All simulations were performed using the R software language (R Core Team, 2015) and an $\mathrm{R}$ package which implements the models and results in this thesis will be made available.

\subsubsection{Emulating a Dose-finding Study}

In this section, we begin by presenting the results of a first simulation that compares the performance of the CUR and PMS models when the data are assumed to arise form a CUR model. This investigation will allow for an assessment of the behaviour of the PMS model when it is not the underlying mechanism for data generation.

We describe the cumulative probabilities for all categories but the last category by:

$$
\dot{\eta}_{j}(x)=\operatorname{logit}[P(Y \leq j \mid x)]=j-0.5-\frac{x}{200}, j=1,2,3
$$


or equivalently

$$
\dot{\pi}_{j}(x)=P(Y=j \mid x)=\left\{\begin{array}{cc}
\operatorname{logit}^{-1}\left(0.5-\frac{x}{200}\right) & , j=1 \\
\operatorname{logit}^{-1}\left(1.5-\frac{x}{200}\right)-\operatorname{logit}^{-1}\left(0.5-\frac{x}{200}\right) & , j=2 \\
\operatorname{logit}^{-1}\left(2.5-\frac{x}{200}\right)-\operatorname{logit}^{-1}\left(1.5-\frac{x}{200}\right) & , j=3 \\
1-\operatorname{logit}^{-1}\left(2.5+\frac{x}{200}\right) & , j=4
\end{array}\right.
$$

where $C=4$ is the total number of categories. This is a CUR model with parameter $\dot{\boldsymbol{\theta}}=\left(-0.5,0.5,1.5,-\frac{1}{200}\right)^{\top}$. Note that the expression for $\dot{\eta}_{j}(x)$ in $(2.26)$ is linear in the dose covariate $x$.

For the simulation, we consider a setup with $n=350$; specifically where there are seven dosages, $\{0,10,25,50,100,150,200\}$, and 50 observations at each dose and thus define $\mathbf{x}=(0,10,25,50,100,150,200)^{\top} \otimes \mathbf{1}_{50}$ to be a vector of the covariates. The true value of $\dot{\eta}_{j}(x)(j=1,2,3)$ has been computed using (2.26), and plotted over the domain $[0,200]$ in the diagram in the left panel of Figure 2.3. The points represent the values at the seven dose levels chosen for the simulation.

To create a simulated data set from the CUR model we generate a multinomial variate $\mathbf{y}_{i}$ from the probabilities in (2.27) for each of the dose levels $x_{i}, i=1, \ldots, n$. We generate 1,000 such data sets and fit both the CUR and PMS models to each. For both the CUR and PMS models, we evaluate the AIC, for each of the 1,000 simulated data sets, and determine their means. These are presented on the left side of Table 2.3. The mean AICs under the two models are nearly identical, demonstrating the robustness of the PMS model in this situation. 


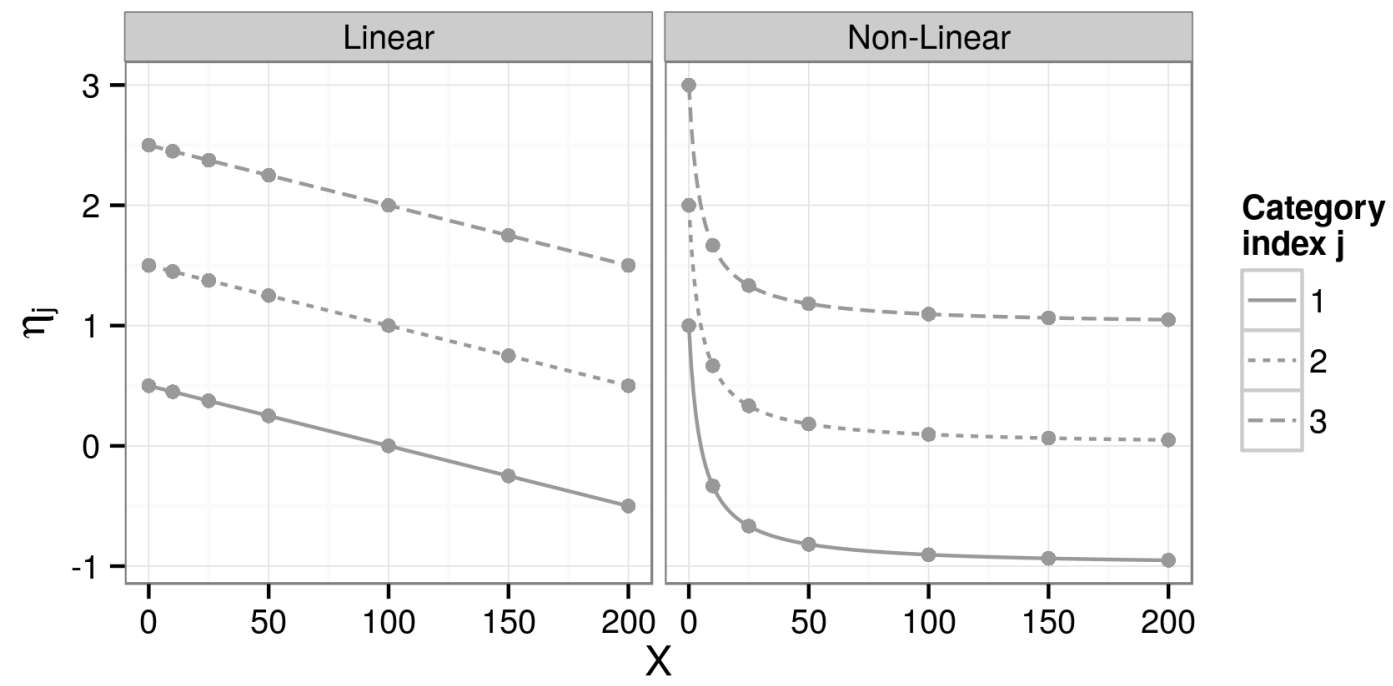

Figure 2.3: Plots of $\dot{\eta}_{j}(x),(j=1,2,3)$, given in (2.26) and (2.28) in the left panel and right panels, respectively.

\begin{tabular}{ccccccc}
\hline & \multicolumn{2}{c}{ Linear } & & \multicolumn{2}{c}{ Non-Linear } \\
\cline { 2 - 3 } \cline { 5 - 6 } & CUR & PMS & & CUR & PMS \\
\hline AIC & 956.3 & 955.7 & & 930.6 & 909.4 \\
\hline
\end{tabular}

Table 2.3: Mean AIC of CUR and PMS fits over 1,000 simulated replicates generated from the probabilities (2.27) and (2.29) on the left and right respectively. 
For each of the simulated data sets, we also determined estimates of $\boldsymbol{\theta}$ under both the CUR and PMS models, and used these to calculate an estimate, $\hat{\boldsymbol{\eta}}^{[m]}(x)$, $m=1, \ldots, N$, of $\dot{\eta}_{j}(x)$ over the domain $[0,200]$ for each model. Then, for each model, we found the average estimate, $\overline{\boldsymbol{\eta}}(x)=\frac{1}{N} \sum_{m=1}^{N} \hat{\boldsymbol{\eta}}^{[m]}(x)$, over the simulated data sets, and since $\dot{\eta}_{j}(x)$ is known, used those averages to determine an estimate of the bias in the estimator of $\dot{\eta}_{j}(x)$. Specifically, we obtained these estimates by approximating the expected value that appears in Section 2.2.4, $\mathrm{E}\left(\hat{\eta}_{j}(x)\right)$, by $\overline{\boldsymbol{\eta}}(x)$. These are plotted in the left panel of Figure 2.4; we note that the PMS model is very comparable to the CUR model and both appear to be unbiased.



Figure 2.4: Estimated bias of $\hat{\eta}_{j}(x),(j=1,2,3)$, for the CUR and PMS models over 1,000 simulated replicates generated from (2.27) and (2.29) in the left and right panels respectively. 




Figure 2.5: Estimated coverage of $\hat{\eta}_{j}(x),(j=1,2,3)$, for the CUR and PMS models over 1,000 simulated replicates generated from (2.27) and (2.29) in the left and right panels respectively.

Finally, for both models, not only is an estimate of $\dot{\eta}_{j}(x)$ available for each simulated data set, but its estimated asymptotic standard error has been determined as well. This standard error is the square root of the variance estimate which is derived using the delta method described in Section 2.2.4; we apply the method by taking $h=\eta_{j}$ and $\hat{\mathbf{V}}(\hat{\boldsymbol{\theta}})$ from the appropriate model-based estimates, $\hat{\mathbf{V}}_{\mathrm{MB}}(\hat{\boldsymbol{\theta}})$ in (2.15) for CUR, and $\hat{\mathbf{V}}_{\text {PEN }}(\hat{\boldsymbol{\theta}})$ in (2.25) for PMS. Thus, for each of the 1,000 simulated data sets, we were able to compute, under both models, a 95\% standard normal confidence interval for $\eta_{j}(x)$ over the domain $[0,200]$. Since $\dot{\eta}_{j}(x)$ is known, we use these confidence intervals to determine coverage rates for the estimators of $\dot{\eta}_{j}(x)$ under both the PMS and CUR models. These are plotted in the left panel of Figure 2.5. Here we observe that the CUR model has the expected coverage and that the PMS model has slightly poorer coverage than CUR for some values of $x$.

We also conduct a second simulation where data are no longer assumed to arise 
from a CUR model. Specifically, the functions

$$
\dot{\eta}_{j}(x)=\operatorname{logit}[P(Y \leq j \mid x)]=\frac{10}{x+5}-1+j, j=1,2,3,
$$

or equivalently

$$
\dot{\pi}_{j}(x)=P(Y=j \mid x)=\left\{\begin{array}{cc}
\operatorname{logit}^{-1}\left(-0.5+\frac{10}{x+5}\right) & , j=1 \\
\operatorname{logit}^{-1}\left(0.5+\frac{10}{x+5}\right)-\operatorname{logit}^{-1}\left(-0.5+\frac{10}{x+5}\right) & , j=2 \\
\operatorname{logit}^{-1}\left(1.5+\frac{10}{x+5}\right)-\operatorname{logit}^{-1}\left(0.5+\frac{10}{x+5}\right) & , j=3 \\
1-\operatorname{logit}^{-1}\left(1.5+\frac{10}{x+5}\right) & , j=4
\end{array}\right.
$$

describe the true underlying probabilities for this second scenario.

The $\dot{\eta}_{j}(x)$ are plotted in the diagram in the right panel of Figure 2.3; for all $(j=1,2,3)$, these functions drop sharply between $x=0$ and $x=50$, and then plateaus as $x$ increases further. We chose this shape to approximately emulate the data that resulted the study with ClinicalTrials.gov identifier NCT00413660. This was a Phase II double-blind study to compare six dosages of tofacitinib against a placebo for the efficacy of treating rhematoid arthritis and is discussed in Kremer et al. (2012). The response variable for this study was the American College of Rheumatology (ACR) improvement criteria level which was achieved by the study patients. The first response category was that of non-response, corresponding to those patients who observed less than a $20 \%$ improvement; the other categories are defined by achieving at least a $20 \%$ improvement (ACR20), a 50\% improvement (ACR50) and a $70 \%$ 
improvement (ACR70).

We follow a procedure analogous to the previous simulation for generating data and fitting the models, replacing the probabilities in (2.27) by those in (2.29). The dosages and the number of observations we use in both simulations, similarly to the shape, were chosen to be comparable to those in the Phase II double blind study described above.

The construction of the models is such that we expect the PMS to be adept at handling arbitrary smooth functions and for the CUR to struggle in the same situation; the results of this simulation lend support to that expectation. From the right side of Table 2.3, we note that the AIC for the PMS is noticeably lower, indicating a better fit. This is also apparent from the mean bias plot in the right panel of Figure 2.4; the PMS has a much smaller mean bias than CUR across the whole domain of $x$. Finally, note from the right panel of Figure 2.5 that PMS provides coverage which is close to the nominal value of $95 \%$ over the majority of the domain of $x$ whereas the CUR model struggles to attain the nominal coverage over most of the domain.

In summary, from the first simulation we observed that the CUR model fit and performed well when it is correctly specified, however it lacks the flexibility to fit the second simulation. On the other hand, the PMS model fits well, exhibits little bias, and good coverage, in both cases.

\subsubsection{Simulation Investigating Estimator Properties}

In this section we perform two separate sets of simulations which are intended to investigate the performance with regards to sample size of each of the FXMS and PMS models, when the model is correctly specified. For both simulations, we consider a scenario with data that has $C=3$ categories and a single covariate $x$ with domain 
$[0,1]$.

The first simulation investigates the FXMS model. We let the true underlying model be described by (2.21) with parameter vectors $\dot{\boldsymbol{\alpha}}=(0,2.0)^{\top}$ and $\dot{\boldsymbol{\beta}}=$ $\{\log 5, \log 0.5, \log 5\}^{\top}$ and knot sequence $\dot{\mathbf{t}}=\{0,0.5,1\}$, which contains a single interior knot located at the midpoint of the range. The $\dot{\eta}_{j}(x)$ corresponding to this setup are presented in Figure 2.6.

We examine the performance of the FXMS model under a $3 \times 3$ factorial of designs; specifically where the number of doses is one of $\{5,20,100\}$ and the number of replications per dose is one of $\{20,50,100\}$. For all designs we define a vector $\mathbf{x}$ with dose locations that are equally spaced over the domain $[0,1]$ and repeated the specified number of times. For each of these nine designs we generate 1,000 data sets from (2.21) using parameters $\dot{\boldsymbol{\alpha}}$ and $\dot{\boldsymbol{\beta}}$, covariates $\mathbf{x}$ and knots t. For each data set, we fit an FXMS model using the same set of knots, $\dot{\mathbf{t}}$, and compute the resulting model parameter estimates, as well as the model-based and jackknife standard error estimates.

For each simulated data set associated with a given design distinguished by the number of dose levels and replications per dose, we calculated, for any $x$ within the domain $[0,1]$, the estimates $\hat{\eta}_{j}(x)$ of $\eta_{j}(x)$. We used these results to study various characteristics of $\hat{\eta}_{j}(x)$ : the bias; the variance, which is investigated through $\hat{\mathbf{V}}_{\mathrm{MB}}\left(\hat{\eta}_{j}(x)\right)$ and $\hat{\mathbf{V}}_{\mathrm{JK}}\left(\hat{\eta}_{j}(x)\right)$ from Equations (2.15) and (2.16) respectively; and the coverage properties of the $95 \%$ confidence intervals in (2.20) which are based on the standard normal distribution.

For each design considered here, the nine panels in Figure 2.7 present estimates of the bias in $\hat{\eta}_{j}(x)$. From this figure, there appears to be some bias for smaller sample sizes but it becomes negligible for large sample sizes. 
In Figure 2.8 we investigate, for each combination of dose level and number of trials, the ability of both the model-based and jackknife estimates of standard error to describe the underlying variability in $\hat{\eta}_{j}(x)$. Specifically, for each panel in the plot, we present the averages of the standard errors $\sqrt{\hat{\mathbf{V}}_{\mathrm{MB}}\left(\hat{\eta}_{j}(x)\right)}$ and $\sqrt{\hat{\mathbf{V}}_{\mathrm{JK}}\left(\hat{\eta}_{j}(x)\right)}$. In this figure we also give the Monte Carlo standard error of estimating $\hat{\eta}_{j}(x)$ which serves to approximate the true variability in $\hat{\eta}_{j}(x)$. The Monte Carlo standard error is given by $\operatorname{se}_{\mathrm{MC}}\left(\hat{\eta}_{j}(x)\right)=\sqrt{\frac{1}{N-1} \sum_{m=1}^{N}\left(\hat{\eta}_{k}^{[m]}(x)-\overline{\hat{\eta}}_{k}(x)\right)^{2}}$ where $\hat{\eta}_{j}^{[m]}(x)$ is the estimate from the $m=1, \ldots, N$ simulated dataset and $\bar{\eta}_{j}(x)=\frac{1}{N} \sum_{m=1}^{N} \hat{\eta}_{j}^{[m]}(x)$ is their average. Both the model-based and the jackknife estimators display unusual behaviour with the smallest sample size in the factorial design: the model-based estimate is much larger than the Monte Carlo standard error near the two boundaries of the domain, but very comparable in the middle of the domain; the jackknife estimate is uniformly larger than the Monte Carlo standard error, and appears to increase with the covariate $x$. This last point is likely be due to the nature of the jackknife estimator and that integration of a positive function is a cumulative operator. However, much like the bias estimates these characteristics diminish and disappear with larger sample sizes.

For each simulated data set in a given design, we computed a $95 \%$ confidence interval for $\hat{\eta}_{j}(x)$ using both $\left.\sqrt{\hat{\mathbf{V}}_{\mathrm{MB}}\left(\hat{\eta}_{j}(x)\right.}\right)$ and $\left.\sqrt{\hat{\mathbf{V}}_{\mathrm{JK}}\left(\hat{\eta}_{j}(x)\right.}\right)$ and determined the proportion of times that each interval contained the true $\eta_{j}(x)$. These coverage rates are presented in Figure 2.9 and we observe that the coverage rates for all the designs are near the nominal level, however, across the domain of $x$ there appears to be more stability in the rates for larger sample sizes. 


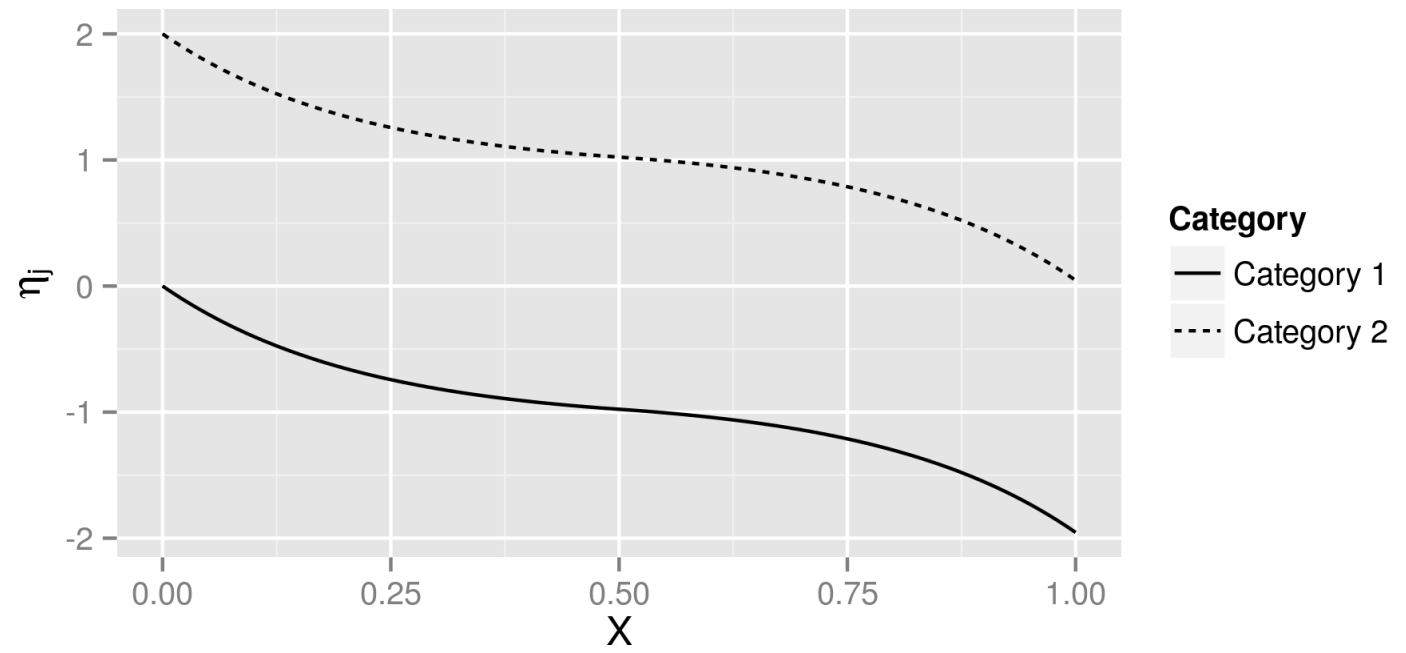

Figure 2.6: Plot of the true $\dot{\eta}_{j}(x),(j=1,2)$ used in the FXMS simulation. 


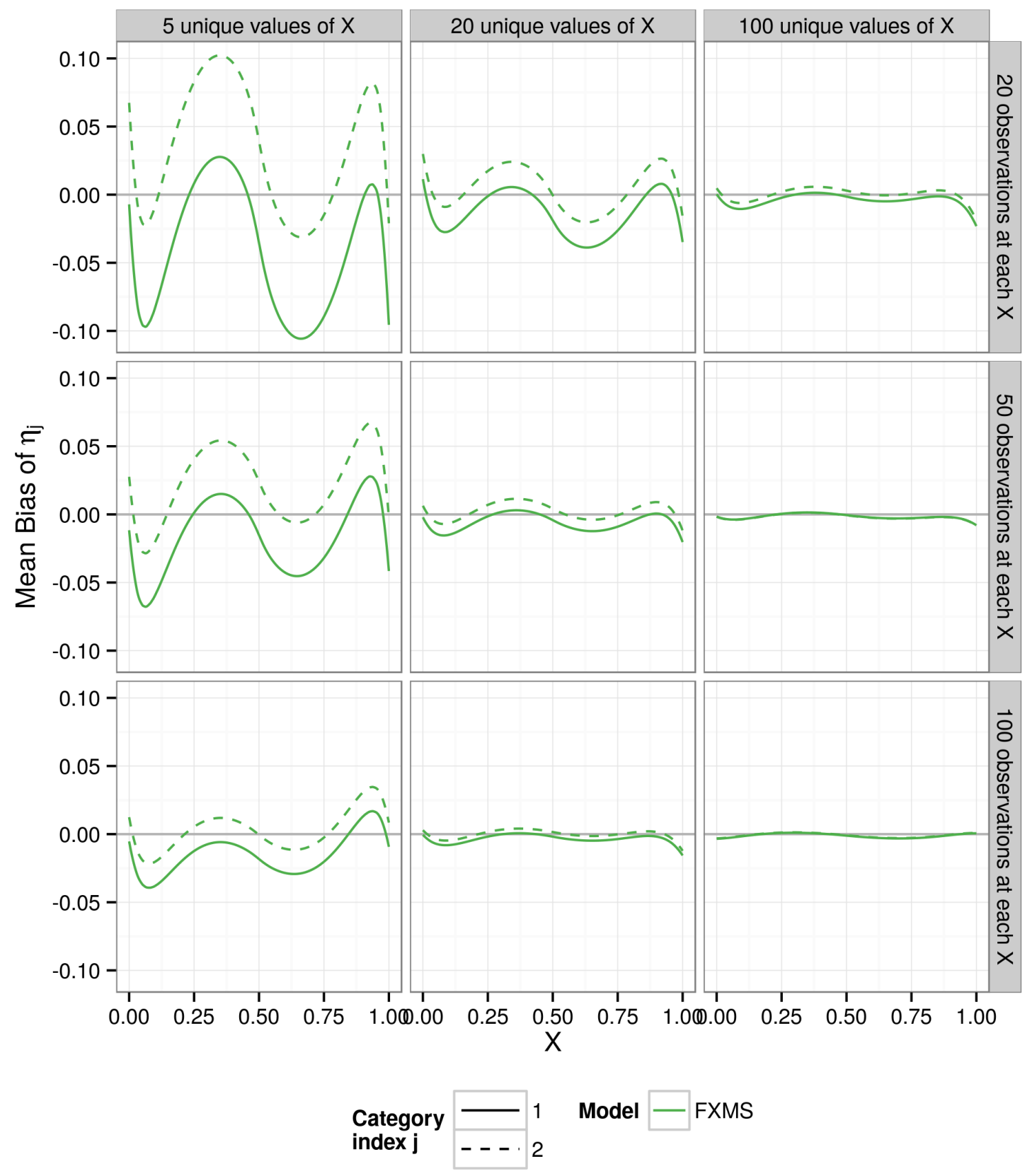

Figure 2.7: Estimated bias of $\hat{\eta}_{j}(x), j=1,2$, over 1,000 simulated replicates for the FXMS model and each of nine designs. 




Figure 2.8: Mean model-based and Jackknife standard errors of $\hat{\eta}_{j}(x)$, $j=1,2$, over 1,000 simulated replicates for the FXMS model and each of nine designs. The Monte Carlo standard error estimate is also displayed. 


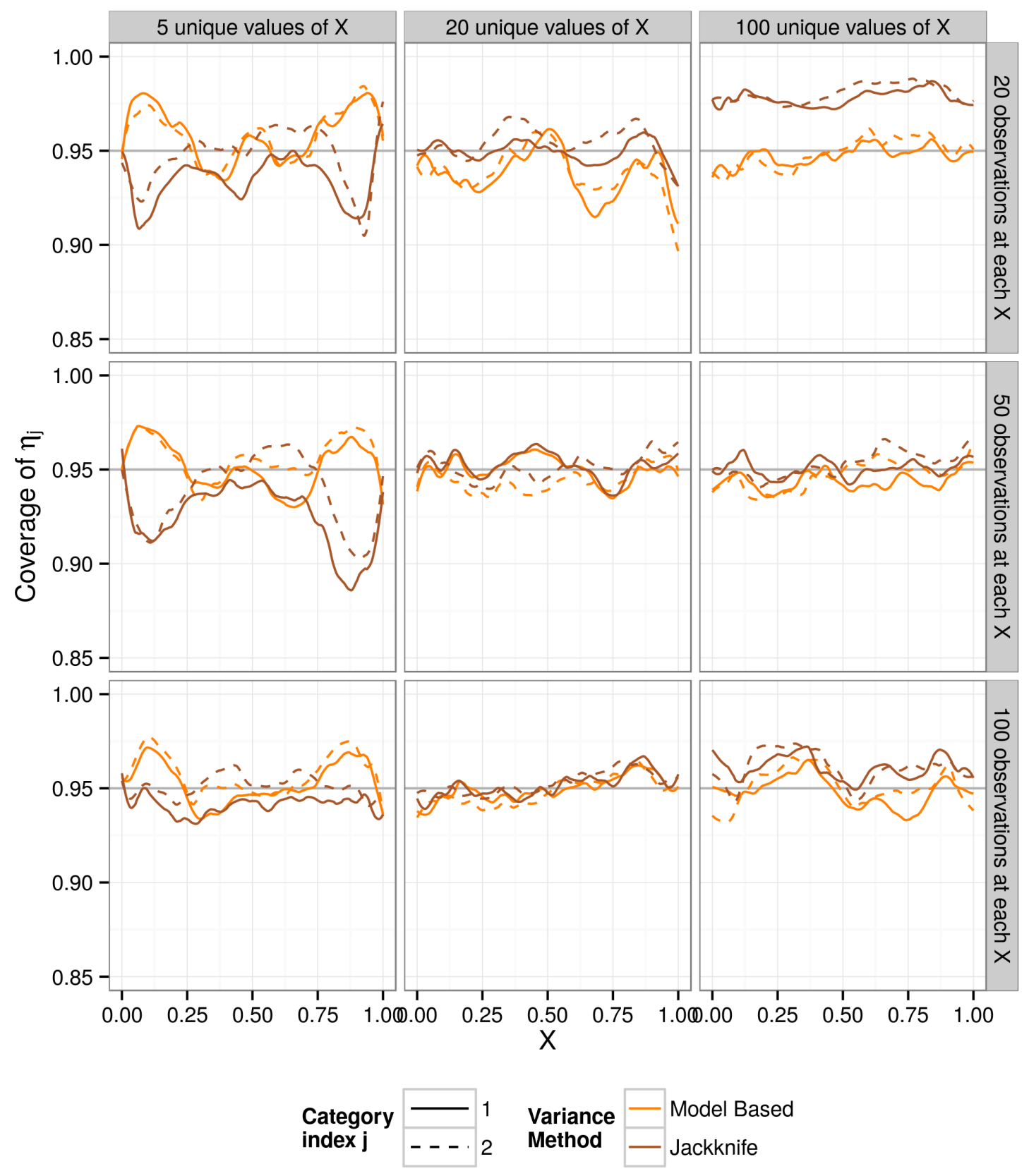

Figure 2.9: Model-based and Jackknife coverage rates of 95\% confidence intervals for $\eta_{j}(x), j=1,2$, over 1,000 simulated replicates for the FXMS model and each of nine designs. 
The second simulation investigates the PMS model. Here we specify the true model as in (2.21) with parameter values $\dot{\boldsymbol{\alpha}}=(0,2.0)^{\top}, \dot{\boldsymbol{\beta}}=\left(\dot{\beta}_{1}, \ldots, \dot{\beta}_{n_{k}}\right)^{\top}$ where $\dot{\beta}_{k}=10\left(t_{k}-0.5\right)^{2}+\log 0.5$, and $\lambda=5$. This value $\lambda$ in not used in generating data, however it is used (and held fixed) when estimating the parameters. We follow the guideline given in Section 2.3.2 to determine the number of knots to use in each design, and place these knots at equally spaced intervals, resulting in the knot sequence $\dot{\mathbf{t}}=\left\{t_{1}, \ldots, t_{K}\right\}$.

We follow an analogous procedure as with the FXMS simulation; only we replace the model-based estimates of the variance by $\hat{\mathbf{V}}_{\mathrm{PEN}}(\hat{\boldsymbol{\theta}})$, the penalized variance estimator in (2.25). The results of the PMS simulation are illustrated graphically in Figures 2.11, 2.12 and 2.13. Tabular summaries for both the FXMS and the PMS simulation are given in Appendix A.

In Figure 2.11 we observe that there is apparent bias in the estimates, especially with smaller samples sizes, however it does not entirely dissipate with the largest sample in the simulation. The three types of standard error estimates shown in Figure 2.12 are mostly in agreement with one another, displaying slightly more fluctuation at the smallest sample size. In Figure 2.13 we see that, for all designs, the coverage rates are not uniform across the domain of $x$; they appear to be best at the boundaries and the centre of the domain. The also show improvement in accuracy as the sample size increases and, with the largest design, they approach the nominal level across the domain. 


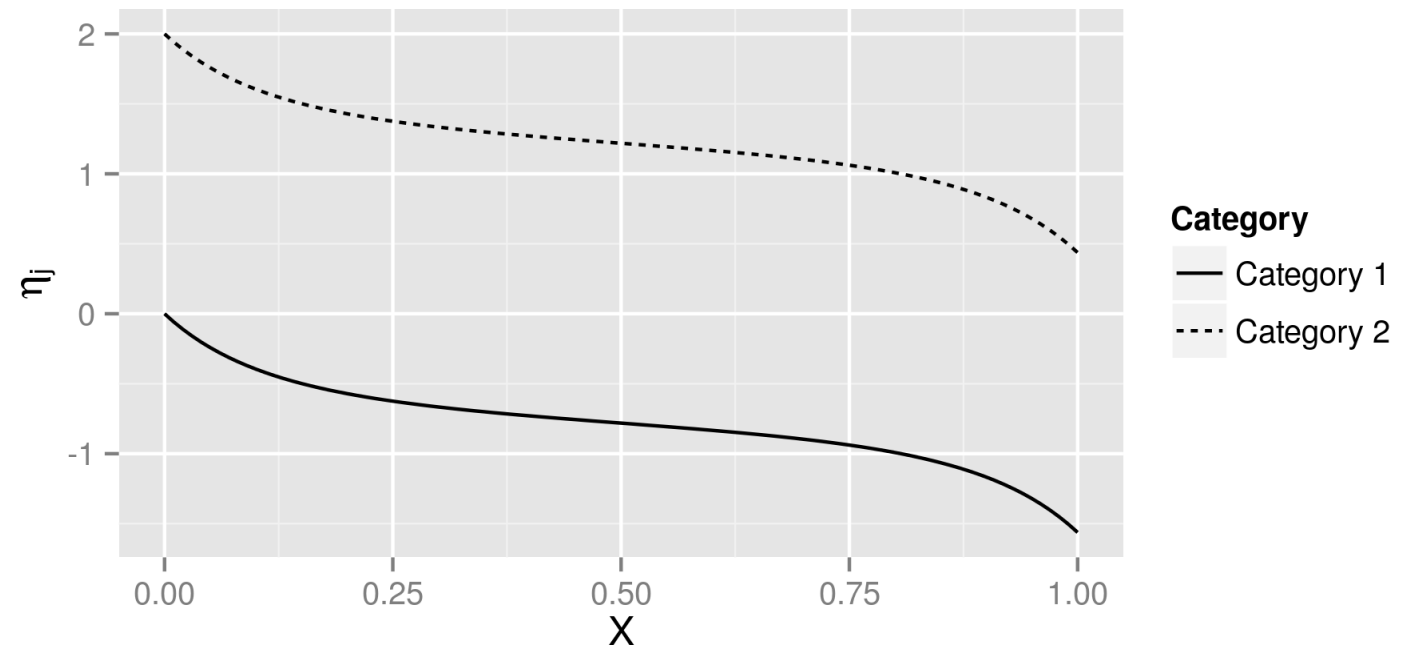

Figure 2.10: Plot of the true $\eta_{j}(x),(j=1,2)$ used in the PMS simulation. 


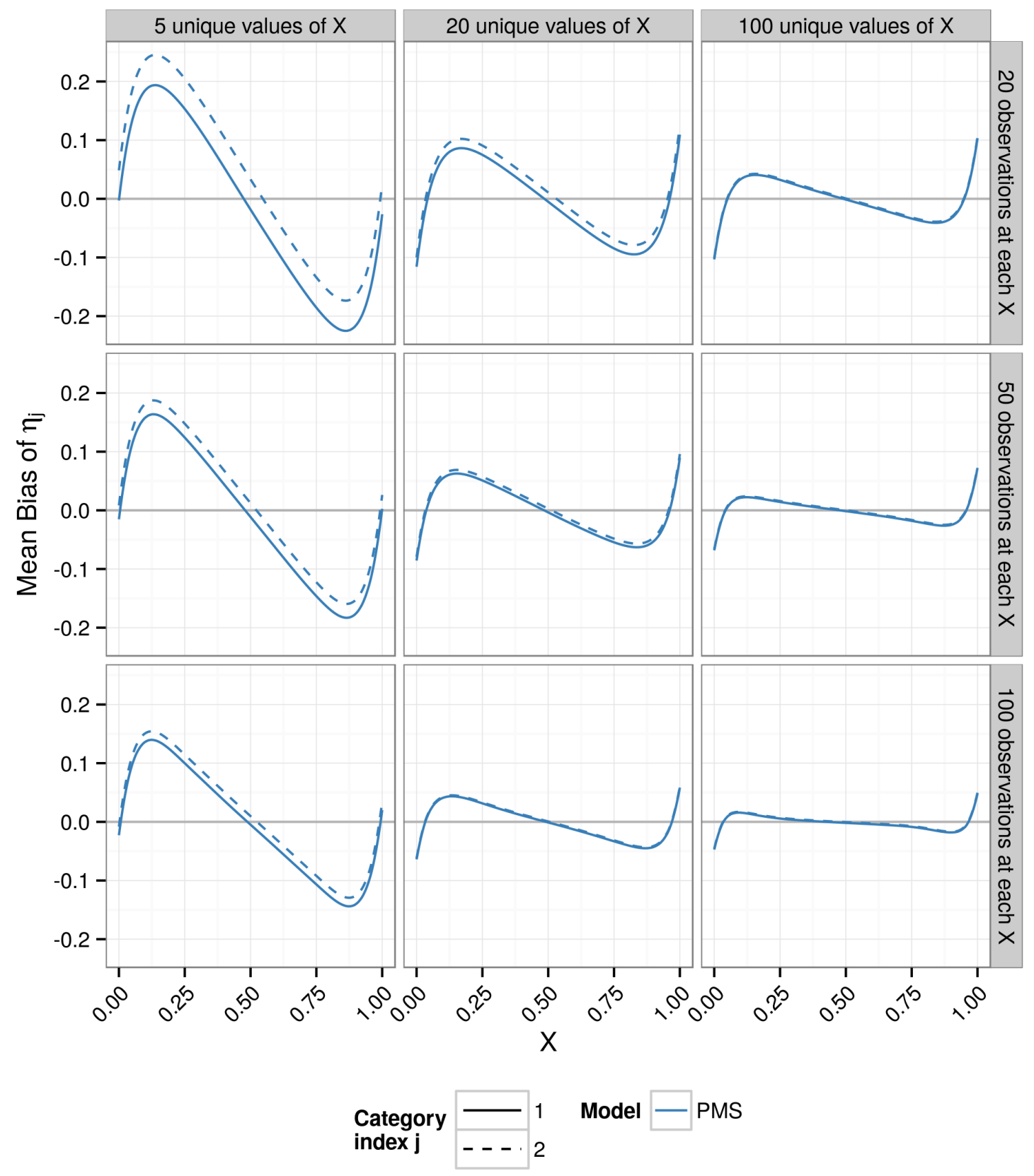

Figure 2.11: Estimated bias of $\hat{\eta}_{j}(x), j=1,2$, over 1,000 simulated replicates for the PMS model and each of nine designs. 


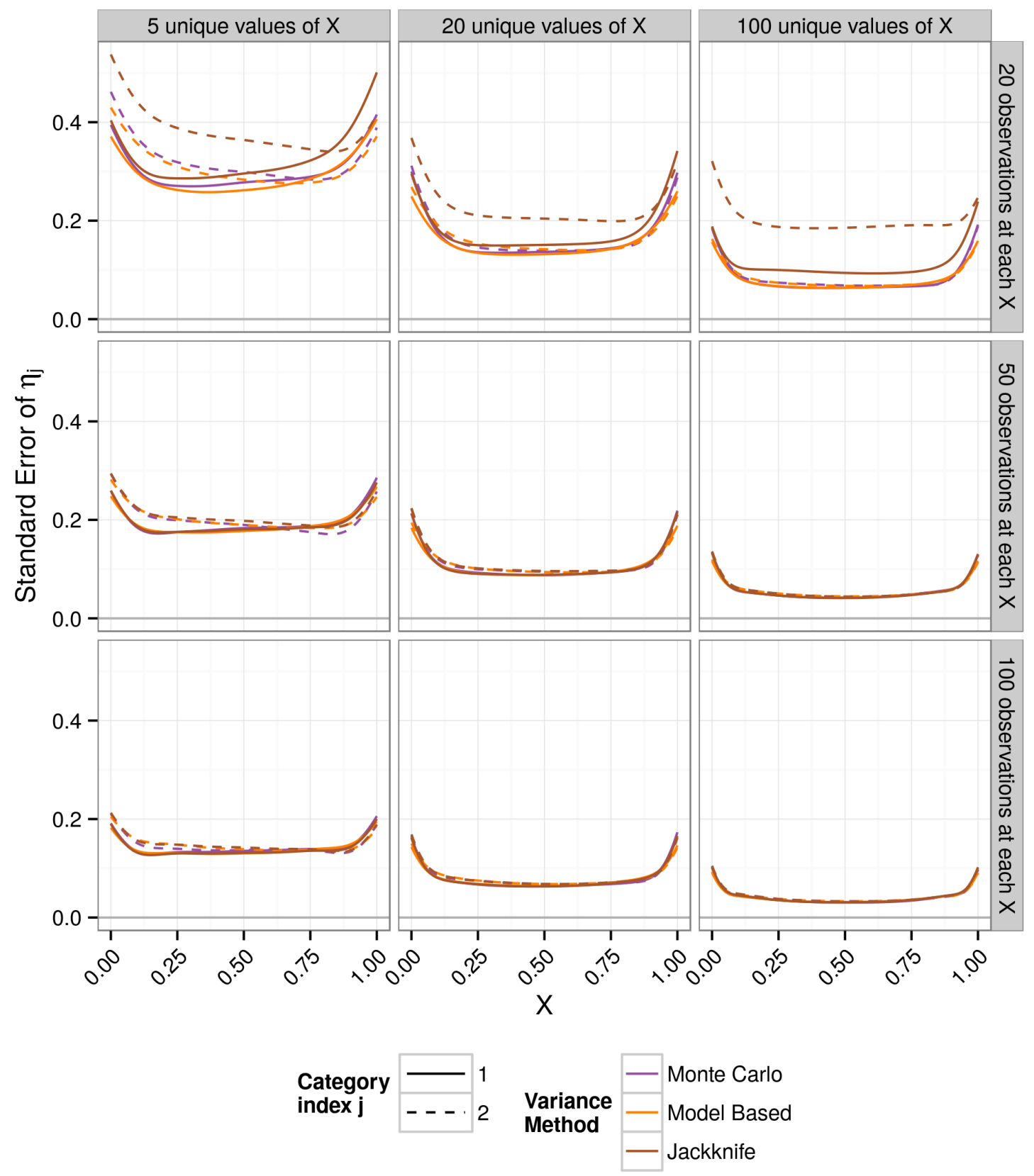

Figure 2.12: Mean model-based and Jackknife standard errors of $\hat{\eta}_{j}(x)$, $j=1,2$, over 1,000 simulated replicates for the PMS model and each of nine designs. The Monte Carlo standard error estimate is also displayed. 


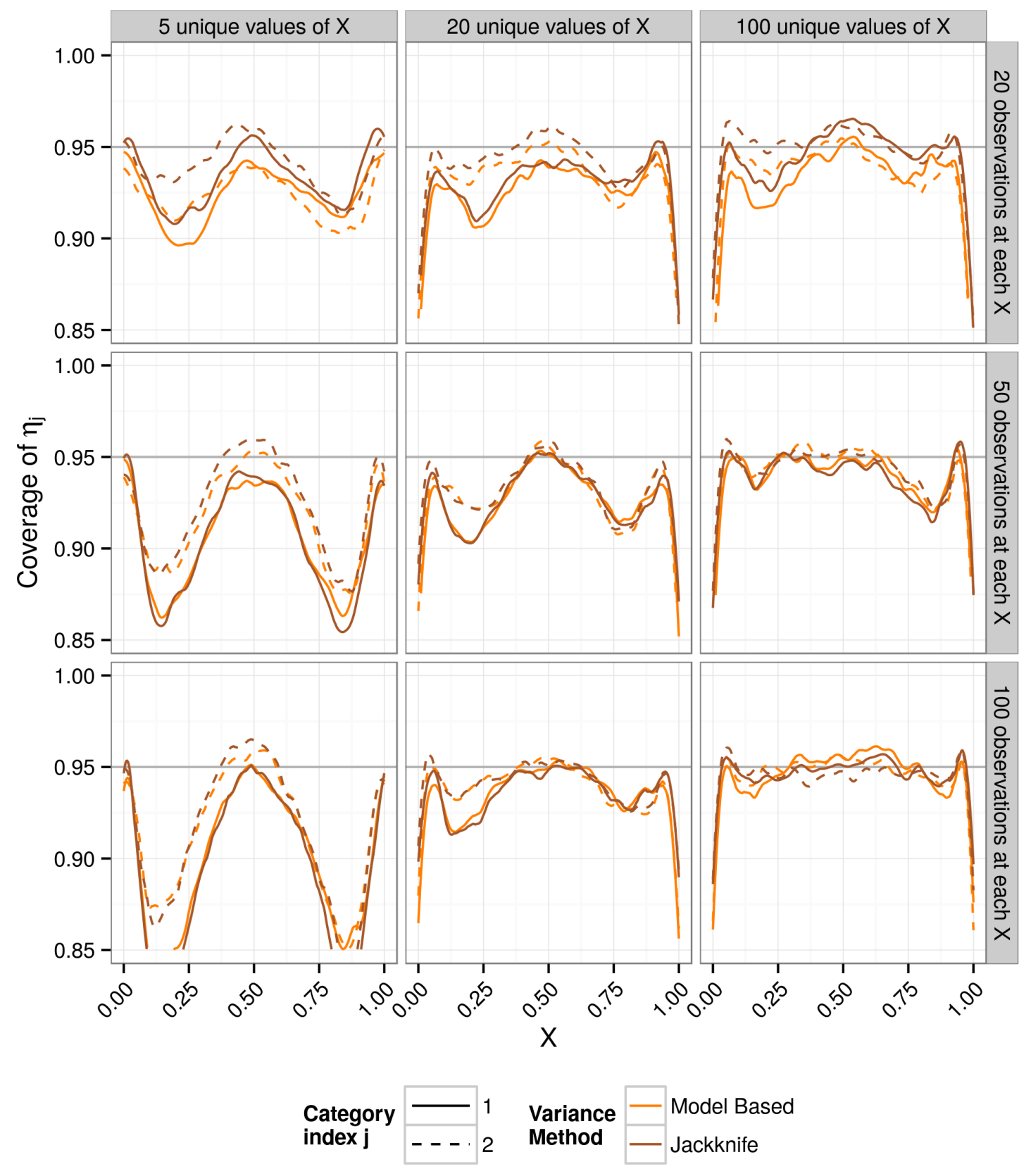

Figure 2.13: Model-based and Jackknife coverage rates of $95 \%$ confidence intervals for $\eta_{j}(x), j=1,2$, over 1,000 simulated replicates for the PMS model and each of nine designs. 


\subsection{Conclusion}

In this chapter we introduced monotone splines to the cumulative logit ordinal model and showed that they can provide a superior fit. We discussed two estimation methods, an adaptive fixed knot approach and a penalty-based approach, and also two standard error estimates, model-based and jackknife. We investigated both adaptive fixed knot and penalized splines, with the latter providing more flexibility. 


\section{Chapter 3}

\section{The Benchmark Dose for Ordinal Models}

\subsection{Introduction}

According to the United States Environmental Protection Agency (USEPA), doseresponse modeling for a particular chemical "involves an analysis of the relationship between exposure to the chemical and health-related outcomes" (USEPA, 2012). For dose-response analysis associated with health effects other than cancer, initial attempts to define a reference value were based on the lowest observed adverse effect level (LOAEL), or the no-observed-adverse-effect-level (NOAEL). The USEPA defines the LOAEL as "the lowest dose for a given chemical at which adverse effects have been detected," and the NOAEL as "the highest dose at which no adverse effects have been detected" (USEPA, 2012).

A number of limitations of the NOAEL/LOAEL approach have been identified; these include those cited in Crump (1984), Gaylor (1983), Kimmel and Gaylor (1988), Leisenring and Ryan (1992), and USEPA (1995). These limitations are nicely summarized in USEPA (2012). One of the major shortcomings of the NOAEL and the LOAEL is that they are constrained to be one of the dose levels used in the study 
under consideration. Thus not only are they highly susceptible to experimental design, but they are not readily comparable across different studies. Additionally, by not taking into account the variability of the design of an experiment, the approach has the rather undesirable property that experiments with smaller sample sizes often lead to higher NOAEL.

In an effort to address some of the limitations of the NOAEL/LOAEL approach, for quantal and continuous responses, Crump (1984) proposed the Benchmark Dose (BMD). Subsequently, for continuous responses, Crump (1995) proposed a definition of the BMD which builds upon Gaylor and Slikker (1990) and Kodell and West (1993). A number of authors (Crump (2002), Sand et al. (2008) and others) have referred to the method of Crump (1995) as the hybrid approach. The details of these methods are among those discussed in Section 3.2.

The BMD is now well-defined for quantal and continuous data, and is used commonly by a variety of organizations; including the USEPA (see USEPA (2012)). Relatively speaking, much less research has been directed towards defining a BMD for a response that is ordinal in nature. There have, however, been a few studies focusing on this problem of late, including Regan and Catalano (2000), Faes et al. (2004), and Chen and Chen (2014).

In this chapter, we propose an alternative method for defining the BMD for ordinal outcome data. Generally speaking, we wish to find an approach that will be robust to the number of ordinal categories into which we divide the response. In Section 3.2, we begin by presenting the definitions of the BMD proposed by Crump (1984) for quantal and continuous responses, and subsequent revision of the latter by Crump (1995). We also include a review of the existing methods for proposing a BMD for ordinal, multinomial and mixed multivariate responses. In Section 3.3, we propose 
an alternative approach for determining the BMD for ordinal outcome responses. In Section 3.4 we present two methods of evaluating a confidence limit for the BMD. We investigate the performance of the BMD proposed in Section 3.3 and the associated confidence limits via a simulation study in Section 3.5. Conclusions and discussion are provided in Section 3.6.

\subsection{Definitions of the Benchmark Dose}

There is a strong need for a reference dose standard in toxicological risk assessment to ensure that any regulatory decisions are scientifically sound. Of the alternatives, the USEPA prefers the BMD and provides Benchmark Dose Software (BMDS) for practitioners to use. This software can fit a wide variety of models (USEPA, 2012). In Europe, use of the software PROAST (RIVM, 2012) to calculate the BMD is more common. This software was developed for and is available from the National Institute for Public Health and Environment of the Netherlands (RIVM). The European Food Safety Agency (EFSA) has recommended to use the BMD but has not mandated its use (EFSA, 2009).

We now discuss the development of the BMD as a reference dose. Following the standard notation in BMD literature, we let $d$ denote the covariate corresponding to dose level. Suppose we have a response outcome and a dose variable for each of $n$ subjects. We denote the dose variable for subject $i$ by $d_{i}$, and the response by $Y_{i}$, $i=1, \ldots, n$. In addition, we let $X=\left(d_{1}, \ldots, d_{n}\right)$ be the vector of doses for all the subjects. We also suppose the mean of $Y$ is some known monotone function and $\boldsymbol{\theta}$ is 
a vector of unknown model parameters. We define two methods of calculating risk,

$$
\begin{aligned}
\text { Additional Risk: } R & =p(d)-p(0) \\
\text { Extra Risk: } R & =\frac{p(d)-p(0)}{1-p(0)}
\end{aligned}
$$

where $p$ is some probability function of dose $d$. In the BMD definitions that follow, $p(d)$, represents the probability of adverse effect at dose $d$. Note that the extra risk is the additional risk relative to that for the control group dose $d=0$.

\subsubsection{Quantal Response}

Suppose that the response $Y$ is quantal, and denote the two values it can obtain by $\{1,2\}$. (We adopt this notation instead of the more commonly-used $\{0,1\}$ to be consistent with the notation for ordinal variables that is presented in this thesis.) We also imagine that we have a model

$$
P(Y=2 \mid X=d)=H(d),
$$

where $H(d)$ is some known monotone function. Crump (1984) defined the benchmark dose (BMD) for a quantal outcome as the change in the response of an adverse effect for some specified value of $R$. Typically, $R=0.01,0.05$, or 0.10 . Note that monotonicity is a requirement for determining the BMD since the inverse of $p(d)$ in (3.1) is needed. With quantal outcomes the probability of response is taken to be equivalent to the probability of adverse effect; hence the estimate for $p$ comes directly from the model fit. The BMD can then be calculated directly from the risk in (3.1) using the model estimates. 


\subsubsection{Continuous Response}

A workshop dedicated to the BMD concept was held while the topic was still in its infancy; among the issues discussed was the challenge in defining a BMD for non-quantal data (see Barnes et al., 1995). The most common type of non-quantal response found in practice are those which are of continuous form. Generally speaking, continuous data contains much more rich and varied information than quantal data, however, for this very reason defining a BMD for continuous response is more complex. Fundamentally, the main issues are determining what measures to use for the location and scale of the data, and how to adjust for them so the resulting BMD is robust. Another key concern is consistency with the quantal definition.

To date there is still disagreement as to the best method for defining the BMD for continuous data, however, most of the definitions in common use are relatively minor variations of one another. In their reference document on the BMD topic, the USEPA suggests that all publications on BMD include an estimate calculated with one standard deviation from the mean, regardless of whether it is used for analysis. This ensures that results are comparable across studies (USEPA, 2012).

In this section we take $Y$ to be a continuous response and let $\mu(d)=\mathrm{E}(Y \mid X=d)$, which can be estimated from some model.

\section{Definitions Not Reliant upon the Adverse Effect}

A number of authors have proposed definitions of the BMD for continuous response that are not reliant upon the adverse effect. First, note that the additional risk in (3.1) for a quantal response is just the difference in the mean at dose $d$ from the mean of the control group. Thus, if we let $\mu(d)$ be the mean response at dose $d$ for a continuous response of interest, then the analogous expression to (3.1) is $\mu(d)-\mu(0)$. 
Since this term is location invariant but not scale invariant, Crump (1984) suggests scaling it by the response at control $d=0$ to get

$$
\frac{|\mu(d)-\mu(0)|}{\mu(0)},
$$

an expression he refers to as the 'extra response'. He based the first definition of the continuous BMD on this expression; he proposed the continuous BMD be the dose, $d$, corresponding to a specified increase in the extra response. He also indicates that standardization of the scale could be achieved by other quantities, and in particular mentions scaling by the standard deviation at the control $d=0$,

$$
\frac{|\mu(d)-\mu(0)|}{\sigma(0)} .
$$

Murrell et al. (1998) argued against (3.3) because it does not entirely remove the effect of the background level of response. They proposed instead to scale by the dynamic range of the response,

$$
\frac{|\mu(d)-\mu(0)|}{\mu_{\max }-\mu(0)},
$$

considering (3.5) more consistent with the definition for the BMD with quantal response since it is completely standardized with respect to scale. They noted that, for quantal responses, $\mu$ is a proportion and $\mu_{\max }=1$, so (3.5) is equivalent to the extra risk specification in (3.1).

Slob and Pieters (1998) argued against (3.4) because $\sigma(0)$ could lead to estimates which were too variable with small sample sizes and also that $\sigma(0)$ may not be representative of the scale of the response distribution when $d>0$. They defined the 
critical effect size (CES) along with the corresponding critical effect dose (CED), and suggested that the complete uncertainty distribution of the CED be used instead of just the BMD. The definition of the CED is such that it is equivalent to (3.3). The CES/CED concept is developed further in Slob (2002).

\section{Definitions Reliant upon the Adverse Effect}

In addition to the above, other studies have suggested definitions that are reliant upon the adverse effect. In this regard we summarize the work of Crump (1995). Crump (1995) commented that (3.3) and (3.4) are both fundamentally different from the BMD used with quantal responses; since they are based on the mean response $\mu$ they cannot be interpreted as if they were based on probabilities. To obtain comparable estimates from continuous data as with quantal data, one option is to dichotomize the continuous response at some cutoff and fit a quantal model. Both Allen et al. (1994) and Gaylor (1996) advise against this because of a loss of power.

Crump (1995) utilized components of Gaylor and Slikker (1990) and Kodell and West (1993) to suggest a new BMD definition for continuous response. Specifically, he assumed that at dose $d$, the cumulative distribution function of the response, $F_{d}$, is known. He then defined the proportion of the distribution above the cutoff value, $k$, as the function

$$
p(d)=1-F_{d}(k)
$$

The cutoff value is the background probability of adverse effect and must be chosen suitably. With no prior information about the nature of the distribution $F_{d}$, one can use a value for $k$ which is 2 or 3 standard deviations above the background mean. 
Alternatively, $k$ can be set to correspond to some percentile of the distribution at the control, for example $1-F_{0}(k)=0.05$. Equation (3.6) can be interpreted as the probability of adverse effect for continuous data. It can be used in one of the risk functions in (3.1) to evaluate the BMD. This method of calculating the BMD for continuous data has become known as the hybrid approach.

If we can assume that the continuous response is normal with constant variance over doses then

$$
p(d)=\Phi\left[\frac{k-\mu(d)}{\sigma}\right],
$$

where $\Phi$ is the cumulative normal distribution, $\mu(d)$ is the mean response at dose $d$ and $k$ is some cutoff value. The value of $k$ is used to dichotomize the data and then the BMD is computed on the resulting quantal data.

One could view the cutoff value $k$ as serving to dichotomize the data, thereby providing an equivalency to the quantal data situation. We represent this graphically in Figure 3.1. Thus, the same value of BMD would result from (3.1) regardless of whether (3.2) or (3.7) is used since these two expressions are equivalent by construction.

\subsubsection{Non-Quantal, Non-Continuous Responses}

In this section we give a brief overview of some of the ways a BMD has been determined for non-quantal, non-continuous responses. Outcomes considered here include univariate ordinal, multinomial, and mixed multivariate with a combination of quantal, ordinal or continuous responses. All of the methods in this section employ the adverse effect concept. 


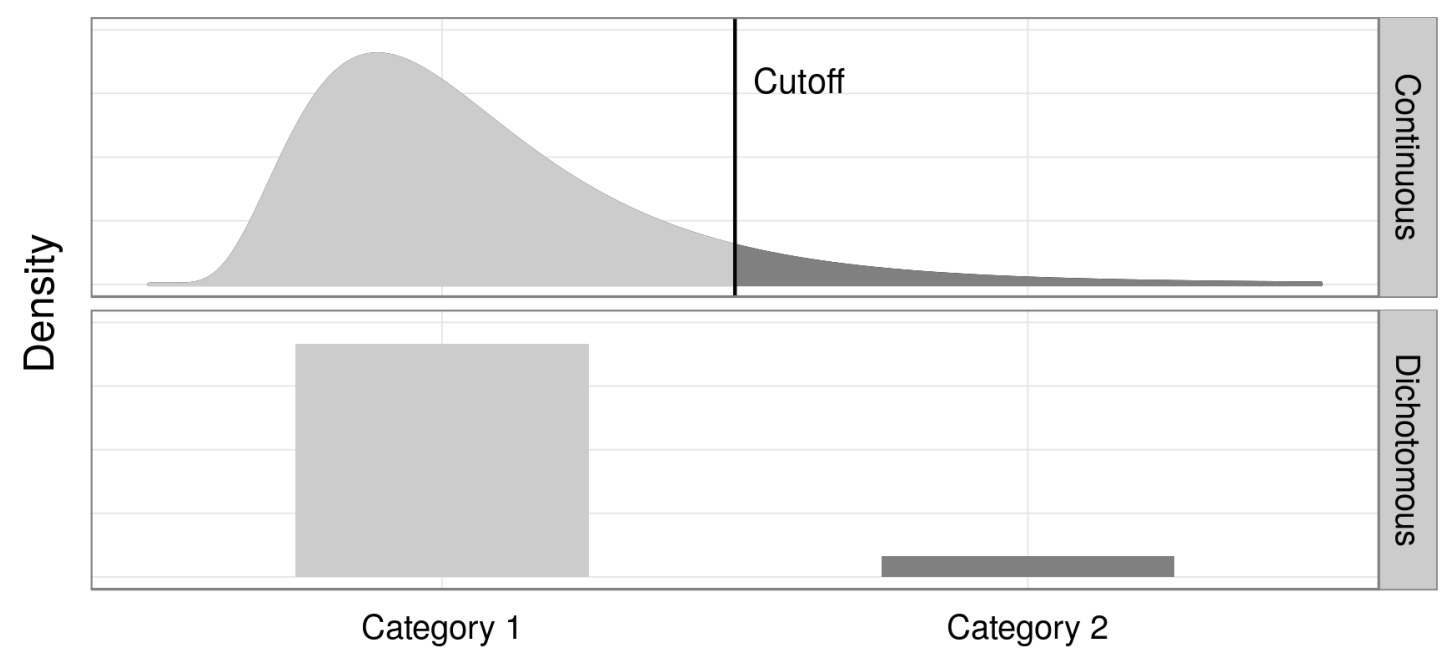

Figure 3.1: Dichotomizing a continuous variable to a quantal variable.

The first group of models define the probability function,

$$
p(d)=P(\text { any outcome is adverse } \mid X=d),
$$

as an overall adverse effect. This definition of adverse effect has been suggested on several occasions; by Slob and Pieters (1998) for univariate ordinal data, Krewski and Zhu (1994) for multinomial response, Gaylor et al. (1998) for a multivariate mix of quantal and continuous responses, and Regan and Catalano (2000) for a mix of two ordinal outcomes and a continuous response.

Faes et al. (2004) fit a bivariate ordinal and continuous model using this definition for the marginal distribution of $Y$. In their model, the full distribution of the response is $p(d)=P(Y>j$ or $Z<k \mid X=d), j=1, \ldots, C-1$ for some cutoff $k$ corresponding to the continuous outcome $Z$. Mbah et al. (2014) fit a latent class model with two classes to multivariate binary data. The resulting model fit is $p(d)=p(d ; z)$ where $z$ 
is the latent continuous outcome.

Alternatively, ordinal data can be dichotomized at each of the $C-1$ severity levels into adverse or not adverse. In this approach the probability of adverse response for the $j$-th category, $j=1, \ldots, C-1$, is given by

$$
p(d)=P(Y>j \mid X=d) .
$$

Moerbeek et al. (2004) follow this approach and choose to keep all $C-1$ probabilities. Presumably, the implication of this decision is that a total of $C-1$ different values of BMD can be determined, one from each $p_{j}(d)$. We shall henceforth refer to these BMD values as $\mathrm{BMD}_{j}, j=1, \ldots, C-1$. Unfortunately, the definition of a reference dose when multiple values of BMD are available requires some thought. By contrast, the USEPA declare that a single severity level from the $C-1$ possibilities should be chosen (USEPA, 2012). However, in this case the choice of severity level at which to split the response is somewhat arbitrary and requires input from the practitioner.

\subsubsection{Ordinal Response as Proposed by Chen and Chen}

Chen and Chen (2014) propose an approach to BMD calculations for ordinal data that stems from the hybrid approach for continuous data. We shall henceforth refer to their method as CCBMD. They assume that the ordinal data has an underlying normal distribution, and the transition between each category has a cutoff value $z_{j}$, $j=1, \ldots, C-1$.

Chen and Chen (2014) then calculate the hybrid BMD by first finding the $C-1$ cutoff values $\left(\mathrm{BMD}_{j}\right)$. In this sense, the approach to obtain these $\mathrm{BMD}_{j}$ values can simply be viewed as an extension of the illustration above that is demonstrated in 
Figure 3.1. Note that for the two category case, there is equivalency between the hybrid approach for continuous outcomes and with the quantal response situation. Figure 3.2 presents an example of the approach underlying the method proposed by Chen and Chen (2014) for the case of $C=4$ categories that would produce three BMD values using cutoffs $z_{1}, z_{2}$ and $z_{3}$.


Figure 3.2: Categorizing a continuous variable to an ordinal variable.

We remarked earlier about the challenge of determining a single reference dose when multiple BMD values exist. In order to arrive at a single BMD value to serve as the reference dose, Chen and Chen (2014) used a weighted sum of the $\mathrm{BMD}_{j}$ values, namely

$$
B M D=\sum_{j=1}^{C-1} \omega_{j} \cdot \mathrm{BMD}_{j}
$$

where $\sum_{j=1}^{C-1} \omega_{j}=1$. The $\omega_{j}$ are determined by specified loss functions. Chen 
and Chen (2014) provide suggested functions for $C=3,4$, and 5. Since the resulting CCBMD value can change depending on how these functions are defined, Chen and Chen (2014) also give general advice on the characteristics they should display.

The definition of CCBMD is only consistent with the quantal method when a probit model is fit to the data. It cannot be compared with the hybrid method, as for $C>5$ there are an infinite number of ways that the loss functions can be defined.

One of the issues worthy of note concerning the approach of Chen and Chen (2014) is the robustness of the BMD value with respect to the number and location of cutoff points. For example, imagine a study where the decision was to categorize the ordinal response of interest into one of four categories. Alternatively, the same investigation might have been conducted by choosing only three categories for the response. Given that the outcomes are tied to the same distribution it would seem reasonable to argue that, ideally, the value of the BMD should not be affected. We demonstrated in the right panels of Figure 3.3 that this is not the case for CCBMD, and that the differences can be substantial.

Specifically, the right panels display the true CCBMD values for a BMR of $R=0.10$ where the true distribution follows a probit model. The top right panel shows the CCBMD with $C=3$, while the bottom right panel shows the result with $C=4$ categories, where the last category has a small conditional probability, namely, $P(Y=4 \mid X=0)=0.01$. Note that this lower panel represents a situation where the vast majority of responses occur within the first three categories, $P(Y \leq 3 \mid X=0)=$ 0.99 , which is comparable to $P(Y \leq 3 \mid X=0)=1$ in the top panel.

In the next section we propose an alternative method that makes the BMD value more robust to changes in the number and location of cutoff values. 




Figure 3.3: $\mathrm{BMD}$ values for $\mathrm{BMR}=0.1$ where the true distribution follows a probit model with $\beta=-1 / 15$. OBMD and CCBMD appear in the left and right panels respectively; the top panels show the values when $C=3$, while the bottom panels show the case where $C=4$ and $P(Y=4 \mid X=0)=0.01$. We remark that $P(Y \leq 3 \mid X=0)=1$ in the top panels and that $P(Y \leq 3 \mid X=0)=0.99$ in the bottom panels. 


\subsection{Proposed Benchmark Dose for Ordinal Response}

Our proposed approach uses the risk equation (3.1) in the same manner as the quantal and continuous BMD definitions. To do so we define a probability of adverse response for ordinal data. It is designed to be robust and dose not require practitioner input.

\subsubsection{Motivation}

One of the impetuses for the continuous data hybrid method of Crump (1995) is consistency in BMD estimates between continuous and quantal data derived from the same source. Conceptually, any true underlying mechanism governing a doseresponse process of interest inherently possesses a unique BMD value. Ideally, our ability to estimate the BMD should not be affected by how we observe the process. For example, if the mechanism is of a continuous nature, then whether we record our observations as continuous data or as ordinal data we are still estimating the same true quantity. Likewise, the number of ordinal categories we choose to observe should be irrelevant.

With this in mind, we propose a method for calculating the BMD for ordinal data which explicitly endeavours to estimate the BMD in a manner consistent with both the quantal BMD and the hybrid method for continuous response. Indeed, when $C=2$, the proposed method reduces to the quantal method, and (under mild conditions) when $C \rightarrow \infty$ the method results in the same estimates as the hybrid method. 


\subsubsection{Details}

In the case of quantal data, the two categories represent a dichotomy; an adverse response is either present or absent. With ordinal data, the concept of adverse response is more nuanced with categories that can represent a position between the two extremes, or a partially adverse response. For each category, to distinguish the effect of exposure at the level associated with that category on the adverse response, we consider the conditional probability $p_{j}=P($ Adverse Response $\mid Y=j)$. We will assume that $p_{j}$ is independent of dose; that is, a response observed to be from category $j$ is interpreted identically, regardless of the dosage received. To this end we adopt the behaviour at $d=0$ as a reference point, and will make use of $\tau_{0 j}, \pi_{0 j}, v_{0 j}$ where $\tau_{d j}=P(Y<j \mid X=d), \pi_{d j}=P(Y=j \mid X=d)$ and $v_{d j}=P(Y>j \mid X=d)$ for all $j=1, \ldots, C$.

Using these conditional probabilities, we can write the probability of an adverse response at dose $d$ as

$$
\begin{aligned}
p(d) & =P(\text { Adverse Response } \mid X=d) \\
& =\sum_{j=1}^{C} P(\text { Adverse Response } \mid Y=j, X=d) P(Y=j \mid X=d) \\
& =\sum_{j=1}^{C} P(\text { Adverse Response } \mid Y=j) P(Y=j \mid X=d) \\
& =\sum_{j=1}^{C} p_{j} \pi_{d j} .
\end{aligned}
$$

We employ some general assumptions in order to infer the relationship between the categories and $p_{j}$. A natural extension of adverse response for quantal to ordinal categories is that the $p_{j}$ 's are ordered, and the values associated with the first and last 
categories are 0 and 1 respectively, $0=p_{1} \leq p_{2} \leq \cdots \leq p_{C}=1$. We assume that $p_{j}$ lies somewhere between $\tau_{0 j}$ and $\tau_{0 j}+\pi_{0 j}$ and that it depends only on the probability of adverse response of those categories below it, $\tau_{0 j}$, and the probability of adverse response of those categories above it, $v_{0 j}$. Note that since $\pi_{0 j}=1-\tau_{0 j}-v_{0 j}, p_{j}$ can also be thought to depend on $\pi_{0 j}$. We now write $p_{j}$ as a weighted average of $\tau_{0 j}$ and $\tau_{0 j}+\pi_{0 j}$,

$$
\begin{aligned}
p_{j} & =\tau_{0 j}\left(1-w_{j}\right)+\left(\tau_{0 j}+\pi_{0 j}\right) w_{j} \\
& =\tau_{0 j}+\pi_{0 j} w_{j}
\end{aligned}
$$

where $w_{j}=w\left(\tau_{0 j}, \pi_{0 j}, v_{0 j}\right)$ for some function $w$ having range $[0,1]$. Next, we consider how $w_{j}$ should relate to each of the three probabilities $\tau_{0 j}, \pi_{0 j}$ and $v_{0 j}$.

Firstly we look at $\tau_{0 j}$. By considering the first category, $j=1$, we see that $P(Y<1 \mid X=0)=\tau_{01}=0$. Since $p_{1}=0$, we must have $w\left(0, \pi_{0 j}, v_{0 j}\right)=0$ for any $j$. As both $\tau_{0 j}>0$ and $w_{j}>0$, we have that $w_{j}$ should increase with

$$
P(Y<j \mid X=0)=\tau_{0 j}
$$

We take a similar approach for $v_{0 j}$. By considering the last category we see that $v_{0 C}=0$ and $p_{C}=\tau_{0 C}+\pi_{0 C} w_{0 C}=\tau_{0 C}+\pi_{0 C}+v_{0 C}=1$, thus $w\left(\tau_{0 C}, \pi_{0 C}, v_{0 C}\right)=1$. More generally for any $j$ with $v_{0 j}=0$, we have $w\left(\tau_{0 j}, \pi_{0 j}, 0\right)=1$. Thus as $v_{0 j}$ increases, $w_{j}$ should decrease, or equivalently $w_{j}$ increases with

$$
P(Y \leq j \mid X=0)=1-v_{0 j}=\tau_{0 j}+\pi_{0 j} .
$$

Finally we consider $\pi_{0 j}$ and note that we are concerned not with its absolute size, 
but its size relative to the other categories. If the probability

$$
P(Y=j \mid Y \geq j, X=0)=\frac{\pi_{0 j}}{\pi_{0 j}+v_{0 j}}
$$

is close to 1 then $v_{0 j}$ is negligible, as is the affect of categories greater than $j$; thus $j$ can effectively be treated as the last category and $w_{j}$ should be near 1 . Similarly, large values of $P(Y=j \mid Y \leq j, X=0)=\pi_{0 j} /\left(\tau_{0 j}+\pi_{0 j}\right)$ result in categories smaller than $j$ being negligible and small $w_{j}$. When its complement

$$
P(Y<j \mid Y \leq j, X=0)=\frac{\tau_{0 j}}{\tau_{0 j}+\pi_{0 j}}
$$

is small, $w_{j}$ will be small as well.

In the above we have seen four probabilities that are positively associated with $w_{j}$ : $P(Y<j \mid X=0)$ and $P(Y \leq j \mid X=0)$ which are not conditional on the value of $Y$, and $P(Y<j \mid Y \leq j, X=0)$ and $P(Y=j \mid Y \geq j, X=0)$ which are conditioned on the category value. We now consider the product of these four probabilities:

$$
\begin{aligned}
& P(Y<j \mid X=0) \cdot P(Y \leq j \mid X=0) \cdot P(Y<j \mid Y \leq j, X=0) \cdot P(Y=j \mid Y \geq j, X=0) \\
& =\tau_{0 j}\left(\tau_{0 j}+\pi_{0 j}\right)\left(\frac{\tau_{0 j}}{\tau_{0 j}+\pi_{0 j}}\right)\left(\frac{\pi_{0 j}}{\pi_{0 j}+v_{0 j}}\right) \\
& =\frac{\tau_{0 j}^{2} \pi_{0 j}}{\pi_{0 j}+v_{0 j}} .
\end{aligned}
$$

When $v_{0 j}=0$ the value of the expression in (3.11) is $\tau_{0 j}^{2}$. Recall however, that we require $w_{j}=w\left(\tau_{0 j}, \pi_{0 j}, 0\right)=1$. If we scale $(3.11)$ by a factor of $\left(\frac{1}{\tau_{0 j}+v_{0 j}}\right)^{2}$ this 
condition is satisfied. Thus we set

$$
w_{j}=\left(\frac{\tau_{0 j}}{\tau_{0 j}+v_{0 j}}\right)^{2} \frac{\pi_{0 j}}{\pi_{0 j}+v_{0 j}},
$$

which we substitute into (3.10) and simplify to get

$$
\begin{aligned}
p_{j} & =\tau_{0 j}+\pi_{0 j}\left(\frac{\tau_{0 j}}{\tau_{0 j}+v_{0 j}}\right)^{2} \frac{\pi_{0 j}}{\pi_{0 j}+v_{0 j}} \\
& =\frac{\tau_{0 j}\left[\tau_{0 j}\left(1-\tau_{0 j}\right)+v_{0 j}^{2}\right]}{\left(\tau_{0 j}+v_{0 j}\right)^{2}\left(1-\tau_{0 j}\right)}
\end{aligned}
$$

which we subsequently substitute into (3.9) to get

$$
p(d)=\sum_{j=1}^{C} \pi_{d j} \frac{\tau_{0 j}\left[\tau_{0 j}\left(1-\tau_{0 j}\right)+v_{0 j}^{2}\right]}{\left(\tau_{0 j}+v_{0 j}\right)^{2}\left(1-\tau_{0 j}\right)} .
$$

We propose a measure that we shall henceforth refer to as the Ordinal Benchmark Dose, or OBMD, which is obtained from the probability of adverse response in (3.12) in conjunction with the equation for additional risk, (3.1). The OBMD proposed here has several attractive characteristics. First, this measure is defined for any number of categories, without the need for user-dependent input. Secondly, if the probability of the ordinal response for any one of the $j$ categories is 0 for the control group, the OBMD is the same as when evaluated with one fewer category. Finally, we demonstrate through the left hand panel of Figure 3.3 that, when compared to the CCBMD proposed by Chen and Chen (2014) (presented in the right panel), that the OBMD is significantly more robust to the number of categories into which the ordinal response is divided. 


\subsection{Calculating the Lower Confidence Limit of the Benchmark Dose (BMDL)}

An important complement to the BMD is the lower confidence limit of the BMD, which we will refer to as the BMDL. Various methods have been proposed for calculating this limit, see Sand et al. (2006) for a good overview. In addition to the delta method and likelihood-based confidence intervals which we present below, he discusses bootstrap confidence intervals.

The BMDL is a one-sided confidence interval that provides a lower bound on the BMD, and gives an idea of the variability in the BMD point estimate. We consider two approaches to calculating the BMDL; the first uses the delta method and Wald statistic, while the second is based upon the likelihood ratio test.

\subsubsection{Delta Method Using the Wald Statistic}

Here we construct a confidence interval using the delta method and the Wald statistic which we presented in Section 2.2.4 of the previous chapter. However, instead of a two-sided interval, we construct a one-sided interval from the Wald statistic. In the

present case, we let $h(\hat{\boldsymbol{\theta}})$ refer to the estimator of BMD. We use (2.19) to construct a $100(1-\alpha) \%$ one-sided confidence interval for $h(\boldsymbol{\theta})$, the lower bound of which is

$$
b_{d}=h(\hat{\boldsymbol{\theta}})-z_{\alpha} \sqrt{\hat{V}(h(\hat{\boldsymbol{\theta}}))}
$$

where $z_{\alpha}$ is the $100(1-\alpha)$ percentile of a standard normal distribution. We refer to this approach, where we calculate the BMDL using the delta method and Wald test statistic (and which relies upon the asymptotic normal distribution of the Wald 
statistic) as BMDLN.

\subsubsection{Likelihood Ratio Based Confidence Interval}

We also consider a second approach to constructing a confidence interval for the BMD which is based on inverting the Likelihood Ratio Test (LRT) of Neyman and Pearson (1928) and Wilks (1938). The likelihood, and by extension the log-likelihood, is parameterized by $\boldsymbol{\theta}$ and is not explicitly parameterized by the BMD. However, it is indirectly a function of the BMD and we can use the log-likelihood to perform inference on the BMD.

To parameterize the log-likelihood as a function of a specified value for the BMD, $d$, we make use of $\Theta_{d}=\{\boldsymbol{\theta} \mid h(\boldsymbol{\theta})=d\}$, the subspace of the full parameter space which corresponds to a BMD of $d$. If all other parameters are nuisance parameters then we are interested in the maximum obtainable value of the log-likelihood over this space. We thus obtain the function

$$
\tilde{l}(d)=\max _{\boldsymbol{\theta} \in \Theta_{d}} l(\boldsymbol{\theta})
$$

which is referred to as the profile log-likelihood. Note that

$$
\max _{d} \tilde{l}(d)=\max _{d} \max _{\boldsymbol{\theta} \in \Theta_{d}} l(\boldsymbol{\theta})=\max _{\boldsymbol{\theta}} l(\boldsymbol{\theta})
$$

and so if $\hat{d}=h(\hat{\boldsymbol{\theta}})$ is the MLE of the BMD then $\tilde{l}(\hat{d})=\tilde{l}(h(\hat{\boldsymbol{\theta}}))=l(\hat{\boldsymbol{\theta}})$.

For some ordinal models we can reparameterize $\boldsymbol{\theta}$ directly, in particular those models for which the cumulative probabilities of each category are monotone increasing with respect to dose. Monotonicity is a desirable and natural characteristic for 
dose-response data and many ordinal models, such as those defined by (1.4) and (2.21), possess this property.

From the LRT we have that $2[\tilde{l}(\hat{d})-\tilde{l}(d)] \sim \chi_{1}^{2}$ where $\chi_{1}^{2}$ is a chi-square distribution with one degree of freedom. This implies that

$$
\left\{d \mid 2[\tilde{l}(\hat{d})-\tilde{l}(d)] \leq \chi_{1,1-\alpha}^{2}\right\}
$$

is a $(1-\alpha)$ confidence set for $d$. A two-sided confidence interval for $h(\boldsymbol{\theta})$ with at least a $(1-\alpha)$ confidence level can be formed by taking the minimum and maximum values of $d$ in this set. Combined, the lower and upper tail probabilities are no more than $\alpha$, and if we assume that the tail probabilities are equal we can then form a one-sided confidence interval. (This assumption holds asymptotically, see Equation (2.19).)

For a one-sided confidence interval with a $(1-\alpha)$ confidence level we take the lower bound of a two-sided interval constructed with a $(1-2 \alpha)$ confidence level. Thus

$$
b_{l}(\hat{d}, \alpha)=\left\{\min _{d} h(d) \mid 2[\tilde{l}(\hat{d})-\tilde{l}(d)] \leq \chi_{1-2 \alpha}^{2} \cdot\right\}
$$

is the BMDL at level $\alpha$ based on likelihood ratio test. We refer to this approach as BMDLX.

\section{Comparison of BMDLN and BMDLX}

Unfortunately, the BMDLN method can result in values that fall outside of the dosage range, namely, which are are negative. Due to its closed form, it is simpler to compute than the BMDLX, but is less powerful. In general the BMDLX does not have a closed form, and since it is calculated numerically it is more computationally intensive. 


\subsection{Simulation}

In this section, we evaluate via a simulation study the properties of the expression for the OBMD proposed in Section 3.3. Recall that the OBMD is defined in this study by using (3.12) for the probability of an adverse response in conjunction with the equation for additional risk given in (3.1).

\subsubsection{Investigation of the OBMD Estimator}

We begin by assuming that the true underlying data generation mechanism can be described by a cumulative link model with shared effects (CUR) as provided in (1.5). In particular, we initially consider an ordinal outcome with $C=5$ categories, and set the parameter values for the CUR model as $\boldsymbol{\theta}=(1.5,2,3,4.5,-0.1)^{\top}$, so that $\alpha_{1}=1.5, \alpha_{2}=2, \alpha_{3}=3, \alpha_{4}=4.5$, and $\beta=-0.1$. Provided that a value of the risk $R$ is specified in (3.1), it is possible to compute the true value of the ordinal benchmark dose by using this equation in tandem with (3.12). Setting $R=0.10$ yields a true value of OBMD of 6.104 in this case, which does not change with different numbers of dose levels, or trials per dose.

We proceeded to simulate data from this model; we began by considering a situation that would emulate an experiment with five dose levels, and twenty trials per dose level, thereby yielding a data set of 100 observations. For this data set, we computed an estimate of the OBMD. We continued to generate a total of 1,000 such samples of 100 observations each. For each we determined an estimate of the OBMD; we also calculated the sample standard deviation of the OBMD estimates over the 1,000 simulated data sets. The mean and standard deviation of the OBMD estimates are presented in the row corresponding to $C=5$ and design (i) in Panel A of Table 3.1. 


\begin{tabular}{ccccccc}
\hline \multirow{2}{*}{ Panel A: BMD for nested designs } & & & \\
Design & C & $\begin{array}{c}\text { Number } \\
\text { of doses }\end{array}$ & $\begin{array}{c}\text { Repetitions } \\
\text { per dose }\end{array}$ & \multicolumn{3}{c}{ BMD } \\
\cline { 5 - 7 } & & & & True & Mean & SE \\
\hline i & 3 & 5 & 20 & 5.999 & 6.440 & 1.726 \\
ii & 3 & 10 & 35 & 5.999 & 6.155 & 0.772 \\
iii & 3 & 20 & 50 & 5.999 & 6.045 & 0.426 \\
\hline i & 4 & 5 & 20 & 6.027 & 6.474 & 1.769 \\
ii & 4 & 10 & 35 & 6.027 & 6.183 & 0.781 \\
iii & 4 & 20 & 50 & 6.027 & 6.073 & 0.433 \\
\hline i & 5 & 5 & 20 & 6.104 & 6.566 & 1.837 \\
ii & 5 & 10 & 35 & 6.104 & 6.262 & 0.778 \\
iii & 5 & 20 & 50 & 6.104 & 6.149 & 0.431 \\
\hline
\end{tabular}

Panel B: BMD for designs with $C=4$

\begin{tabular}{ccccccc}
\hline \multirow{2}{*}{ Design } & C & \multirow{2}{*}{$\begin{array}{c}\text { Number } \\
\text { of doses }\end{array}$} & $\begin{array}{c}\text { Repetitions } \\
\text { per dose }\end{array}$ & \multicolumn{3}{c}{ BMD } \\
\cline { 5 - 7 } & & & & True & Mean & SE \\
\hline i & 4 & 5 & 20 & 6.027 & 6.474 & 1.769 \\
- & 4 & 5 & 35 & 6.027 & 6.343 & 1.096 \\
- & 4 & 5 & 50 & 6.027 & 6.166 & 0.851 \\
\hline- & 4 & 10 & 20 & 6.027 & 6.359 & 1.219 \\
- & 4 & 10 & 35 & 6.027 & 6.183 & 0.781 \\
ii & 4 & 10 & 50 & 6.027 & 6.116 & 0.615 \\
\hline- & 4 & 20 & 20 & 6.027 & 6.171 & 0.738 \\
\hline- & 4 & 20 & 35 & 6.027 & 6.110 & 0.535 \\
- & 4 & 20 & 50 & 6.027 & 6.073 & 0.433 \\
\hline iii & 4 & & & & &
\end{tabular}

Table 3.1: A selection of simulation results investigating OBMD; results across different values of $C$ for three nested designs appear in Panel A, and results for $C=4$ across designs with various number of doses and repetitions per dose appear in Panel B. 
Next, we repeated the entire simulation above for different combinations of dose levels and number of trials per dose. Specifically, we generated 1,000 simulated data sets for each of sixteen cases that were defined by combining each of $5,10,20$, and 50 dose levels with 20, 35, 50, and 100 observations per dose level. Thus, the largest simulated data sets would have consisted of 5,000 observations. The last three rows of Panel A in Table 3.1 present the mean and standard deviation of the OBMD estimates obtained for three of the sixteen cases considered; the complete set of results can be found in Appendix B. It is worthy to note that the estimator of OBMD seems biased; however this bias becomes smaller as the number of dose levels and observations per dose increase. In addition, the variability in the estimator is notably smaller with relatively more dose levels and observations.

We also wish for an estimator of benchmark dose that is robust to the number of categories chosen for the ordinal outcome variable in an experiment. For this reason, we investigated the performance of the proposed estimator by repeating the entire simulation of sixteen cases described above for two additional cases distinguished by the number of categories for the response. One used $C=4$ categories that were chosen by combining the second and third levels of the ordinal outcome variable in the five-category case and setting $\boldsymbol{\theta}=(1.5,3,4.5,-0.1)^{\top}$, the other used $C=3$ categories, created by merging the last two categories in the four-outcome case, and setting $\boldsymbol{\theta}=(1.5,3,-0.1)^{\top}$. Note that the construction of the true $\boldsymbol{\theta}$ for $C=3,4,5$ are such that the $C=3$ case is nested within $C=4$ which is nested within $C=5$. As such, we do no generate new data sets but rather use the same 1,000 data sets as with the $C=5$ case, and simply merge the appropriate categories. For $R=0.10$, the true OBMD values were 5.999 and 6.027 for the three- and four-category cases, respectively. Thus, the true OBMD remained approximately constant with changes 
to the manner in which the ordinal response was defined.

As stated above, selected results for the five-category case are presented in the last three rows of Panel A in Table 3.1; by contrast, the previous rows present analogous results for the cases where the ordinal outcome is reduced to three and four categories, respectively. The results in Panel A in Table 3.1 demonstrate that the proposed measure for ordinal benchmark dose is robust to the number of categories specified for the ordinal outcome. Similar to the case for $C=5$, a complete set of results for

all sixteen combinations of dose levels and trials per dose for each of $C=3$ and $C=4$ is presented in Appendix B.

When the results for the five-category case in Table 3.1 were discussed, it was mentioned that the estimator of OBMD is biased; however as the number of dose levels and trials per dose increase, both the bias and variability in the estimator decrease. This is also true for $C=3$ and $C=4$. As a further illustration, Panel B in Table 3.1 presents more of the results associated with the sixteen combinations of dose level and trials per dose for the model with $C=4$. Specifically, nine of the sixteen combinations are shown. These results illustrate the reduction in the bias and variability of the OBMD estimator as the number of dose levels and trials per dose increase. In fact, for the case of twenty dose levels and fifty repetitions per dose, the bias is negligible. Note that here we only investigated the OBMD for $C=3,4,5$, however its definition readily allows for a larger number of categories.

\subsubsection{Investigation of the Lower Confidence Limit of OBMD}

In addition to the above, we also investigated the performance of the two methods proposed in Section 3.4 for estimating the lower confidence limit of the benchmark dose, BMDL. The first of these methods, BMDLN, is based on the delta method and 
uses the standard normal distribution to define this limit. Under the other method, BMDLX, a likelihood ratio confidence interval based on the chi-square distribution with one degree of freedom is determined.

Recall that for each of the models above with $C=3,4$, and 5 categories, we have investigated sixteen different cases defined by each of four total dose levels of 5,10 , 20, and 50 being paired with 20, 35, 50, and 100 trials per dose. For each of these forty-eight cases, we initially computed the true values of BMDLN and BMDLX. To calculate the true BMDLN, we evaluate Fisher's information matrix, $\mathcal{J}(\boldsymbol{\theta})$, at the true value $\boldsymbol{\theta}$ and the expected value of the data, $\left\{\boldsymbol{\pi}_{1}(\boldsymbol{\theta}), \ldots, \boldsymbol{\pi}_{n}(\boldsymbol{\theta})\right\}$, and take the inverse of this matrix to be the asymptotic variance of $\hat{\boldsymbol{\theta}}$. This variance matrix is subsequently used in calculating $\widehat{V}$ in (3.13). Similarly, for BMDLX, we evaluate the two profile log-likelihoods, $\tilde{l}(d)$ and $\tilde{l}(\hat{d})$, in (3.15) at the expected value of the data and of parameters, $\mathrm{E}(\hat{\boldsymbol{\theta}})=\boldsymbol{\theta}$ and $\mathrm{E}(\hat{d})=d$. Note that $d 0$ is the true BMD and the solution to (3.1) under $\boldsymbol{\theta}, R=p_{\boldsymbol{\theta}}(d)-p_{\boldsymbol{\theta}}(0)$.

In what follows, we set the level of significance to 0.05 , thereby allowing for the appropriate standard normal and chi-square percentiles to be obtained. For each of the forty-eight combinations considered here that are distinguished by the number of categories, number of dose levels, and number of trials per dose, there is a total of 1,000 simulated data sets available. For each data set associated with a particular combination, setting $R=0.10$ and the level of significance to 0.05 , we determined estimates of BMDLN and BMDLX, and verified whether or not the appropriate true value was greater than or equal to the analogous simulation estimate. For a given combination of categories, dose levels, and trials, we also determined, for each of BMDLN and BMDLX, the mean and standard deviations of the estimates, along with the proportion of times that the true value was at least as large as the estimate, 
taking the latter as a coverage estimate for an expected $95 \%$ nominal rate.

In Appendix B we present the results for all the forty-eight combinations which are considered, a selection is shown in Tables 3.2 and 3.3. In addition to allowing for a comparison of the performance of the two approaches, a contrast of these two tables facilitates the assessment of altering the number of categories for the ordinal outcome. From Table 3.2, it can be seen that for a fixed number of dose levels, and trials per dose, the true values of BMDLN and BMDLX are quite robust with respect to the number of categories specified for the ordinal outcome. For example, for 20 dose levels and 50 repetitions per dose, the true BMDLN values are 5.324, 5.342 , and 5.422 for ordinal outcome variables consisting of three, four, and five categories respectively. Analogous results for BMDLX are 5.432, 5.453, and 5.532, respectively. Not surprisingly, for a fixed level of categories, the true values of BMDLN and BMDLX increase as the number of total trials increase. For example, for the case of five categories, the true value of BMDLX increases from 4.782 for 100 total trials ( 5 dose levels, 20 repetitions) to 5.532 for 1,000 total trials (20 dose levels, 50 repetitions). In essence, it could be argued intuitively here that the tolerable lower limit of the benchmark dose can be relaxed and increased somewhat when there is more information contained in the sample.

Tables 3.2 and 3.3 also demonstrate that the estimator for BMDLN is significantly biased when the number of dose levels and total trials is relatively small. While the bias becomes quite negligible for a large number of total trials, the lower bound confidence limit for the OBMD is not able to obtain the appropriate coverage; in fact, it over-covers. Thus, despite the unsurprising fact that the standard error of the BMDLN decreases as the total number of trials increases for a fixed number of categories for the ordinal outcome, the estimate used to approximate the variability in 


\begin{tabular}{|c|c|c|c|c|c|c|c|}
\hline \multicolumn{8}{|c|}{ Panel A: BMDLN for nested designs } \\
\hline \multirow{2}{*}{ Design } & \multirow{2}{*}{$\mathrm{C}$} & \multirow{2}{*}{$\begin{array}{l}\text { Number } \\
\text { of doses }\end{array}$} & \multirow{2}{*}{$\begin{array}{l}\text { Repetitions } \\
\text { per dose }\end{array}$} & \multicolumn{4}{|c|}{ BMDLN } \\
\hline & & & & True & Mean & $\mathrm{SE}$ & Coverage \\
\hline $\mathrm{i}$ & 3 & 5 & 20 & 4.110 & 3.758 & 1.668 & 0.999 \\
\hline ii & 3 & 10 & 35 & 4.904 & 4.926 & 0.308 & 1.000 \\
\hline iii & 3 & 20 & 50 & 5.324 & 5.345 & 0.260 & 0.985 \\
\hline $\mathrm{i}$ & 4 & 5 & 20 & 4.110 & 3.711 & 2.218 & 0.999 \\
\hline ii & 4 & 10 & 35 & 4.915 & 4.938 & 0.309 & 1.000 \\
\hline iii & 4 & 20 & 50 & 5.342 & 5.363 & 0.262 & 0.984 \\
\hline $\mathrm{i}$ & 5 & 5 & 20 & 4.201 & 3.792 & 2.121 & 1.000 \\
\hline ii & 5 & 10 & 35 & 4.998 & 5.024 & 0.303 & 1.000 \\
\hline iii & 5 & 20 & 50 & 5.422 & 5.443 & 0.262 & 0.984 \\
\hline
\end{tabular}

Panel B: BMDLX for nested designs

\begin{tabular}{|c|c|c|c|c|c|c|c|}
\hline \multirow{2}{*}{ Design } & \multirow{2}{*}{$\mathrm{C}$} & \multirow{2}{*}{$\begin{array}{l}\text { Number } \\
\text { of doses }\end{array}$} & \multirow{2}{*}{$\begin{array}{l}\text { Repetitions } \\
\text { per dose }\end{array}$} & \multicolumn{4}{|c|}{ BMDLX } \\
\hline & & & & True & Mean & SE & Coverage \\
\hline i & 3 & 5 & 20 & 4.677 & 4.787 & 0.813 & 0.958 \\
\hline ii & 3 & 10 & 35 & 5.150 & 5.223 & 0.453 & 0.936 \\
\hline iii & 3 & 20 & 50 & 5.432 & 5.460 & 0.299 & 0.948 \\
\hline $\mathrm{i}$ & 4 & 5 & 20 & 4.691 & 4.800 & 0.818 & 0.954 \\
\hline ii & 4 & 10 & 35 & 5.168 & 5.241 & 0.456 & 0.938 \\
\hline iii & 4 & 20 & 50 & 5.453 & 5.480 & 0.303 & 0.949 \\
\hline $\mathrm{i}$ & 5 & 5 & 20 & 4.782 & 4.884 & 0.666 & 0.950 \\
\hline ii & 5 & 10 & 35 & 5.251 & 5.324 & 0.452 & 0.935 \\
\hline iii & 5 & 20 & 50 & 5.532 & 5.559 & 0.302 & 0.944 \\
\hline
\end{tabular}

Table 3.2: A selection of simulation results investigating estimators of the lower confidence limit of OBMD across different values of $C$ for three nested designs. The results for a nominal confidence level of 95\% for the BMDLN and BMDLX estimators appear in Panels A and $\mathrm{B}$ respectively. 


\begin{tabular}{|c|c|c|c|c|c|c|c|}
\hline \multirow{2}{*}{ Design } & \multirow{2}{*}{$\mathrm{C}$} & \multirow{2}{*}{$\begin{array}{l}\text { Number } \\
\text { of doses }\end{array}$} & \multirow{2}{*}{$\begin{array}{l}\text { Repetitions } \\
\text { per dose }\end{array}$} & \multicolumn{4}{|c|}{ BMDLN } \\
\hline & & & & True & Mean & $\mathrm{SE}$ & Coverage \\
\hline $\mathrm{i}$ & 4 & 5 & 20 & 4.110 & 3.711 & 2.218 & 0.999 \\
\hline - & 4 & 5 & 35 & 4.578 & 4.547 & 0.393 & 0.999 \\
\hline - & 4 & 5 & 50 & 4.814 & 4.798 & 0.317 & 1.000 \\
\hline - & 4 & 10 & 20 & 4.556 & 4.487 & 0.502 & 1.000 \\
\hline ii & 4 & 10 & 35 & 4.915 & 4.938 & 0.309 & 1.000 \\
\hline - & 4 & 10 & 50 & 5.097 & 5.115 & 0.299 & 0.998 \\
\hline - & 4 & 20 & 20 & 4.943 & 4.965 & 0.284 & 1.000 \\
\hline - & 4 & 20 & 35 & 5.208 & 5.238 & 0.278 & 0.991 \\
\hline iii & 4 & 20 & 50 & 5.342 & 5.363 & 0.262 & 0.984 \\
\hline
\end{tabular}

Panel B: BMDLX for designs with $C=4$

\begin{tabular}{cccccccc}
\hline \multirow{2}{*}{ Design } & C & \multirow{2}{*}{$\begin{array}{c}\text { Number } \\
\text { of doses }\end{array}$} & $\begin{array}{c}\text { Repetitions } \\
\text { per dose }\end{array}$ & \multicolumn{4}{c}{ BMDLX } \\
\cline { 6 - 8 } & & & & True & Mean & SE & Coverage \\
\hline i & 4 & 5 & 20 & 4.691 & 4.800 & 0.818 & 0.954 \\
- & 4 & 5 & 35 & 4.948 & 5.076 & 0.554 & 0.945 \\
- & 4 & 5 & 50 & 5.090 & 5.136 & 0.480 & 0.958 \\
\hline - & 4 & 10 & 20 & 4.959 & 5.082 & 0.551 & 0.944 \\
ii & 4 & 10 & 35 & 5.168 & 5.241 & 0.456 & 0.938 \\
- & 4 & 10 & 50 & 5.283 & 5.324 & 0.391 & 0.953 \\
\hline- & 4 & 20 & 20 & 5.191 & 5.259 & 0.427 & 0.945 \\
- & 4 & 20 & 35 & 5.360 & 5.406 & 0.348 & 0.949 \\
iii & 4 & 20 & 50 & 5.453 & 5.480 & 0.303 & 0.949 \\
\hline
\end{tabular}

Table 3.3: A selection of simulation results investigating estimators of the lower confidence limit of OBMD for $C=4$ across designs with various number of doses and repetitions per dose. The results for a nominal confidence level of $95 \%$ for the BMDLN and BMDLX estimators appear in Panels A and B respectively. 
BMDLN is too large. By contrast, the results obtained using BMDLX are extremely encouraging. The estimator has little bias across all combinations presented, and the lower confidence bound for the OBMD achieves the appropriate coverage in all cases.

\subsection{Conclusion}

In this chapter, attention was focused on the development of a reference dose measure for ordinal outcome data. Specifically, we propose an alternative method for defining the benchmark dose, BMD, for ordinal outcome data. The approach yields an estimator that is robust to the number of ordinal categories into which we divide the response. In addition, the estimator is consistent with currently accepted definitions of the BMD for quantal and continuous data when the number of categories for the ordinal response is two, or becomes extremely large, respectively. We also suggested two methods for determining an interval reflecting the lower confidence limit of the BMD; one based on the delta method, the other on a likelihood ratio approach. We showed via a simulation study that intervals based on the latter approach are able to achieve the nominal level of coverage. 


\section{Bibliography}

Agresti, A. (2010). Analysis of Ordinal Categorical Data, volume 14. Wiley, Hoboken, NJ, USA, 2nd edition.

Allen, B. C., Kavlock, R. J., Kimmel, C. A., and Faustman, E. M. (1994). DoseâĂŤresponse assessment for developmental toxicity III. statistical models. Toxicological Sciences, 23(4):496-509.

Ananth, C. V. and Kleinbaum, D. G. (1997). Regression models for ordinal responses: a review of methods and applications. International journal of epidemiology, 26(6):1323-1333.

Barnes, D. G., Daston, G. P., Evans, J. S., Jarabek, A. M., Kavlock, R. J., Kimmel, C. A., Park, C., and Spitzer, H. L. (1995). Benchmark dose workshop: criteria for use of a benchmark dose to estimate a reference dose. Regulatory Toxicology and Pharmacology, 21(2):296-306.

Bishop, Y. M., Fienberg, S. E., and Holland, P. W. (1975). Discrete multivariate analysis: theory and practice. Cambridge.

Chen, C.-C. and Chen, J. J. (2014). Benchmark dose calculation for ordered categorical responses. Risk Analysis, 34(8):1435-1447. 
Chuang-Stein, C. and Agresti, A. (1997). Tutorial in biostatistics a review of tests for detecting a monotone dose-response relationship with ordinal response data. Statistics in Medicine, 16(22):2599-2618.

Crump, K. S. (1984). A new method for determining allowable daily intakes. Fundamental and Applied Toxicology, 4(5):854-871.

Crump, K. S. (1995). Calculation of benchmark doses from continuous data. Risk Analysis, 15(1):79-89.

Crump, K. S. (2002). Critical issues in benchmark calculations from continuous data. Critical Reviews in Toxicology, 32(3):133-153.

de Boor, C. (1978). A practical guide to splines. Mathematics of Computation.

EFSA (2009). Guidance of the scientific committee on a request from EFSA on the use of the benchmark dose approach in risk assessment. EFSA Journal, 1(1150):1-72.

Eilers, P. H. C. and Marx, B. D. (1996). Flexible smoothing with b -splines and penalties. Statistical Science, 11(2):89-121.

Faes, C., Geys, H., Aerts, M., Molenberghs, G., and Catalano, P. J. (2004). Modeling combined continuous and ordinal outcomes in a clustered setting. Journal of agricultural, biological, and environmental statistics, 9(4):515-530.

Gaylor, D., Ryan, L., Krewski, D., and Zhu, Y. (1998). Procedures for calculating benchmark doses for health risk assessment. Regulatory Toxicology and Pharmacology, 28(2):150-164.

Gaylor, D. W. (1983). The use of safety factors for controlling risk. Journal of Toxicology and Environmental Health, Part A Current Issues, 11(3):329-336. 
Gaylor, D. W. (1996). Quantalization of continuous data for benchmark dose estimation. Regulatory toxicology and pharmacology, 24(3):246-250.

Gaylor, D. W. and Slikker, W. (1990). Risk assessment for neurotoxic effects. Neurotoxicology, 11(2):211-218.

Hastie, T. and Tibshirani, R. (1987). Non-parametric logistic and proportional odds regression. Applied Statistics, 36(3):260-276.

Hastie, T. J. and Tibshirani, R. J. (1990). Generalized additive models, volume 43. CRC Press.

Hill, A. V. (1910). The possible effects of the aggregation of the molecules of haemoglobin on its dissociation curves. Journal of Physiology, 40:4-7.

Jarrett, R. G., Morgan, B. J. T., and Liow, S. (1981). The effects of viruses on death and deformity rates in chicken eggs, consulting report VT 81/37. Technical report, CSIRO Division of Mathematics and statistics, Melbourne.

Kelly, C. and Rice, J. (1990). Monotone smoothing with application to dose-response curves and the assessment of synergism. Biometrics, 46(4):1071-1085.

Kimmel, C. A. and Gaylor, D. W. (1988). Issues in qualitative and quantitative risk analysis for developmental toxicology1. Risk analysis, 8(1):15-20.

Kodell, R. L. and West, R. W. (1993). Upper confidence limits on excess risk for quantitative responses. Risk Analysis, 13(2):177-182.

Kong, M. and Eubank, R. L. (2006). Monotone smoothing with application to dose-response curve. Communications in Statistics - Simulation and Computation, 35(4):991-1004. 
Kooperberg, C., Bose, S., and Stone, C. J. (1997). Polychotomous regression. Journal of the American Statistical Association, 92(437):117-127.

Kremer, J. M., Cohen, S., Wilkinson, B. E., Connell, C. A., French, J. L., GomezReino, J., Gruben, D., Kanik, K. S., Krishnaswami, S., Pascual-Ramos, V., Wallenstein, G., and Zwillich, S. H. (2012). A phase IIb dose-ranging study of the oral jak inhibitor tofacitinib (cp-690,550) versus placebo in combination with background methotrexate in patients with active rheumatoid arthritis and an inadequate response to methotrexate alone. Arthritis \& Rheumatism, 64:970-981.

Krewski, D. and Zhu, Y. (1994). Applications of multinomial dose-response models in developmental toxicity risk assessment. Risk Analysis, 14(4):613-627.

Leisenring, W. and Ryan, L. (1992). Statistical properties of the NOAEL. Regulatory Toxicology and Pharmacology, 15:161-171.

Lu, M., Zhang, Y., and Huang, J. (2009). Semiparametric estimation methods for panel count data using monotone b-splines. Journal of the American Statistical Association, 104(487):1060-1070.

Lu, Y. and Chiang, A. Y. (2011). Combining biomarker identification and doseresponse modeling in early clinical development. Statistics in Biopharmaceutical

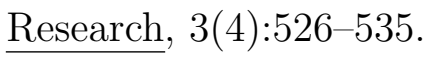

Mbah, A. K., Hamisu, I., Naik, E., and Salihu, H. M. (2014). Estimating benchmark exposure for air particulate matter using latent class models. Risk Analysis, 34(11):2053-2062. 
McCullagh, P. (1980). Regression models for ordinal data. Journal of the royal statistical society. Series B (Methodological), 42(2):109-142.

Moerbeek, M., Piersma, A. H., and Slob, W. (2004). A comparison of three methods for calculating confidence intervals for the benchmark dose. Risk analysis, 24(1):3140.

Morgan, B. (1992). Analysis of Quatal Response Data. Monographs on Statistics and Applied Probability. Chapman \& Hall.

Murrell, J. A., Portier, C. J., and Morris, R. W. (1998). Characterizing dose-response i: Critical assessment of the benchmark dose concept. Risk Analysis, 18(1):13-26.

Nelder, J. A. and Wedderburn, R. W. M. (1972). Generalized linear models. Journal of the Royal Statistical Society, Series A, General, 135:370-384.

Neyman, J. and Pearson, E. S. (1928). On the use and interpretation of certain test criteria for purposes of statistical inference: Part i. Biometrika, pages 175-240.

Pratt, J. W. (1981). Concavity of the log likelihood. Journal of the American Statistical Association, 76(373):103-106.

R Core Team (2015). R: A Language and Environment for Statistical Computing. R Foundation for Statistical Computing, Vienna, Austria.

Ramsay, J. and Silverman, B. W. (2005). Functional Data Analysis. Springer-Verlag New York, second edition.

Ramsay, J. O. (1988). Monotone regression splines in action. Statistical Science, $3(4): 425-441$. 
Regan, M. M. and Catalano, P. J. (2000). Regression models and risk estimation for mixed discrete and continuous outcomes in developmental toxicology. Risk Analysis, 20(3):363-376.

Regan, M. M. and Catalano, P. J. (2002). Regression models and risk estimation for mixed discrete and continuous outcomes in developmental toxicology. Risk Analysis, 20(3):363-376.

RIVM (2012). PROAST: Software for doseâĂŞresponse modeling and benchmark dose analysis. RIVM.

Ruppert, D. (2002). Selecting the number of knots for penalized splines. Journal of Computational and Graphical Statistics, 11(4):735-757.

Sand, S., Victorin, K., and Filipsson, A. F. (2008). The current state of knowledge on the use of the benchmark dose concept in risk assessment. Journal of Applied Toxicology, 28(4):405-421.

Sand, S., von Rosen, D., Victorin, K., and Falk Filipsson, A. (2006). Identification of a critical dose level for risk assessment: Developments in benchmark dose analysis of continuous endpoints. Toxicological Sciences, 90(1):241-251.

Slob, W. (2002). Dose-response modeling of continuous endpoints. Toxicological Sciences, 66(2):298-312.

Slob, W. and Pieters, M. (1998). A probabilistic approach for deriving acceptable human intake limits and human health risks from toxicological studies: general framework. Risk Analysis, 18(6):787-798. 
Tutz, G. (2011). Regression for Categorical Data. Number 34 in Cambridge Series in Statistical and Probabilistic Mathematics. Cambridge University Press.

USEPA (1995). The use of the benchmark dose approach in health risk assessment. Risk assessment forum, Environmental Protection Agency.

USEPA (2010). Toxicological review of 1,1,2,2-tetrachloroethane (CAS no. 79-34-5). Technical report, Environmental Protection Agency.

USEPA (2012). Benchmark dose technical guidance. Washington, DC.

Wilks, S. S. (1938). The large-sample distribution of the likelihood ratio for testing composite hypotheses. The Annals of Mathematical Statistics, 9(1):60-62.

Xie, M. and Simpson, D. G. (1999). Regression modeling of ordinal data with nonzero baselines. Biometrics, 55(1):308-316.

Yee, T. W. (2015). Vector Generalized Linear and Additive Models: with an Implementation in R. Springer-Verlag New York.

Yee, T. W. and Wild, C. J. (1996). Vector generalized additive models. Journal of the Royal Statistical Society: Series B (Statistical Methodology), 58(3):481-493. 
Appendix A

Simulation Results for Spline Models 







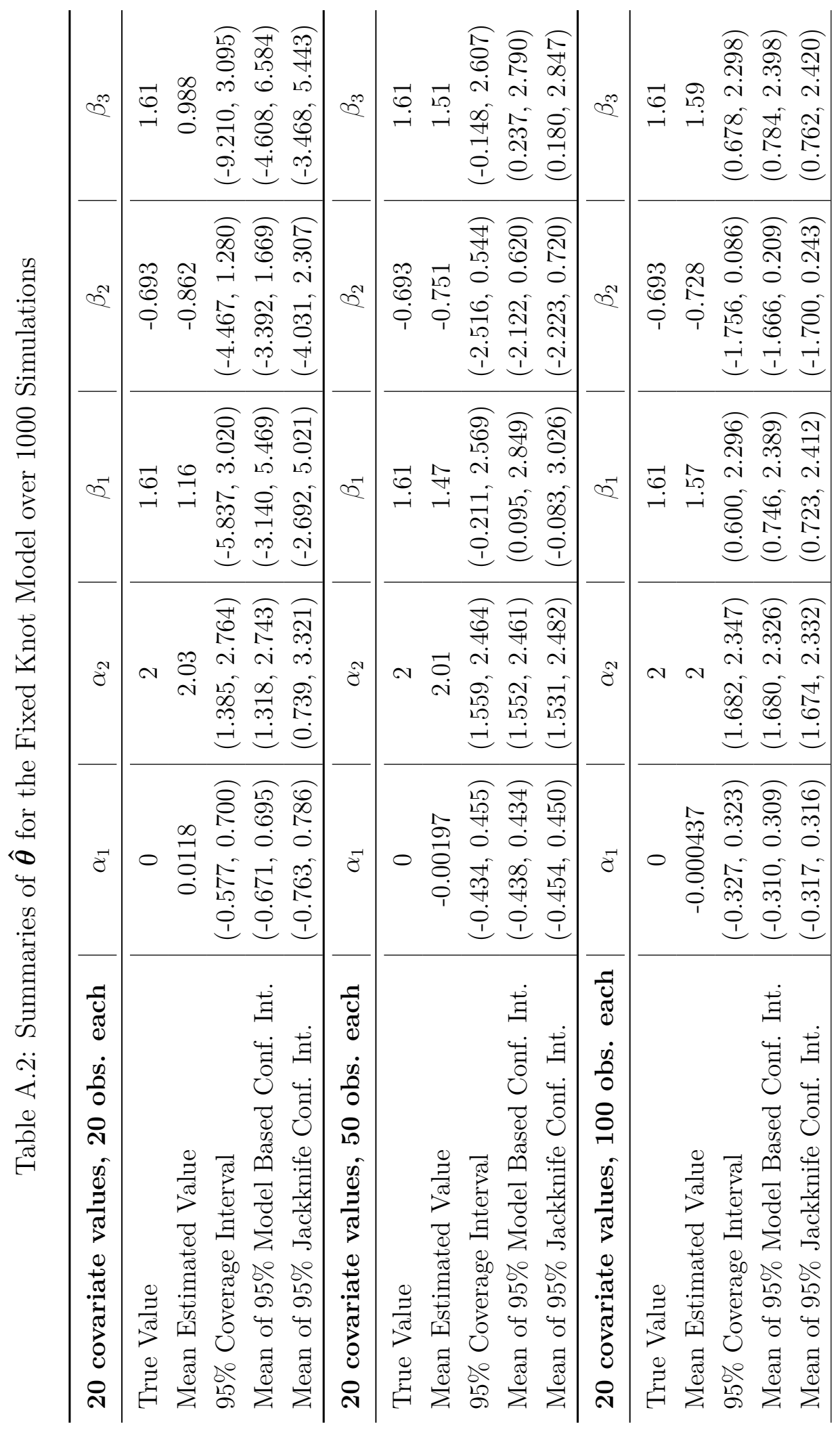




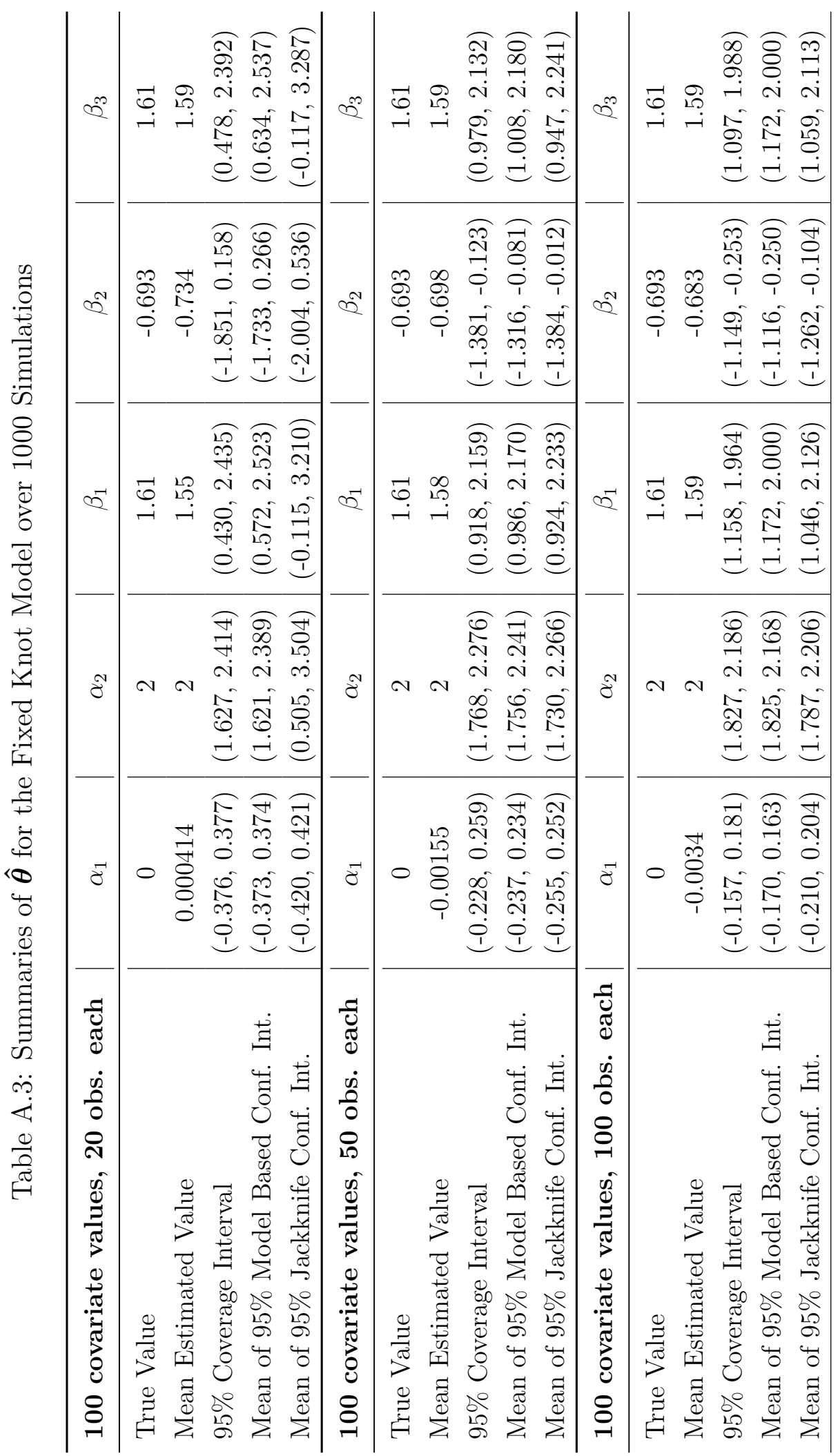




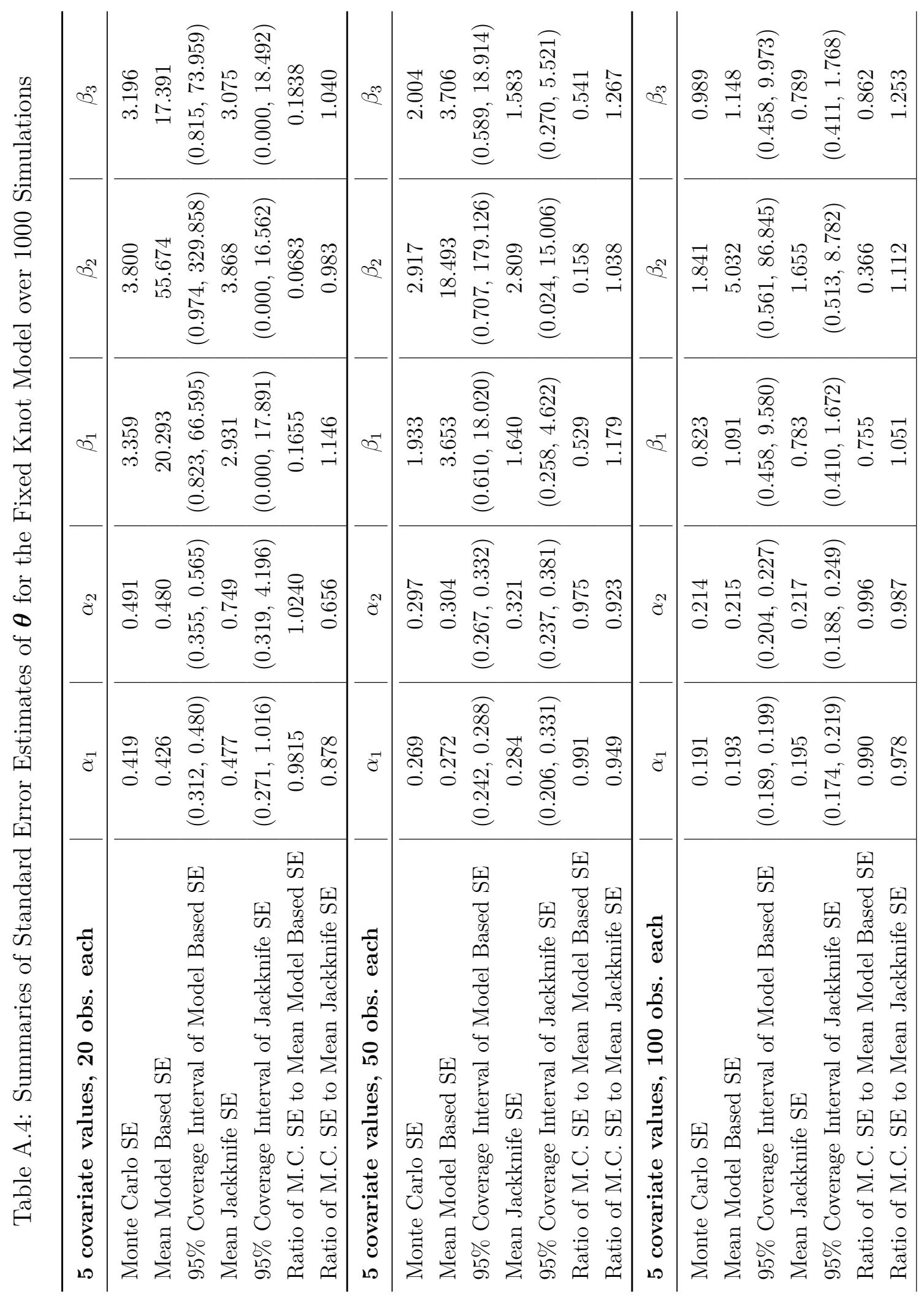




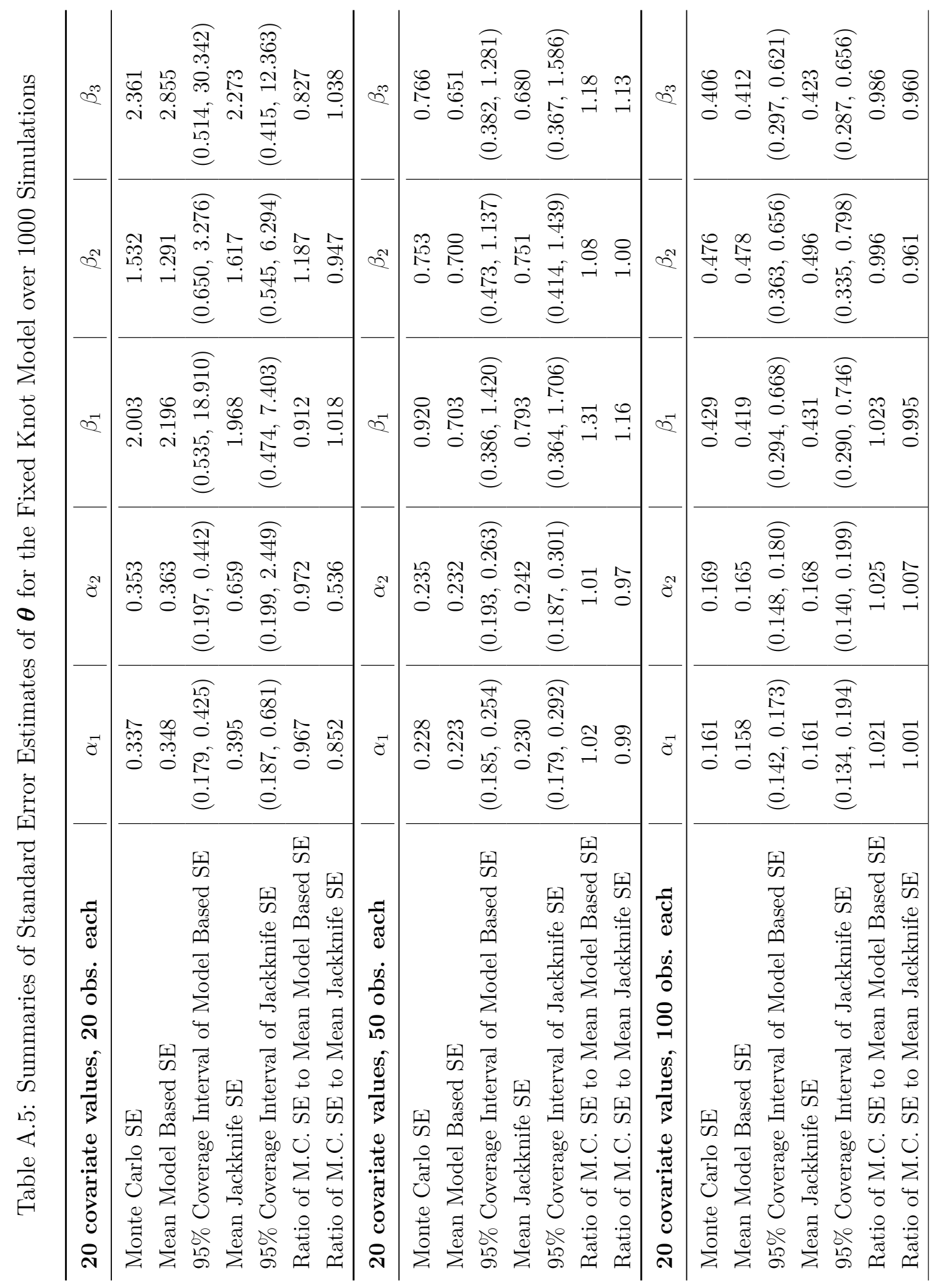









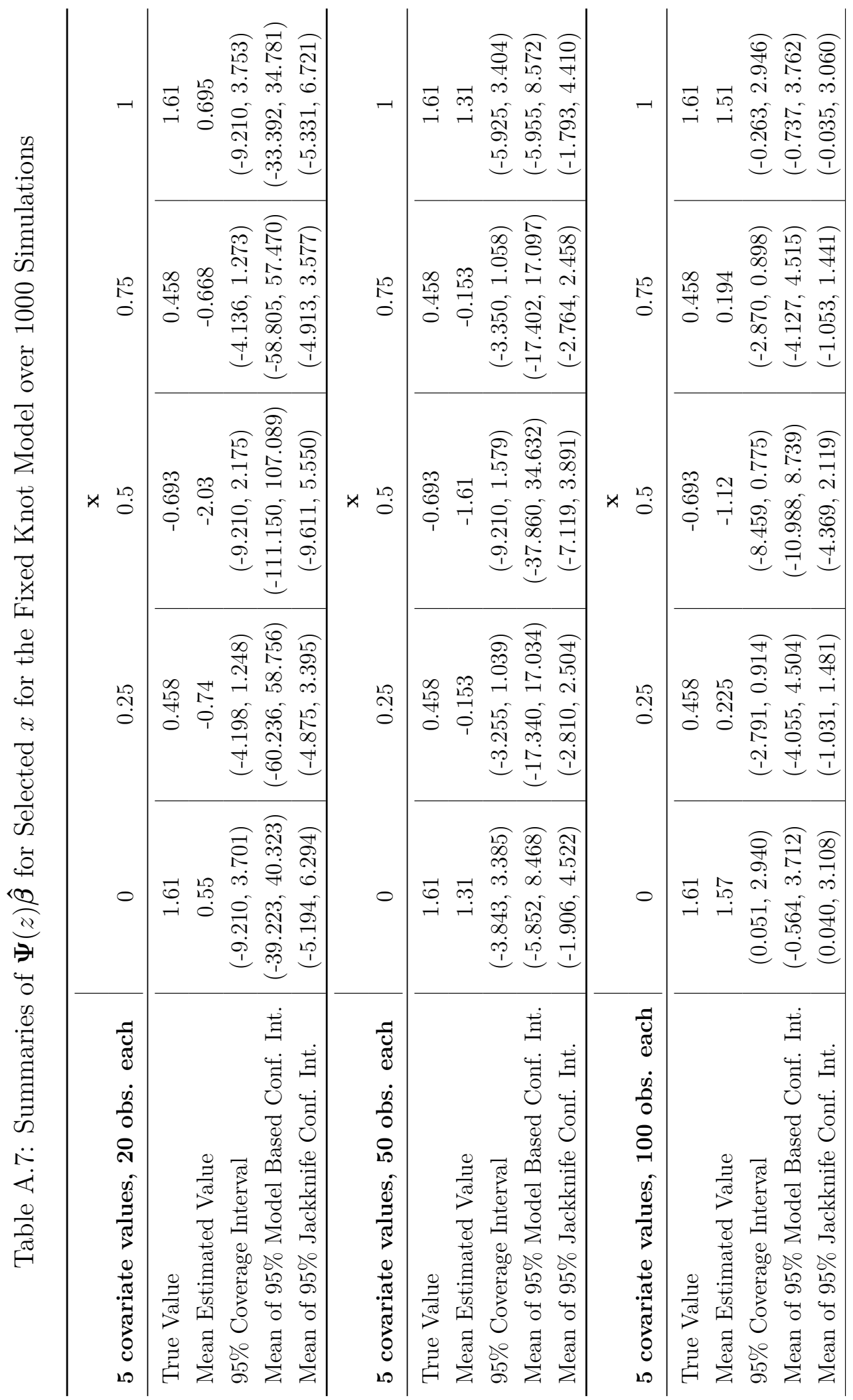




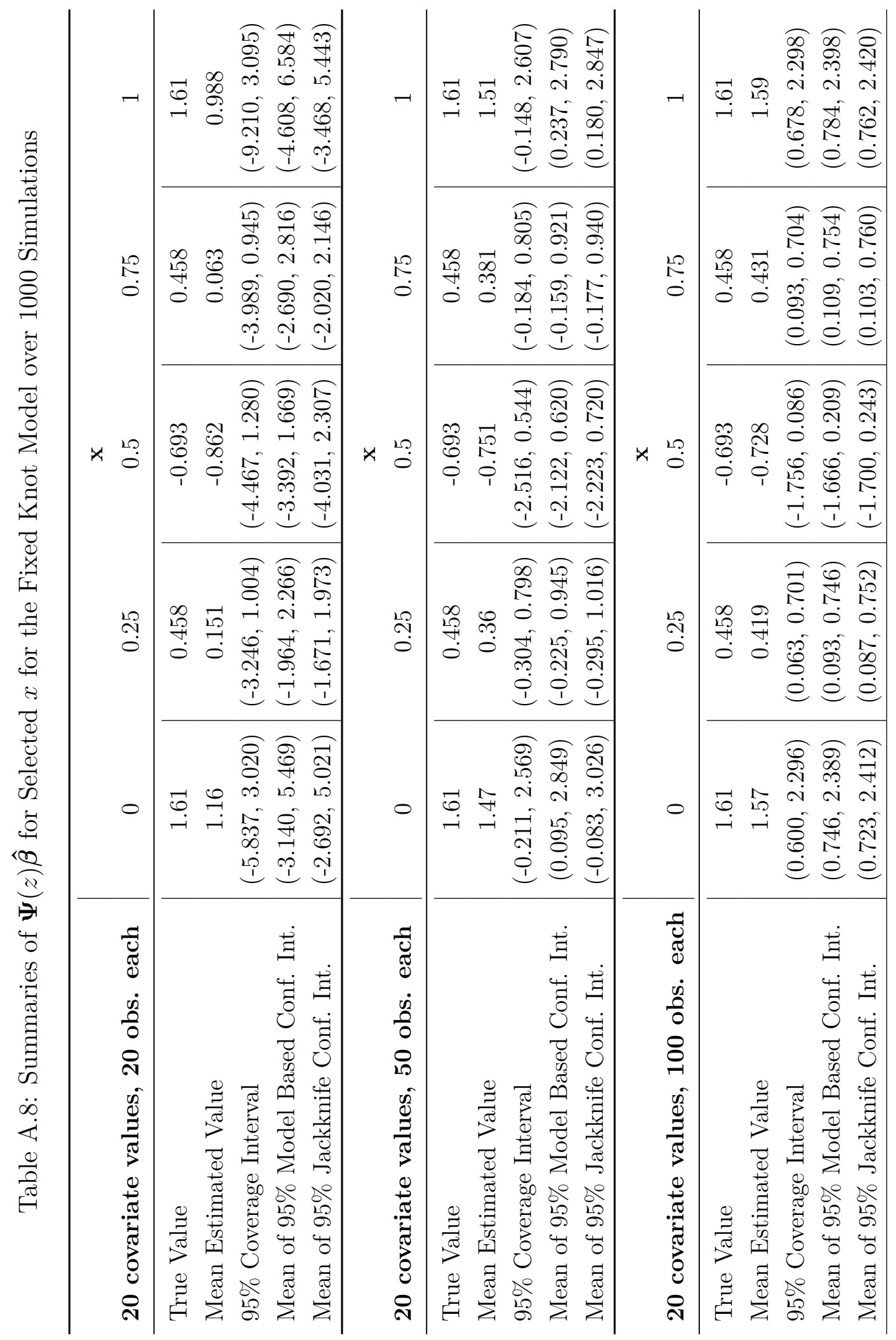




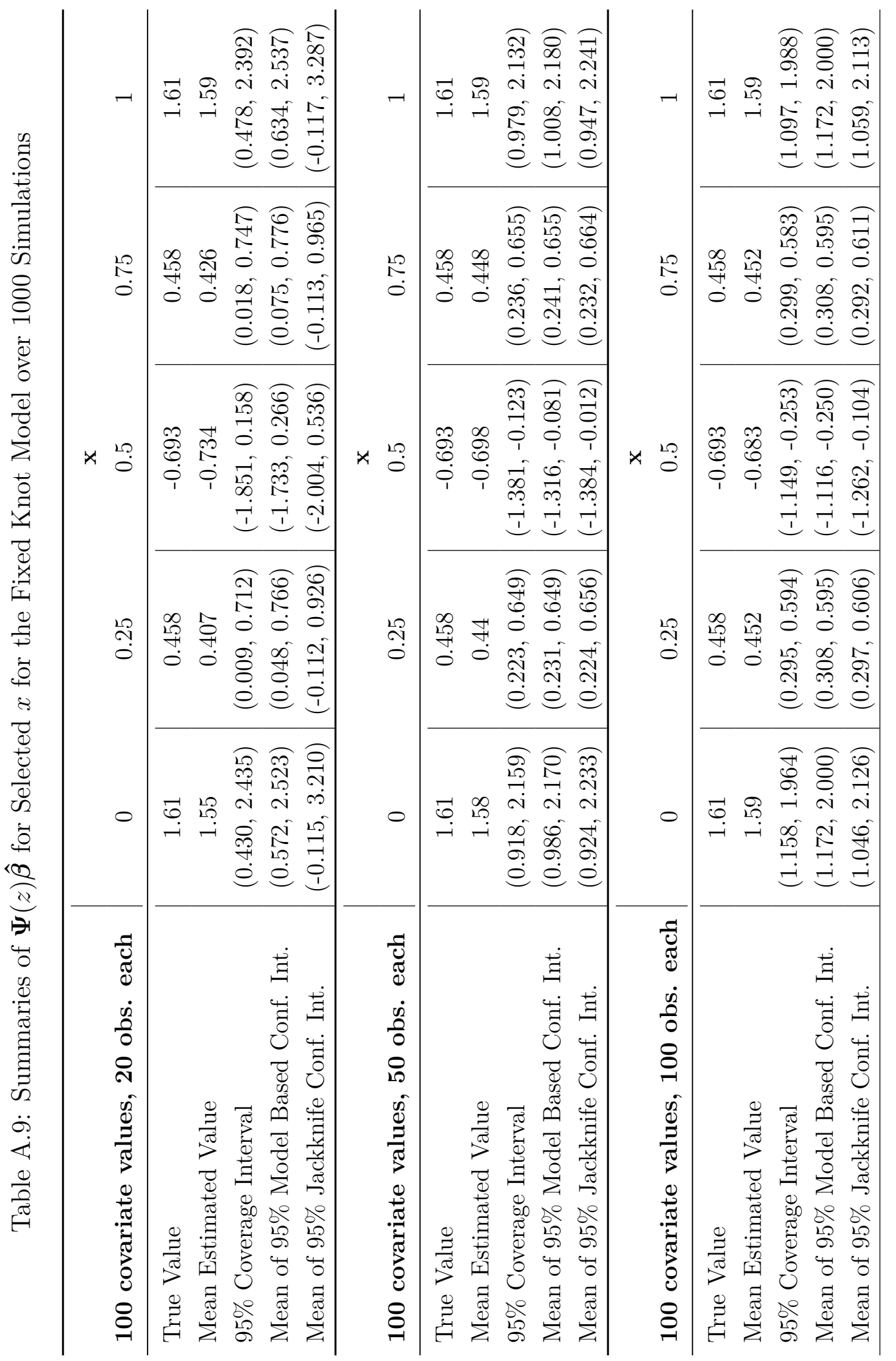




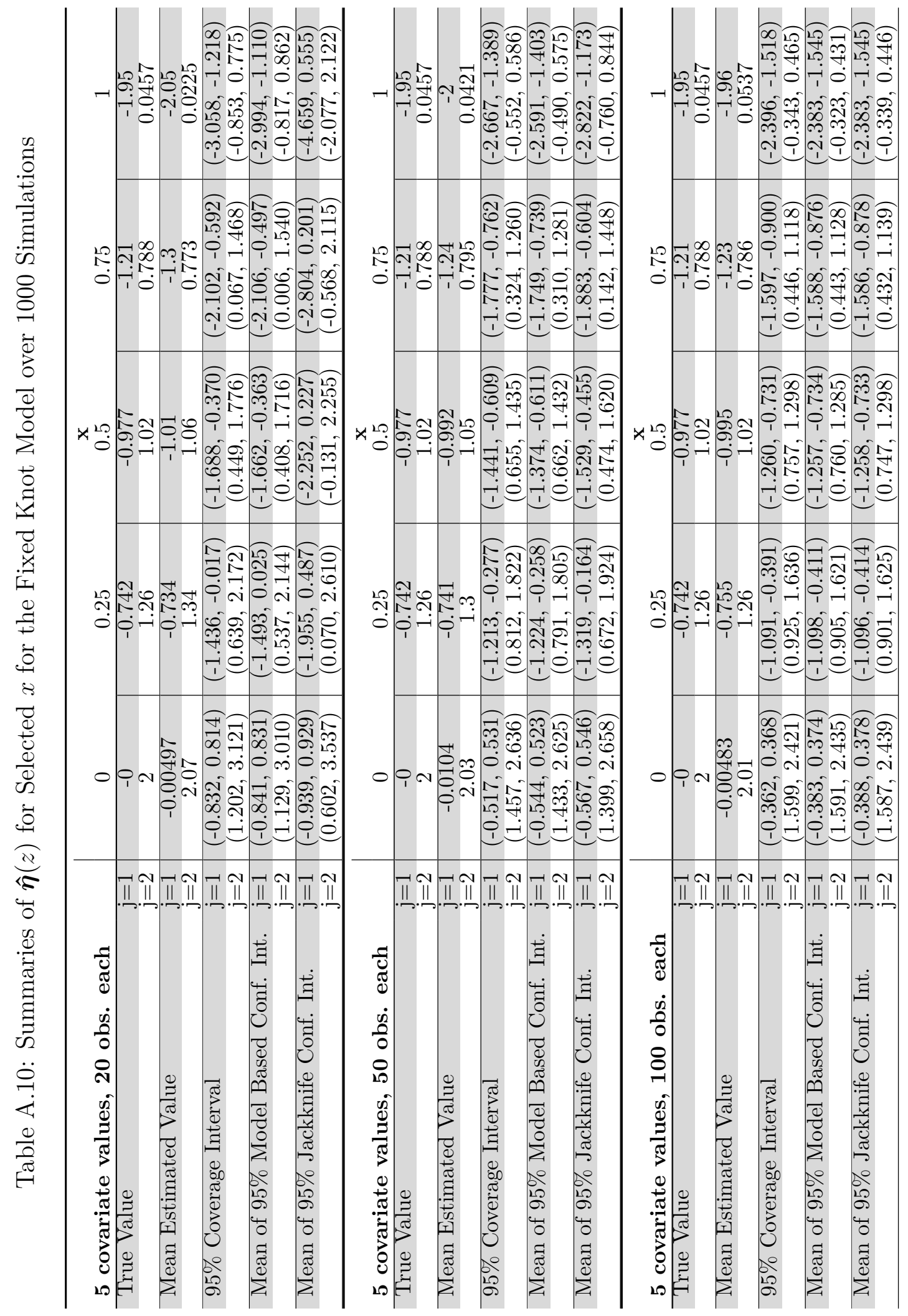




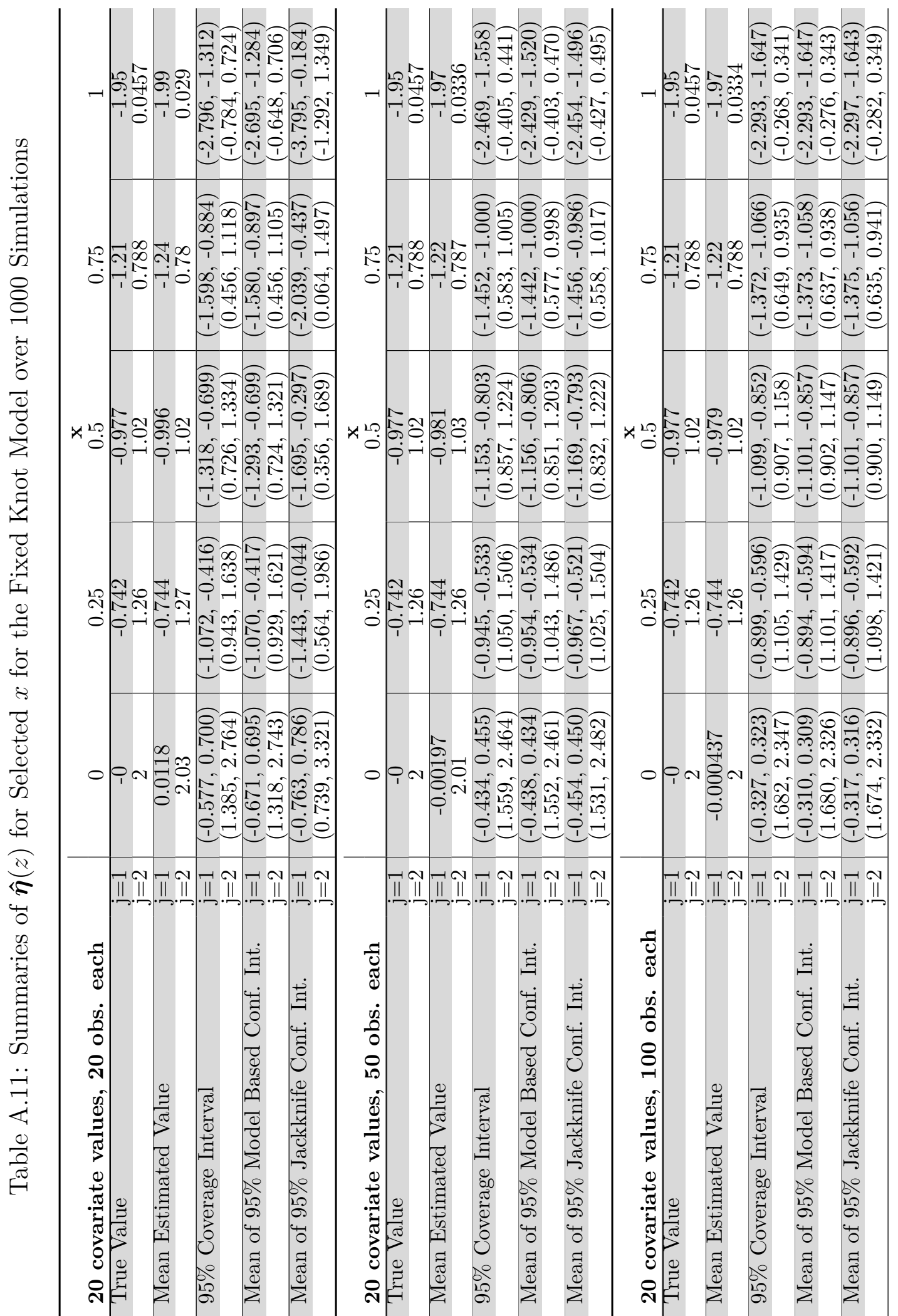




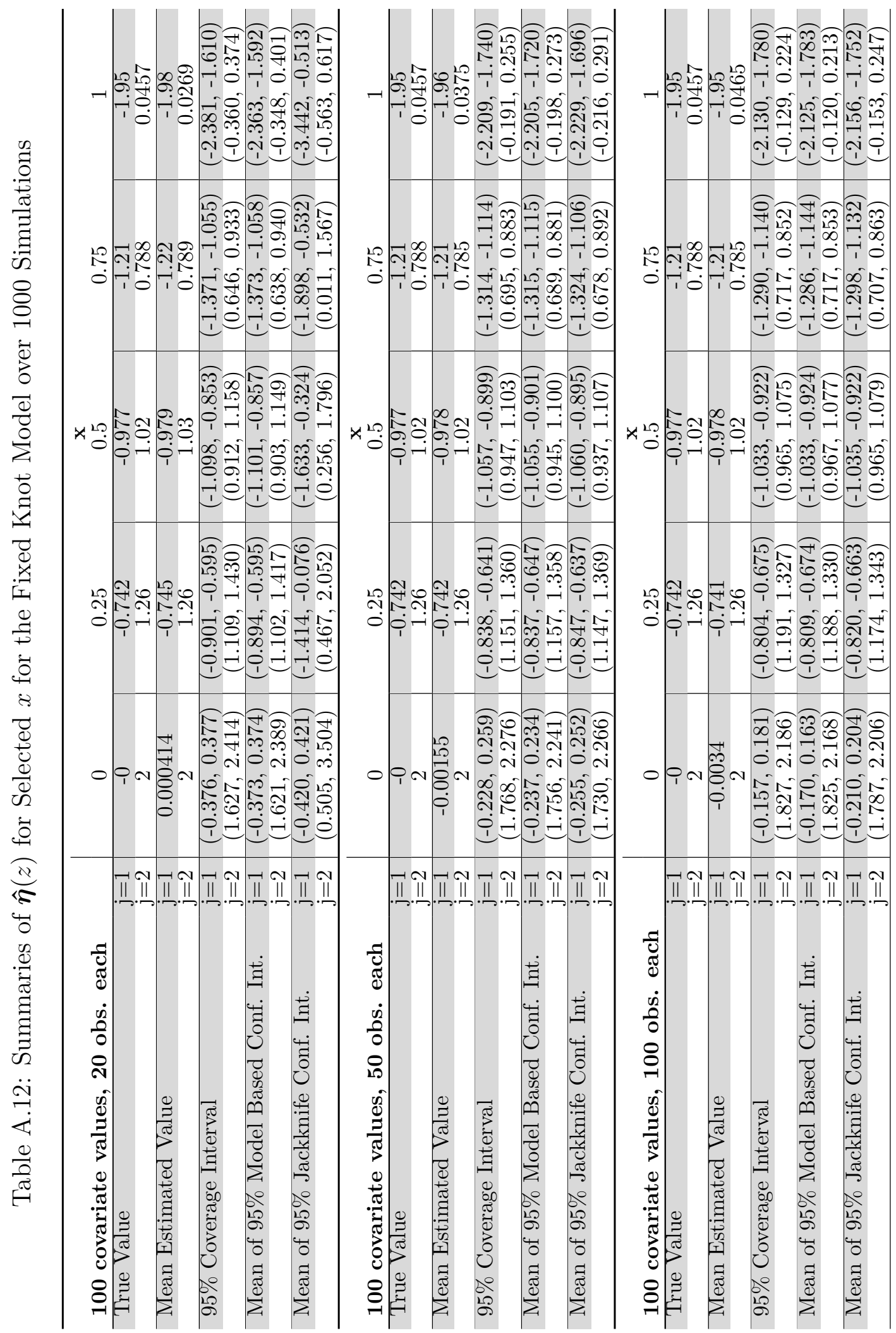









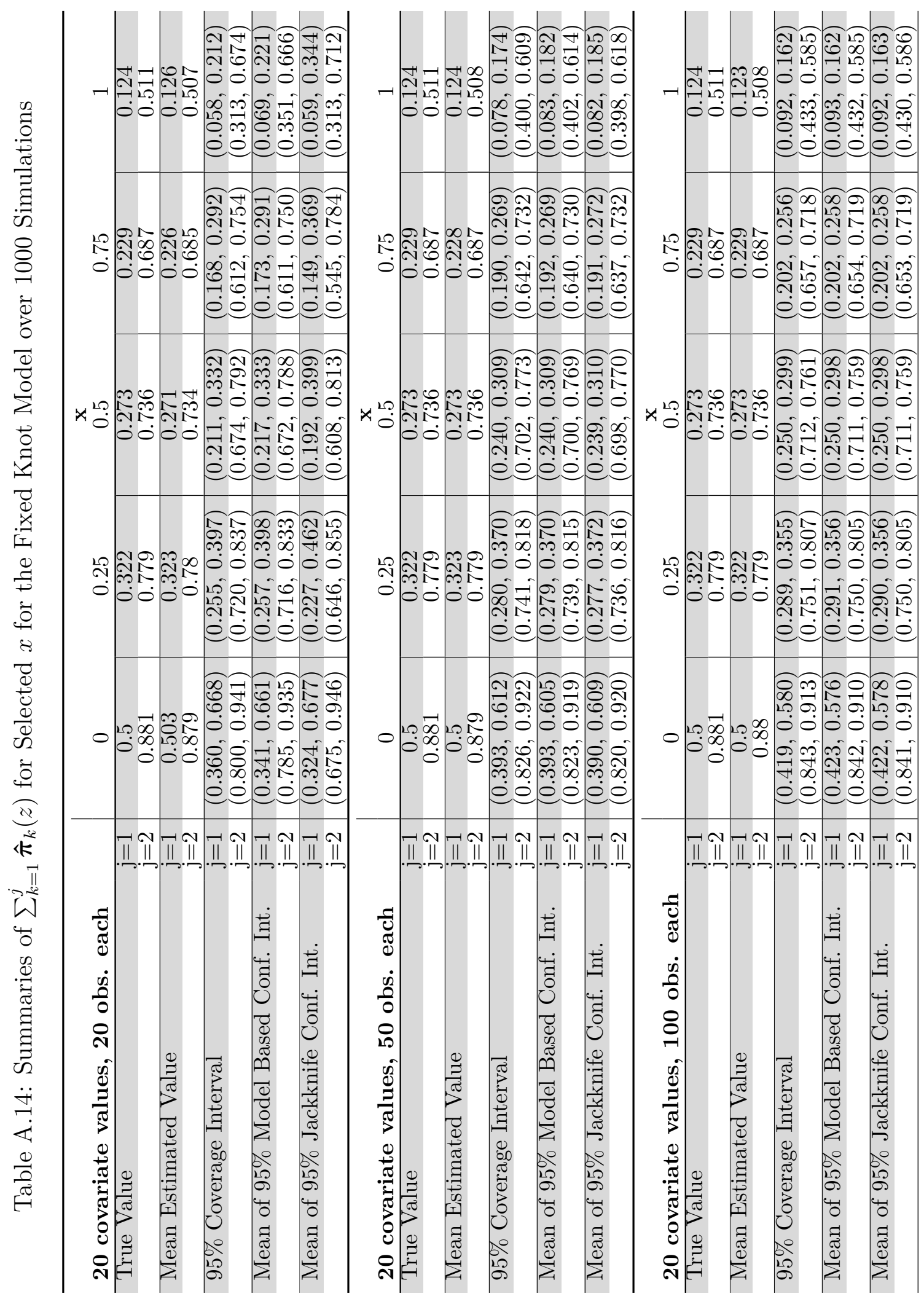




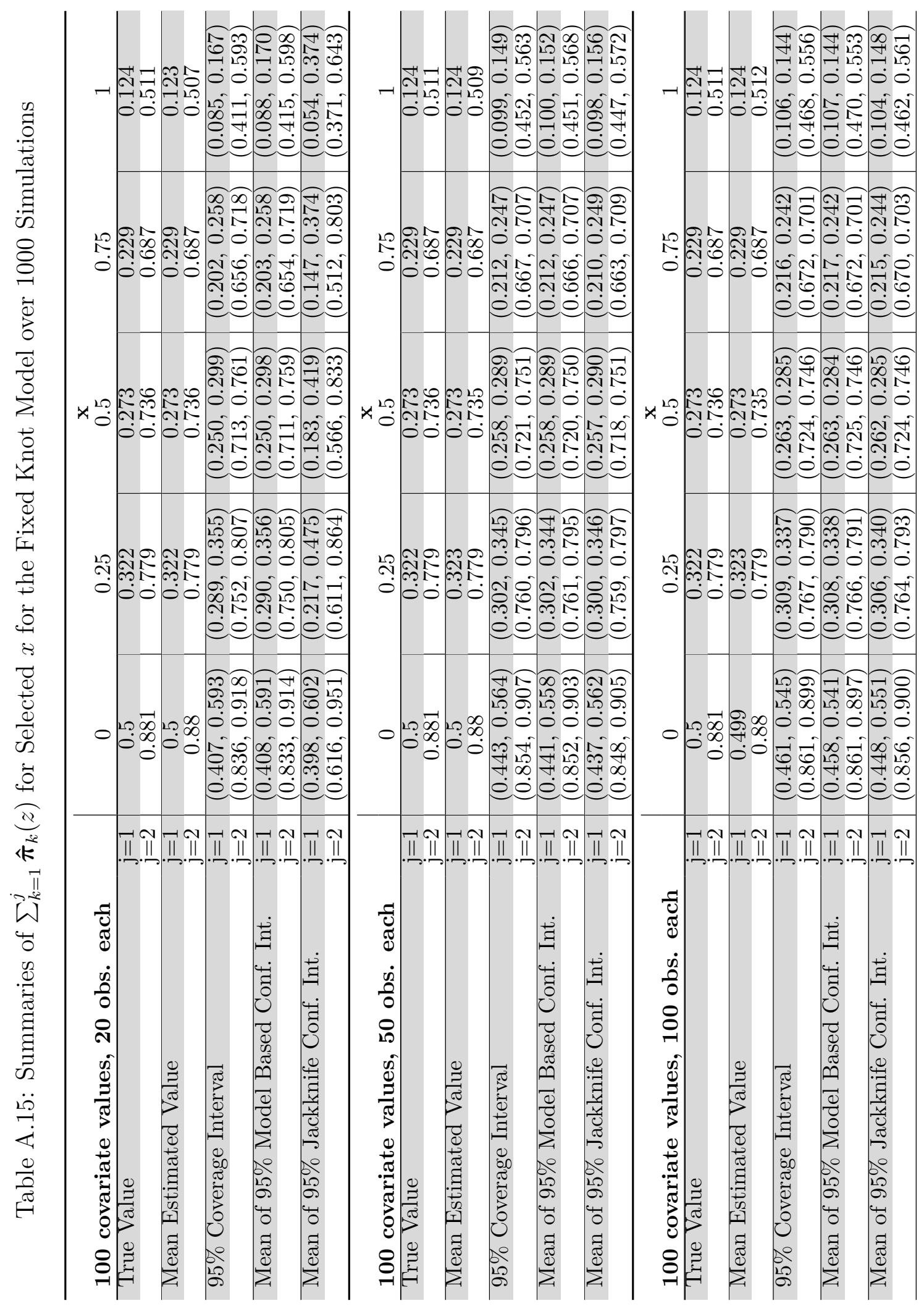




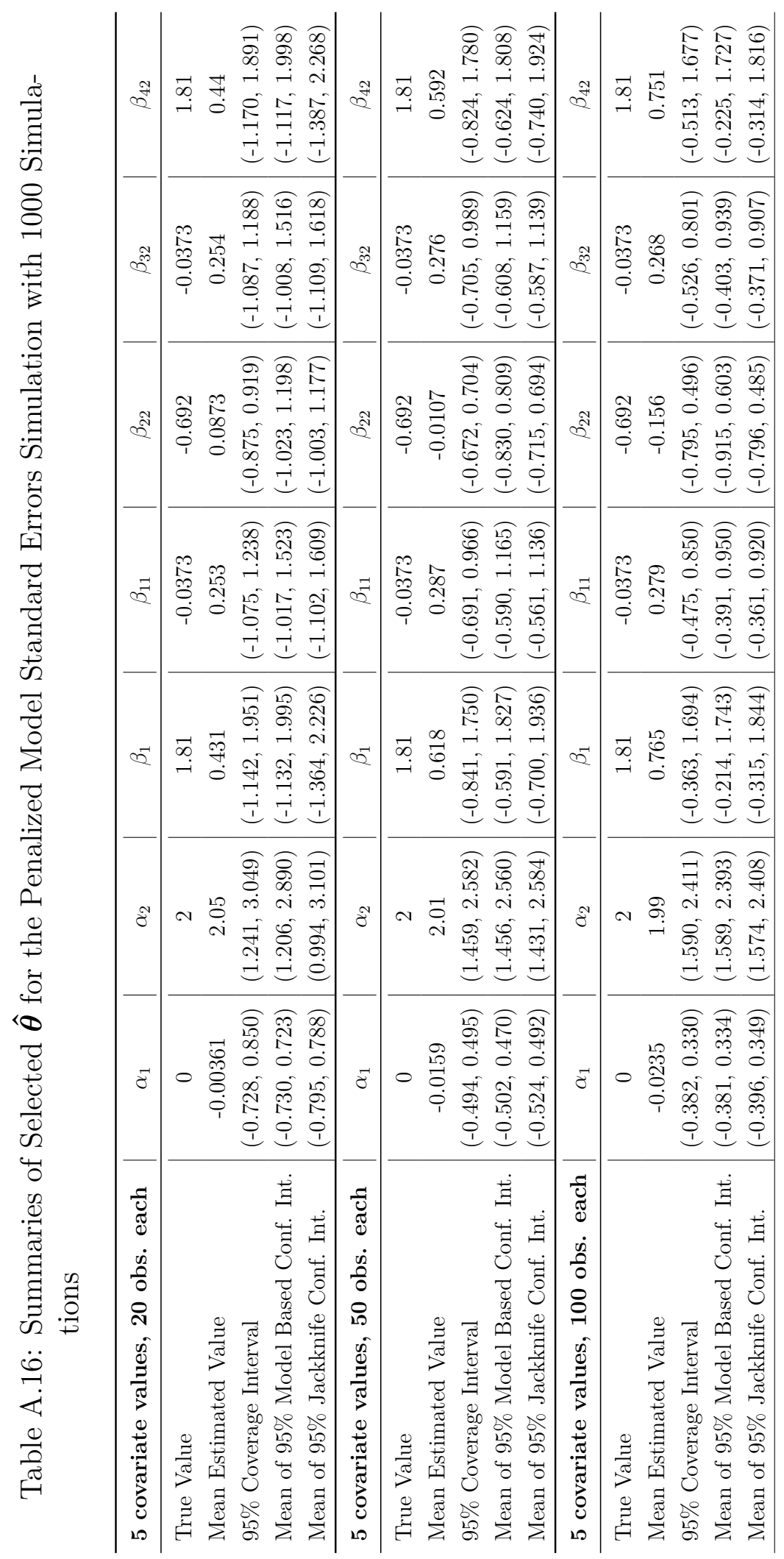




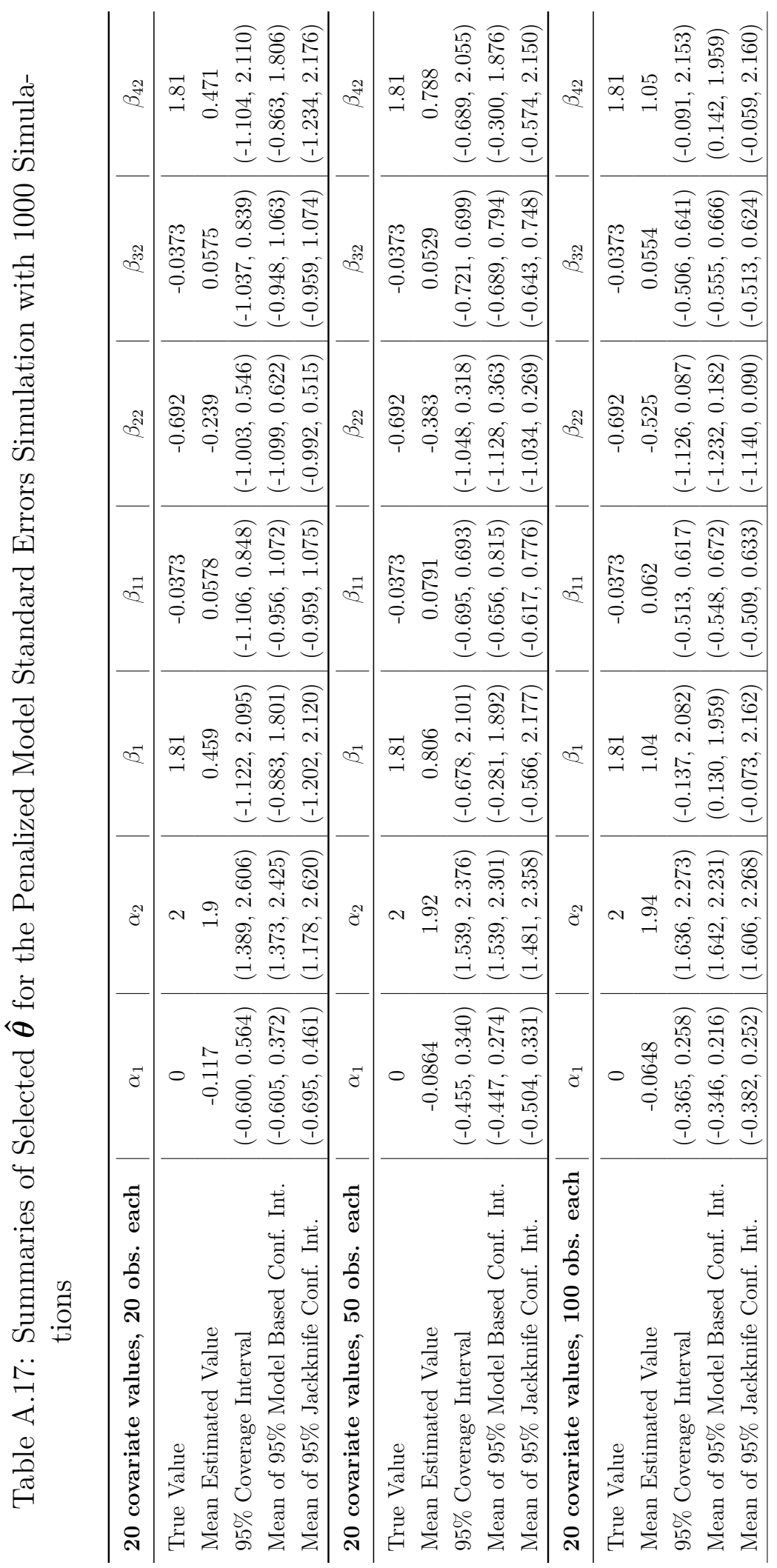




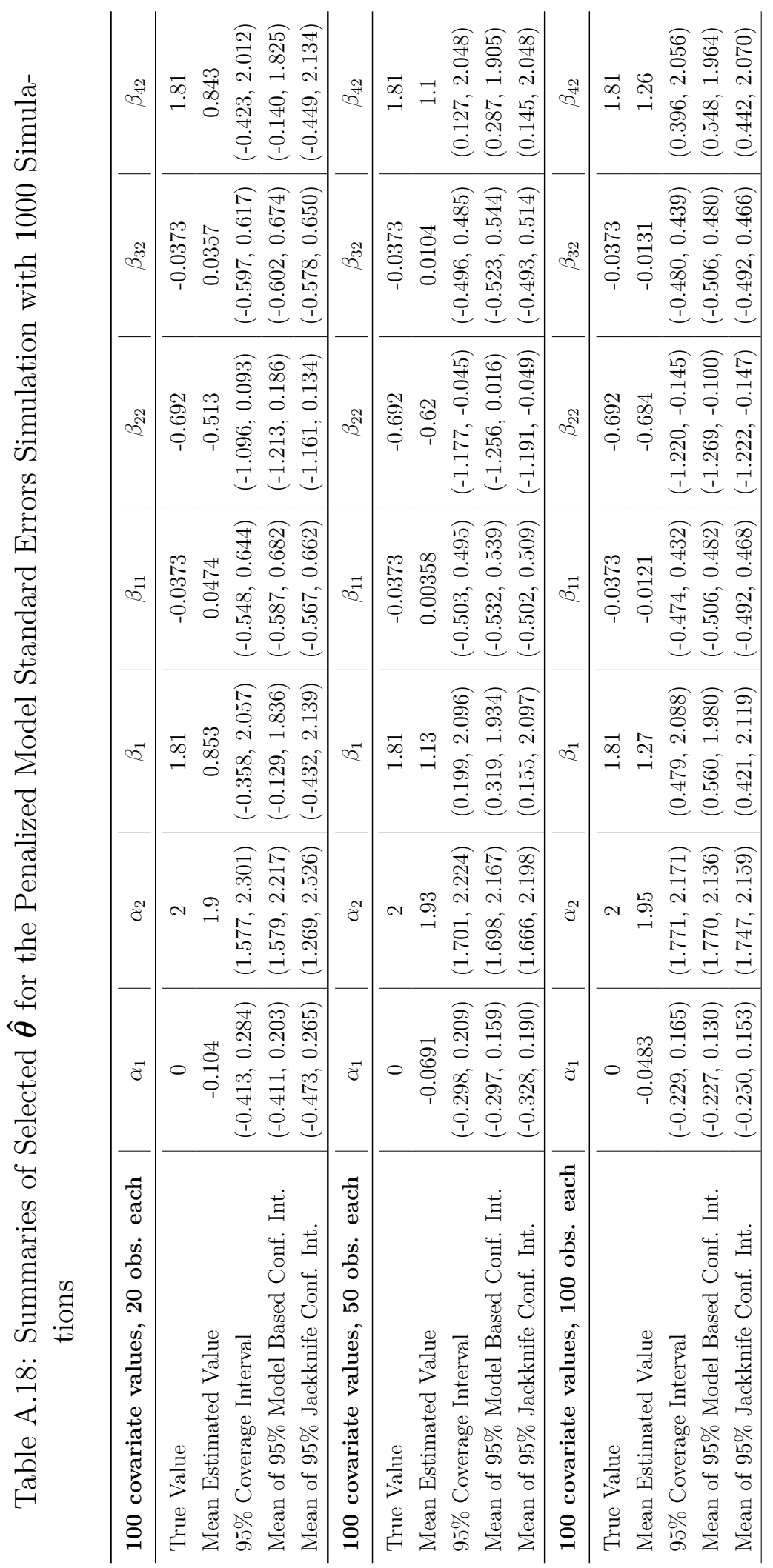




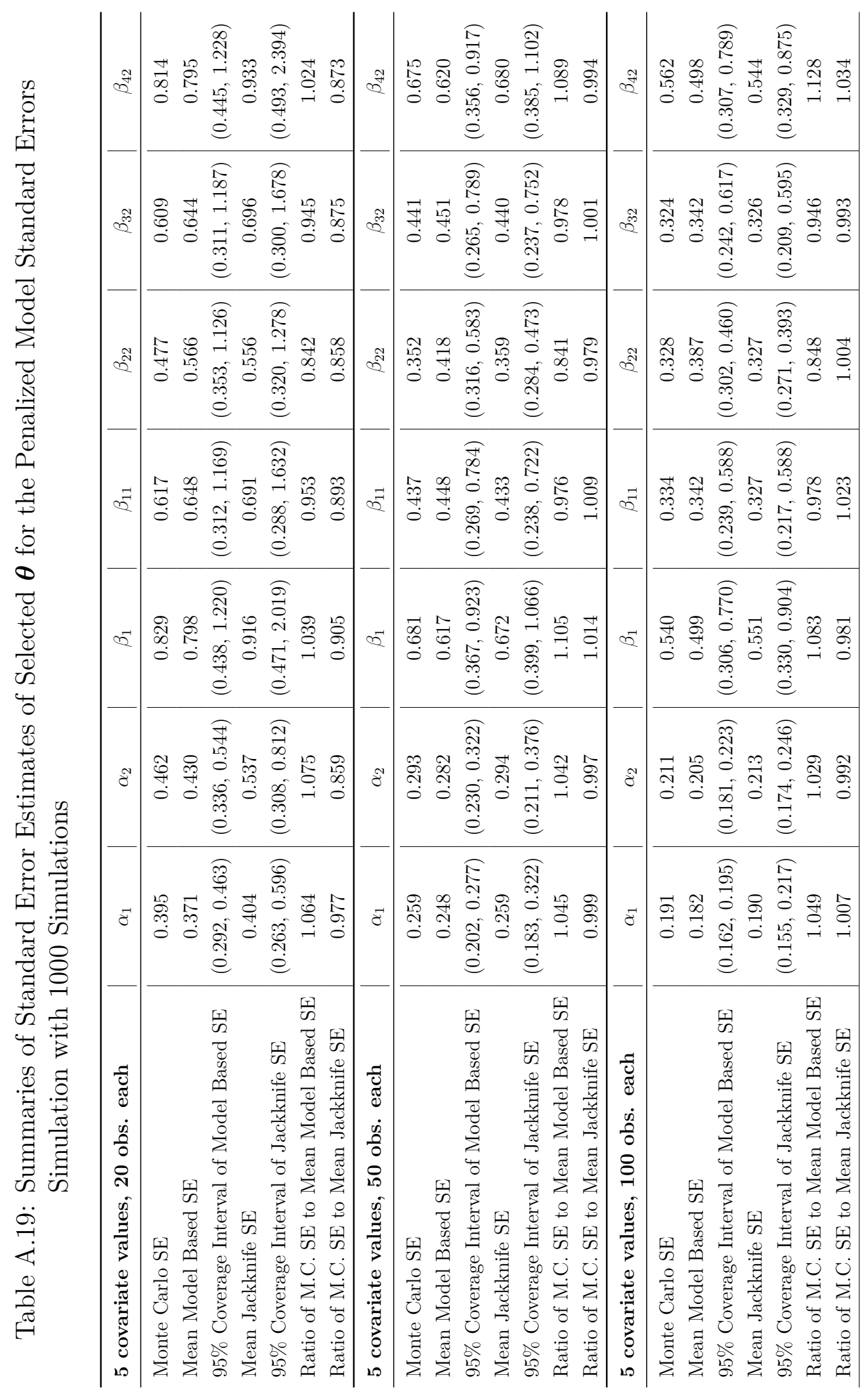




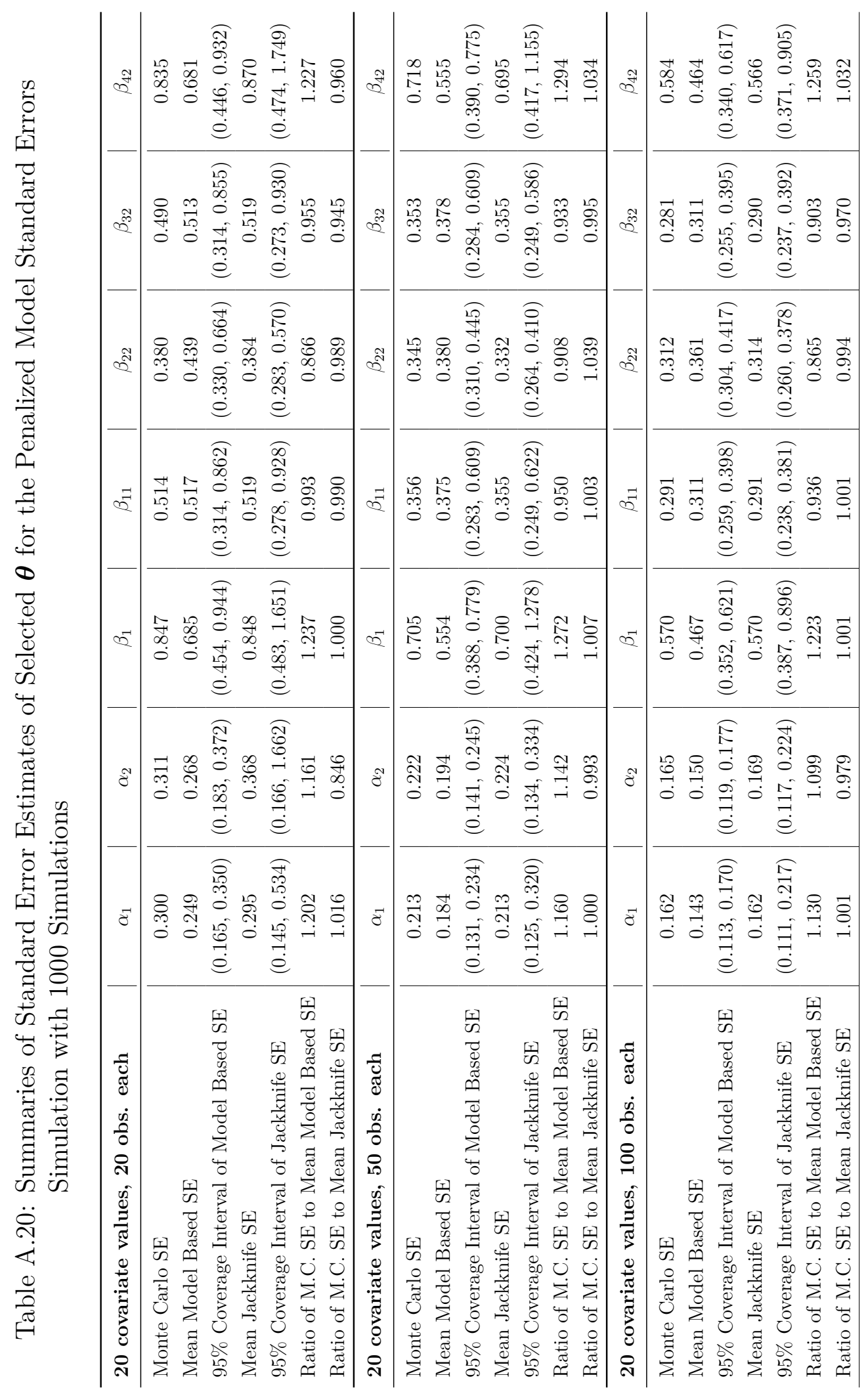




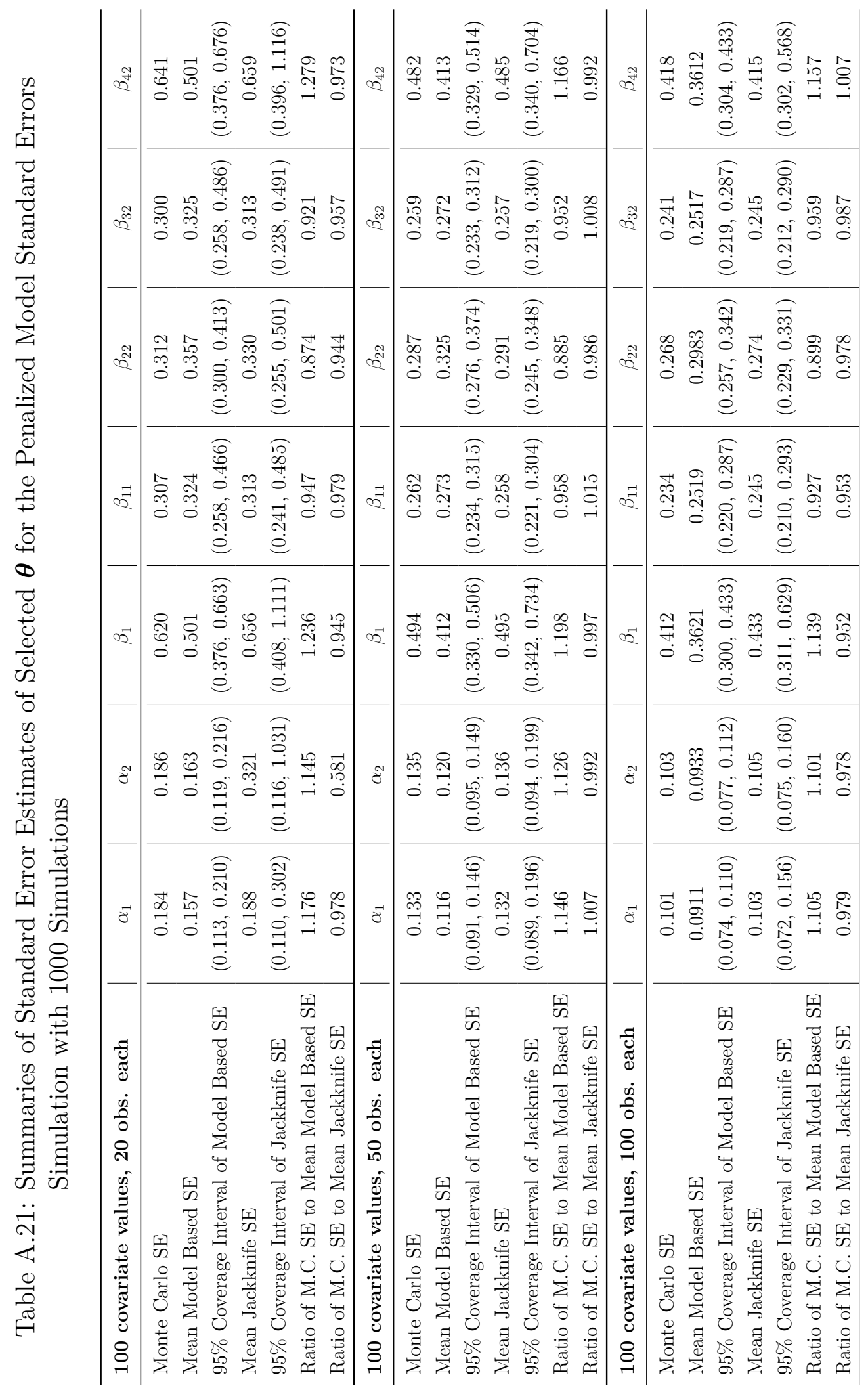




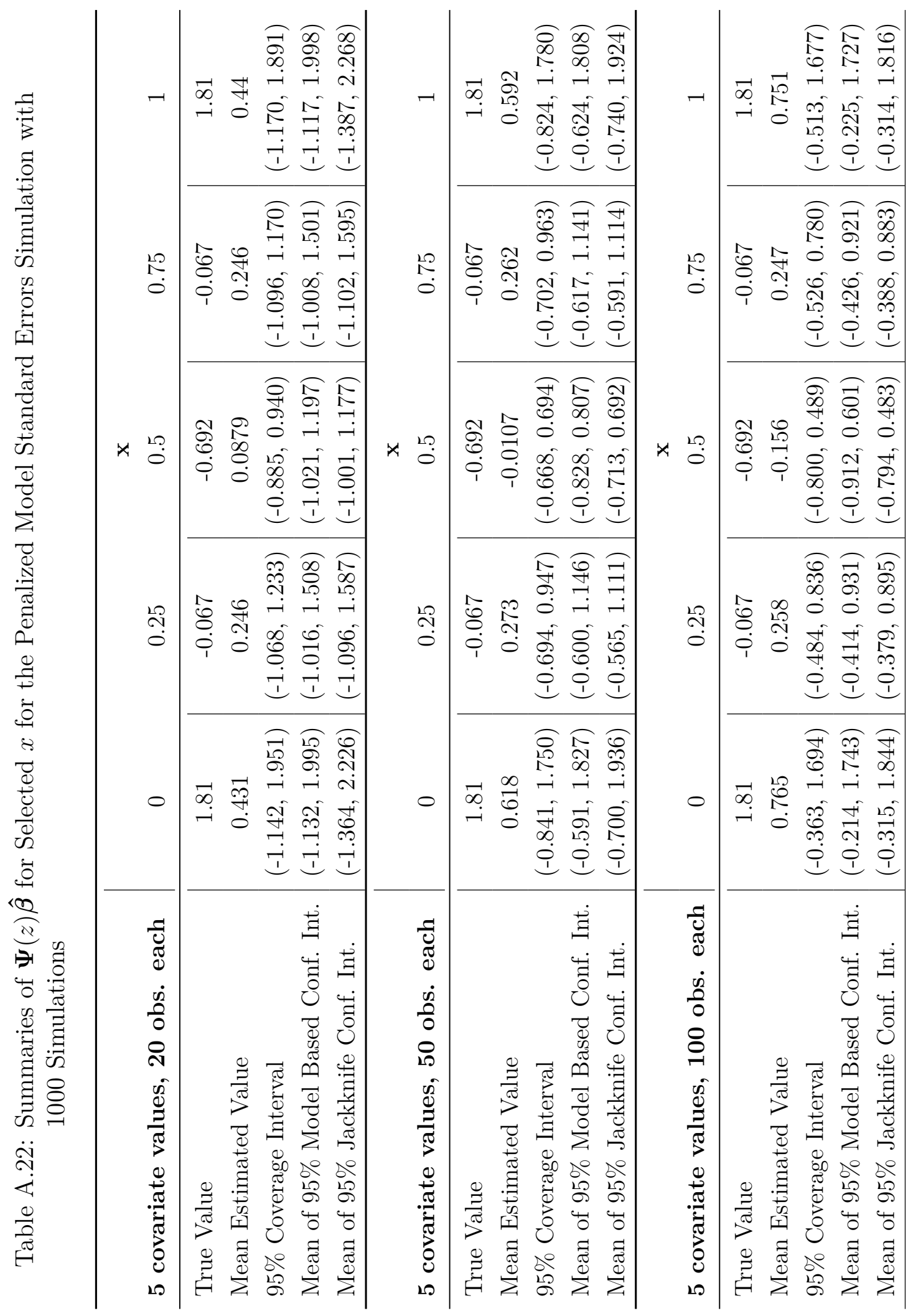




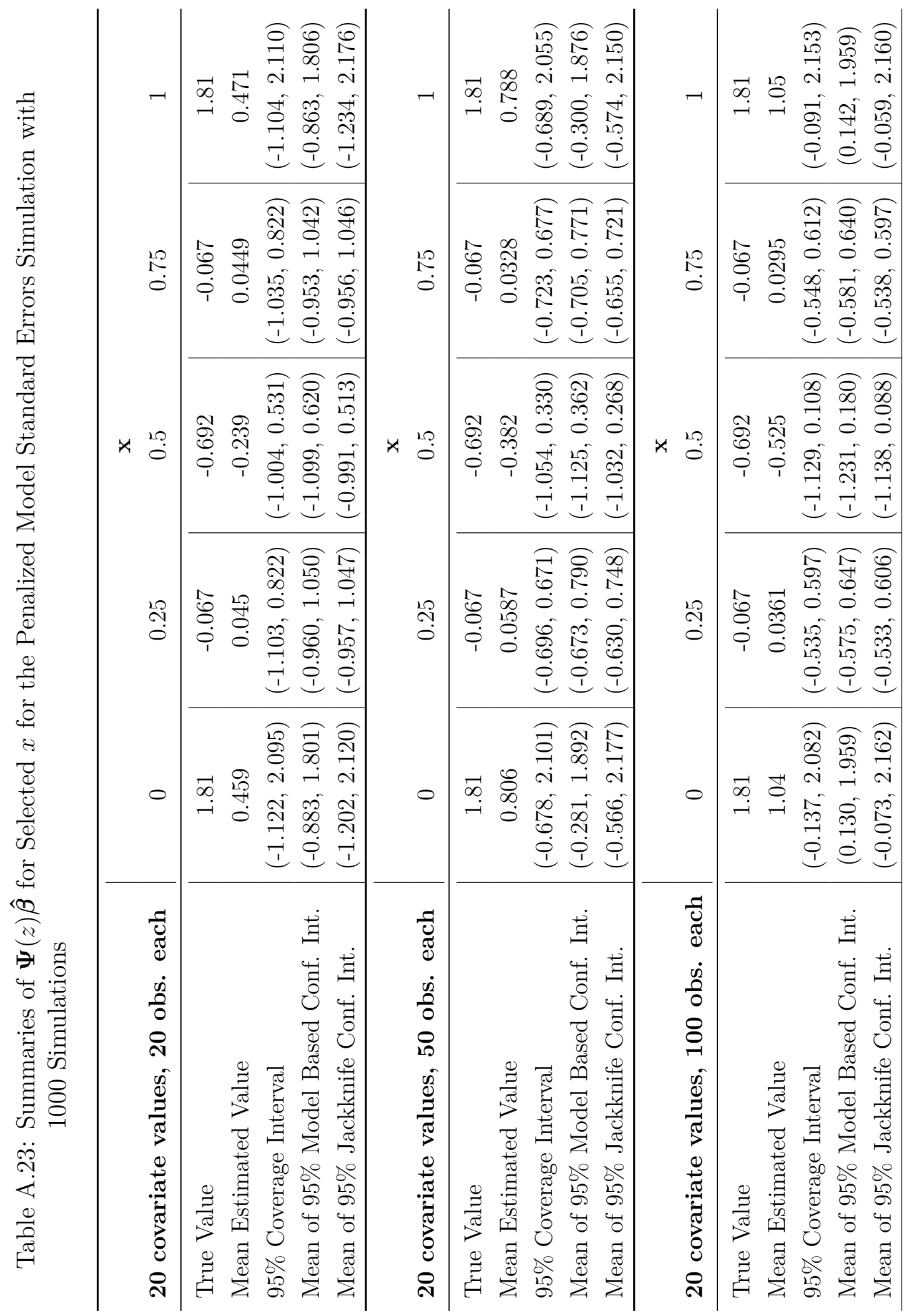




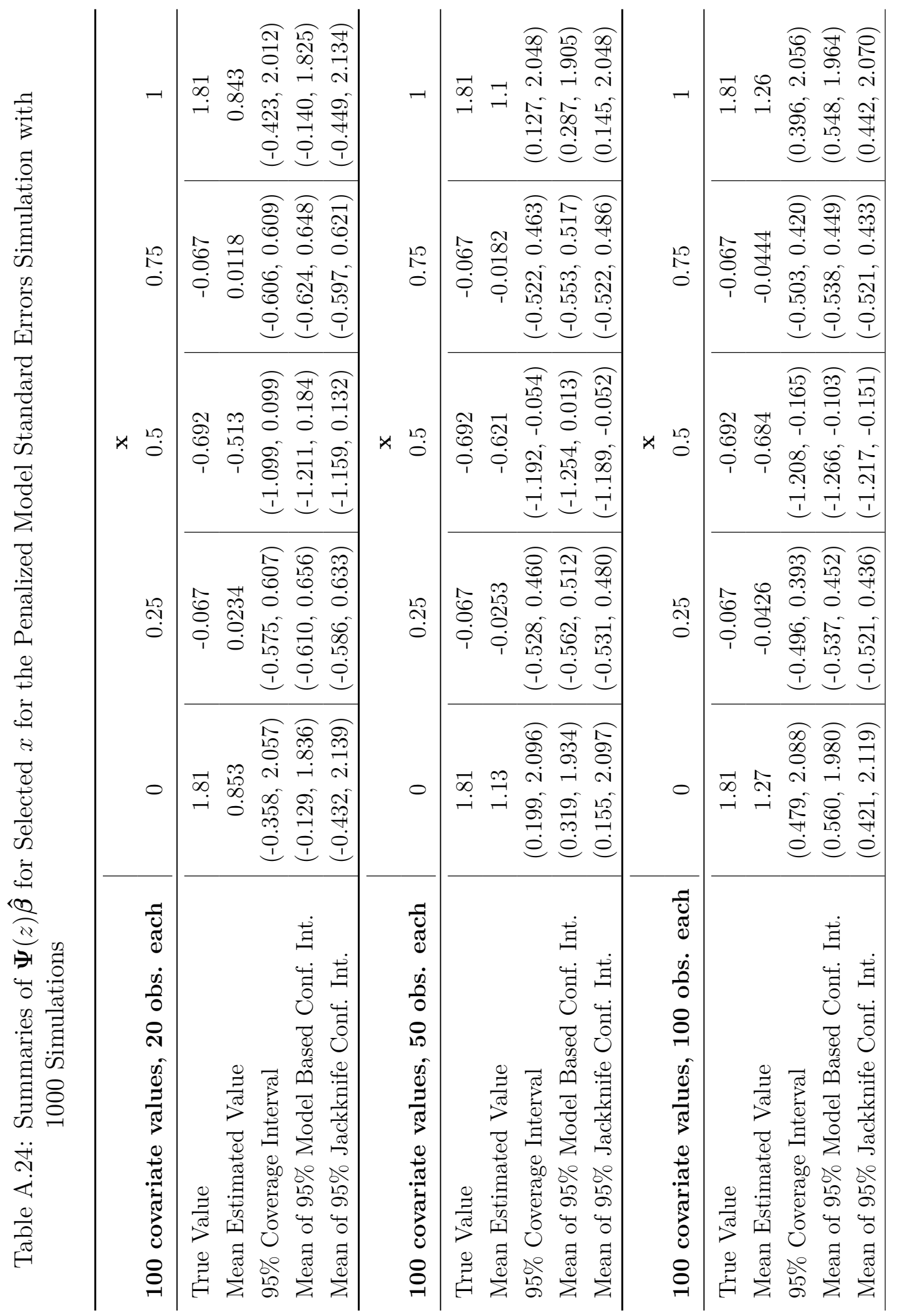




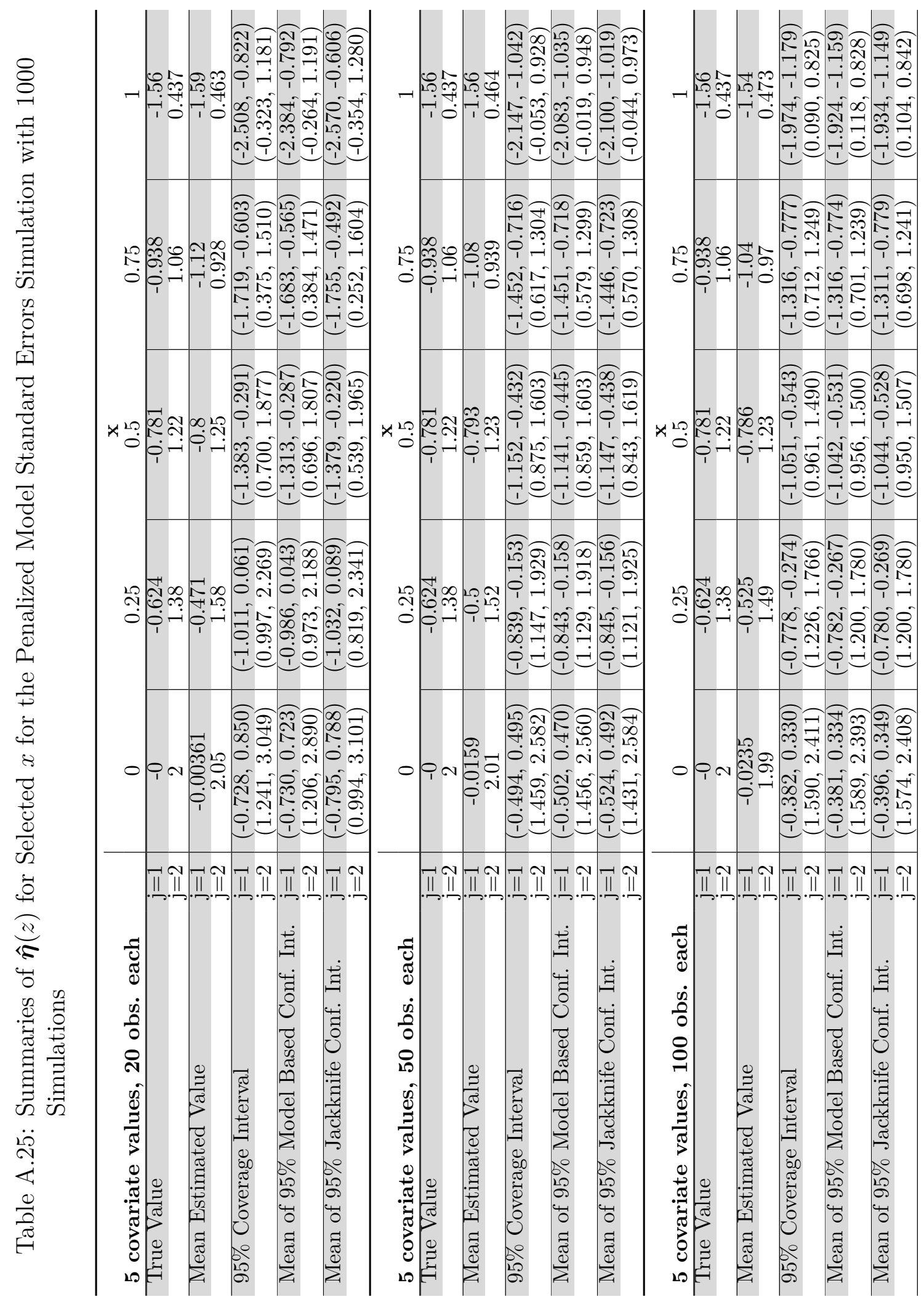




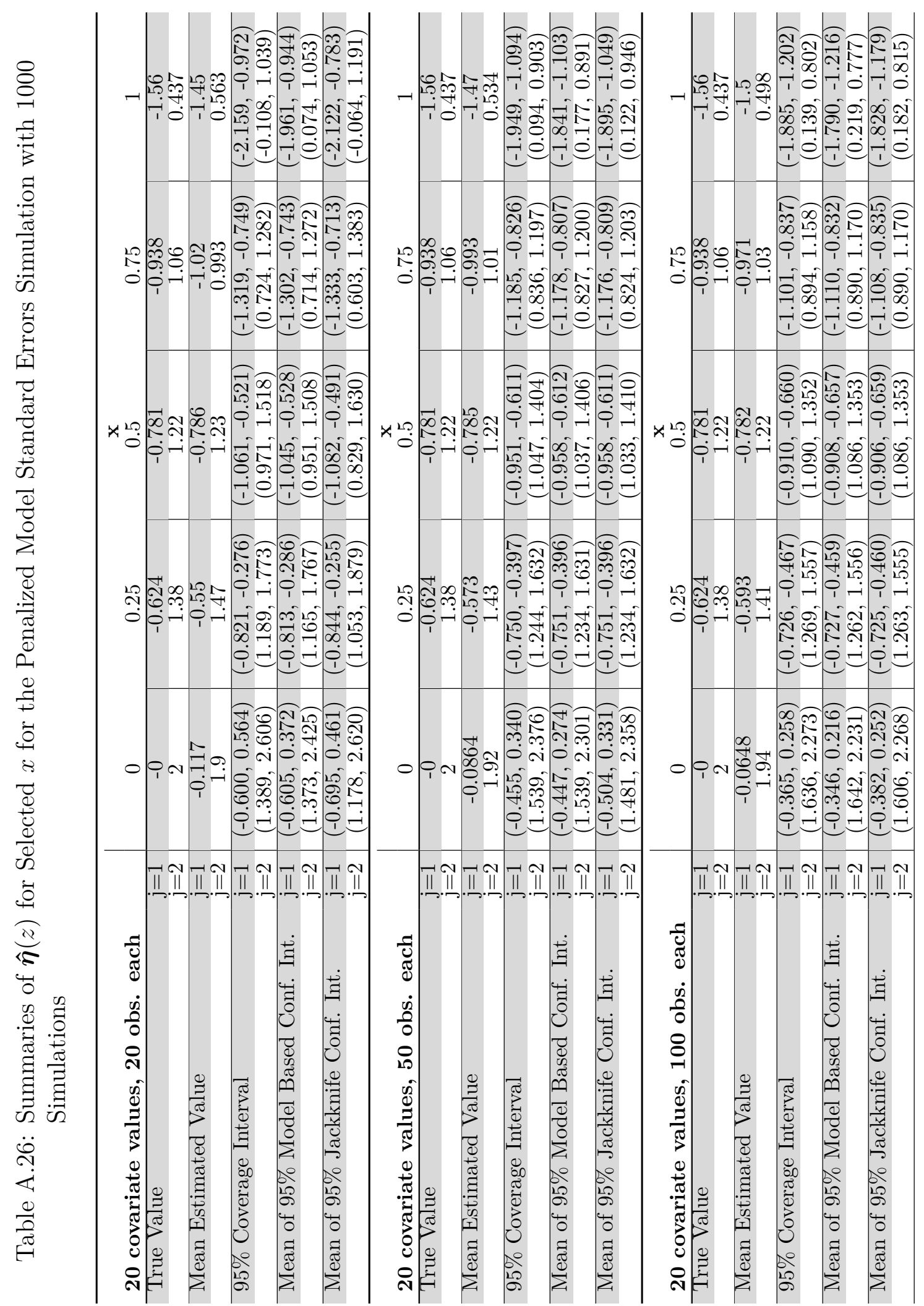




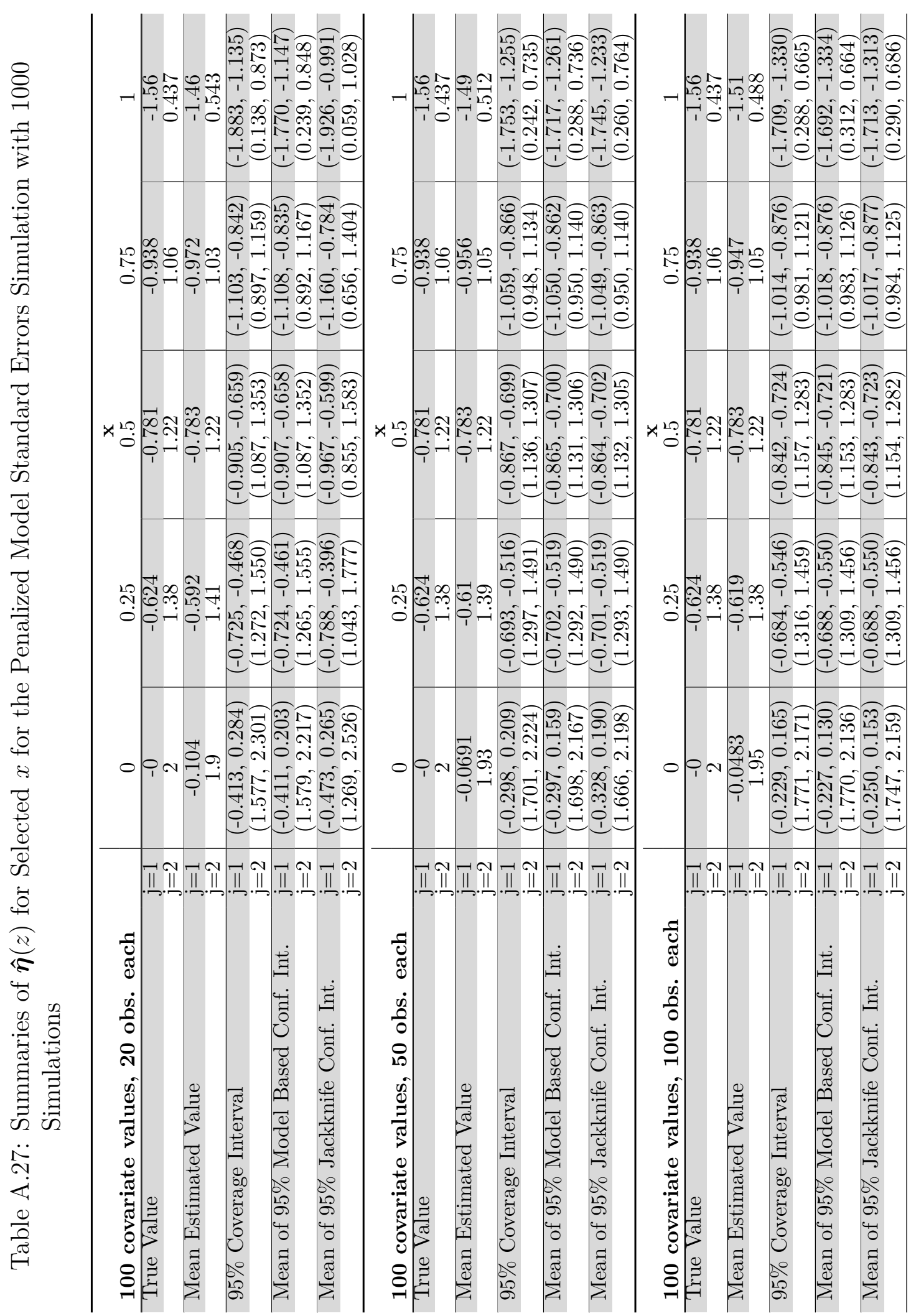




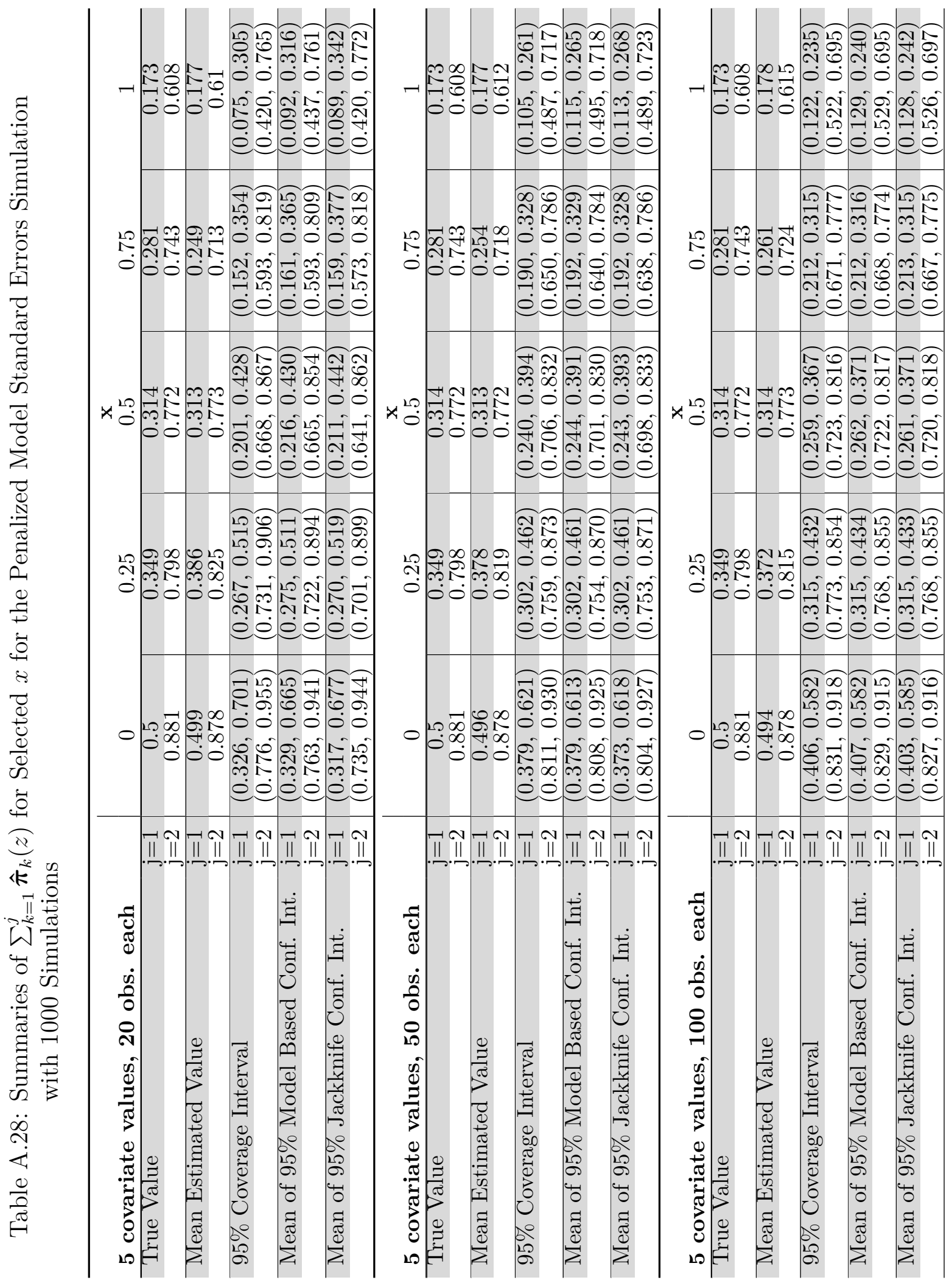




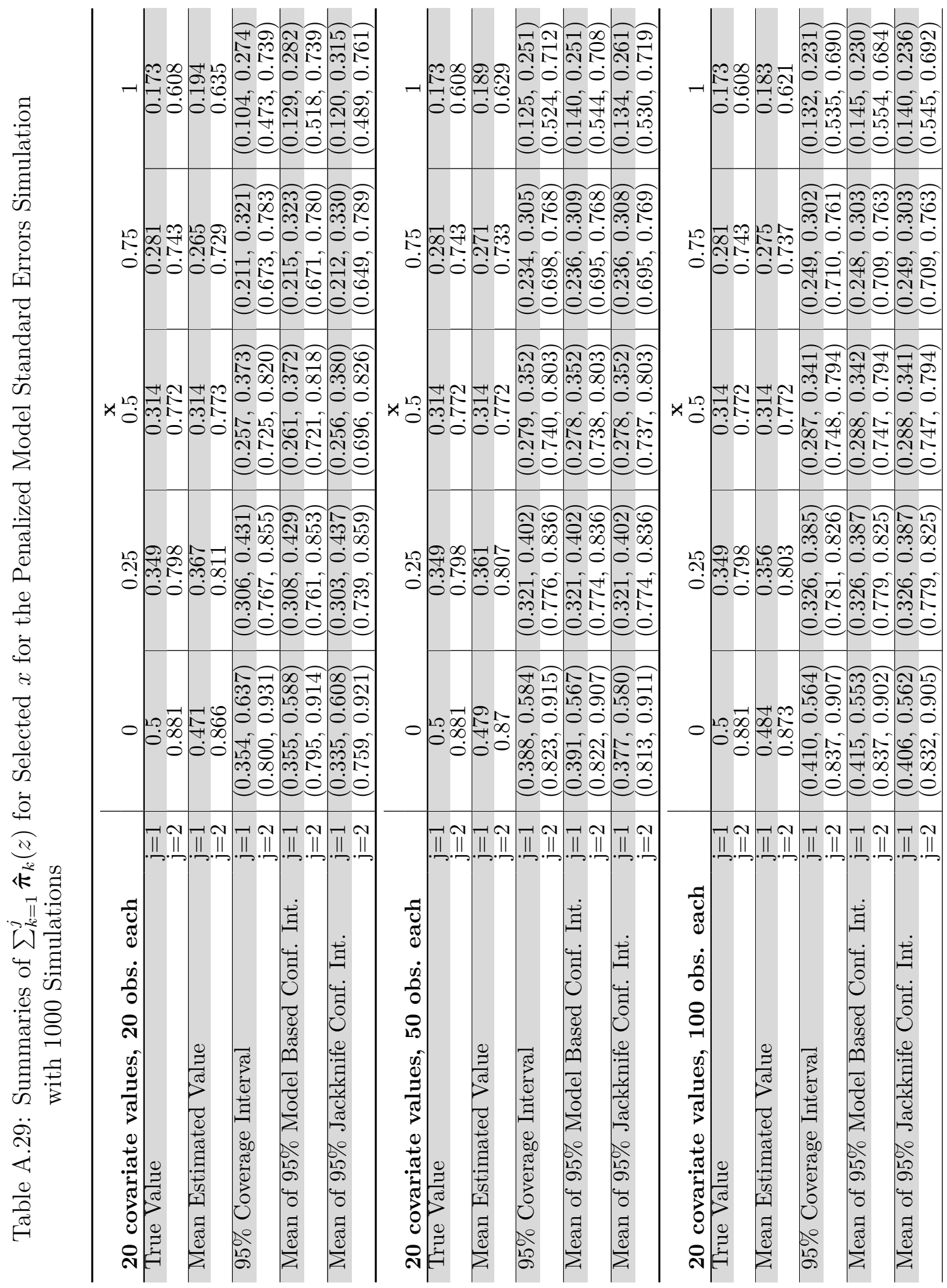







Appendix B

Benchmark Dose Simulation Results 


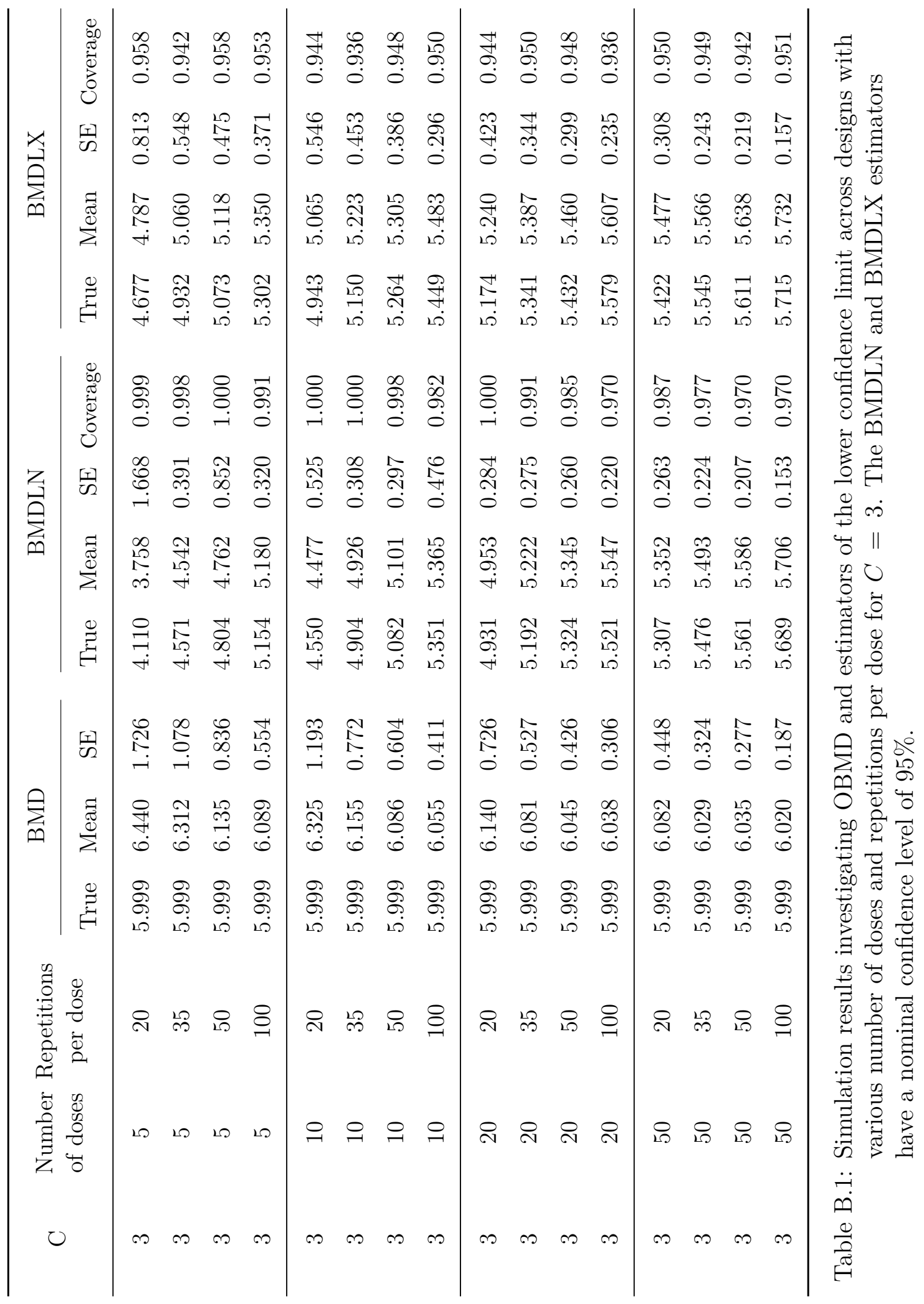









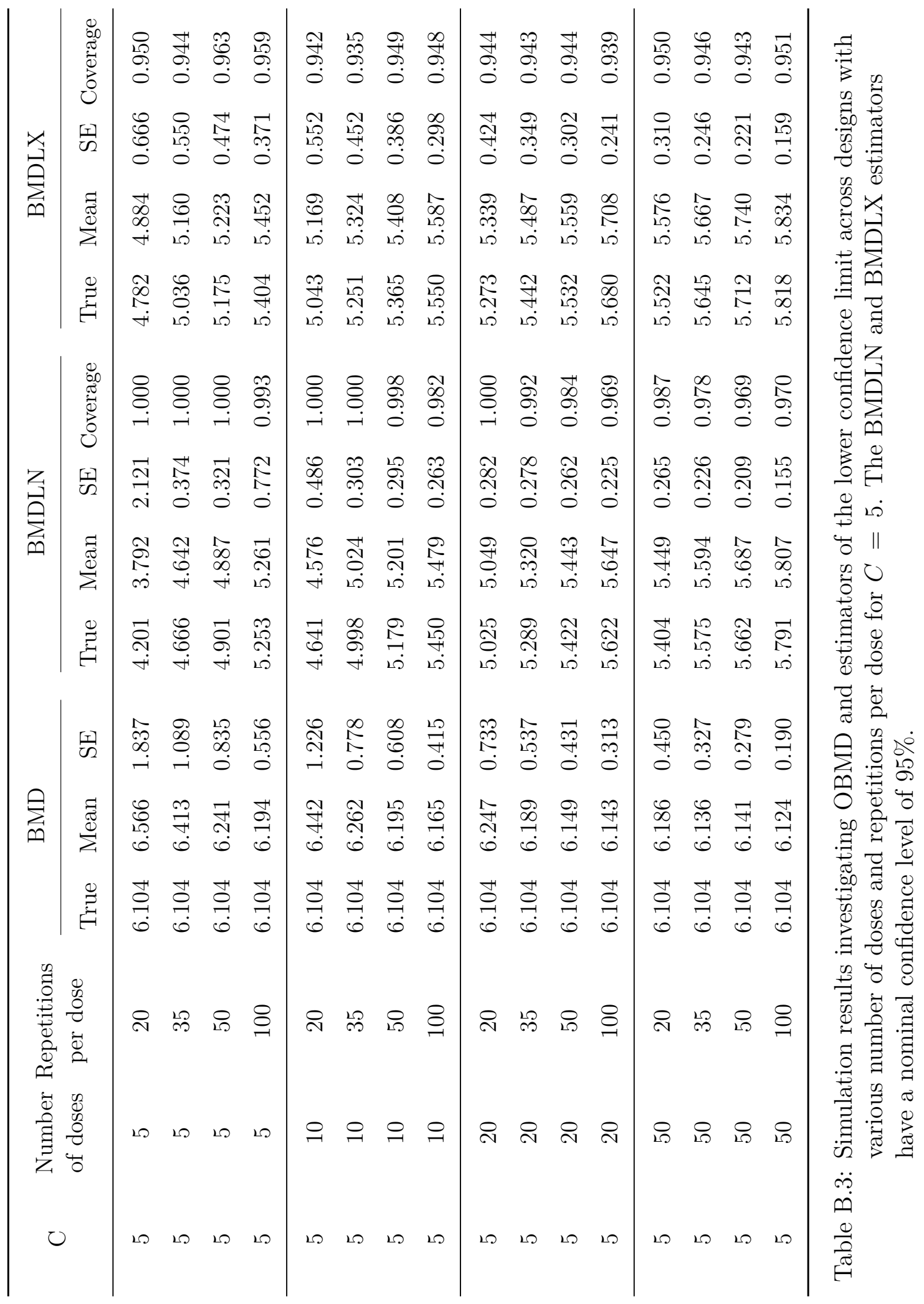

